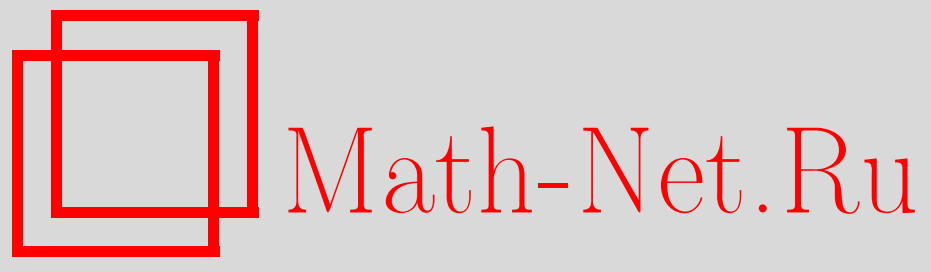

А. В. Маршаков, Матричные модели, комплексная геометрия и интегрируемые системы. I, ТМФ, 2006, том 147, номер 2, 163-228

DOI: https://doi.org/10.4213/tmf1959

Использование Общероссийского математического портала Math-Net.Ru подразумевает, что вы прочитали и согласны с пользовательским соглашением http://www . mathnet.ru/rus/agreement

Параметры загрузки:

IP : 44.207 .124 .84

26 апреля 2023 г., 16:56:55

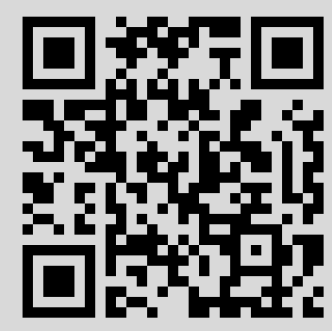




\title{
МАТРИЧНЫЕ МОДЕЛИ, КОМПЛЕКСНАЯ ГЕОМЕТРИЯ И ИНТЕГРИРУЕМЫЕ СИСТЕМЫ. І
}

\begin{abstract}
Рассматриваются простейшие калибровочные теории, представляемые одноматричными и двухматричными интегралами, особое внимание уделено их струнным и геометрическим свойствам. Описаны общие интегрируемые структуры, связанные с матричными интегралами, затем изучаются геометрические свойства матричных моделей в планарном пределе и демонстрируется, что существует их универсальная формулировка, непосредственно связанная с теорией комплексных кривых. Исследуются основные составляющие этой геометрической картины, которые формулируются в терминах квазиклассических интегрируемых систем, решаемых с помошью построения тау-функций или препотенциалов и предлагающих ее возможное обобщение на многомерный комплексный случай. Подробно обсуждаются комплексные кривые и тау-функции одноматричной и двухматричной моделей.
\end{abstract}

Ключевые слова: теория струн, матричные модели, комплексная геометрия.

\section{СОДЕРЖАНИЕ}

1. Калибровочные поля, геометрия и теория струн $\ldots \ldots \ldots \ldots \ldots \ldots \ldots \ldots \ldots . \ldots \ldots 4$

2. Матричные модели и интегрируемость ......................... 168

2.1. Матричные ансамбли и цепочка Тоды ........................... 168

2.2. Матричные интегралы в переменных Мивы ................. 172

2.3. Интегралы Концевича, свободные фермионы и топологические

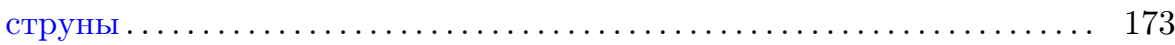

3. Планарный предел одноматричной модели . . . . . . . . . . . . . . . . . 175

3.1. Интеграл по собственным значениям и комплексная кривая ........ 175

3.2. Свободная энергия: геометрическое определение .............. 177

Статья написана по заказу Редколлегии по материалам нескольких лекций, прочитанных на школах по математической физике, а также докладов на конференции "Комплексная геометрия и теория струн" и семинаре памяти М. К. Поливанова.

* Физический институт им. П. Н. Лебедева РАН, Институт теоретической и экспериментальной физики, Москва, Россия. E-mail: mars@lpi.ru, mars@itep.ru 
4. Квазиклассическая тау-функция . . . . . . . . . . . . . . . . . . . 181

4.1. Римановы поверхности, голоморфные и мероморфные дифференци-

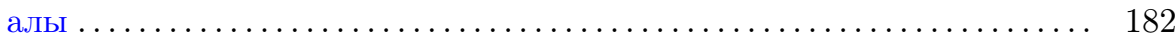

4.2. Определение квазиклассической тау-функции ................ 184

4.3. Препотенциал в многомерном случае .................... 187

4.4. Тау-функция одноматричной модели $\ldots \ldots \ldots \ldots \ldots \ldots \ldots \ldots \ldots \ldots . \ldots \ldots$

4.5. Формула вычетов .................................. 191

4.5.1. Голоморфные дифференциалы . . . . . . . . . . . . . . . . 191

4.5.2. Мероморфные дифференциалы ....................... 193

5. Комплексная кривая двухматричной модели...................... 195

5.1. Квазиклассическое приближение для двухматричной модели ....... 195

5.2. Уравнение и род кривой в комплексной задаче ................. 197

5.3. Структура комплексной кривой двухматричной модели ............. 201

5.4. Вырождения кривой максимального рода................... 203

6. Двухматричная модель и задача Дирихле ....................... 206

6.1. Задача Дирихле и интегрируемость....................... 206

6.2. Многосвязный случай и дубль Шоттки .................... 212

6.3. Квазиклассическая тау-функция многосвязной области ............ 218

6.4. Полиномиальные потенциалы и алгебраические области . . . . . . . . 220

6.5. Преобразование дуальности и свободная энергия двухматричной мо-

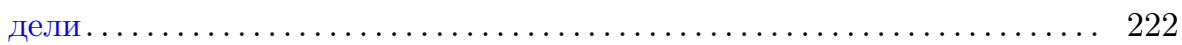

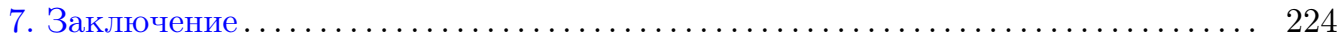

Приложение 1. Рациональные вырождения ...................... 224

Приложение 2. Координаты в многосвязном случае ................ 225

\section{1. КАЛИБРОВОЧНЫЕ ПОЛЯ, ГЕОМЕТРИЯ И ТЕОРИЯ СТРУН}

Связь между калибровочными теориями и теорией струн до сих пор является одной из наиболее интересных проблем современной теоретической физики (см., например, [1]). На сегодняшний день ее понимание продвинулось далеко за пределы хорошо известного факта, что спектр открытых струн на плоском фоне содержит векторное поле, связанное с простейшим струнным возбуждением, а репараметризационная инвариантность квантовой механики струнных мод требует безмассовости этого возбуждения.

Изучение пространства модулей теории замкнутых струн указывает на естественность появления и в этом случае открытых струн, концы которых расположены лишь на определенных гиперповерхностях или $D$-бранах [2]. При этом пачка из $N$ $D$-бран приводит к появлению матричного, или неабелева калибровочного поля, у которого матричные индексы нумеруют браны, на которых расположены концы соответствующей струны. Эта картина позволяет сформулировать калибровочную теорию с группой $S U(N)$ на геометрическом языке, и таким образом мы приходим к естественному выводу, что калибровочные поля или пачка $D$-бран изменяют геометрию фоновых полей в теории замкнутых струн (подробное обсуждение этих 
вопросов см., например, в [3]-[5]). Это позволяет предположить, что калибровочная теория может быть переформулирована на языке некоторой теории гравитации, возможно, в пространстве-времени с бо́льшим числом измерений.

В неабелевой калибровочной теории запрещены цветные состояния (гипотеза конфайнмента). На языке замкнутой теории струн это означает, что модификация фоновых полей замкнутой струны должна приводить к тому, что состояния открытых струн исчезают из спектра. На языке статистической суммы (или производящей функции для струнных амплитуд) это условие можно записать в виде равенства

$$
Z_{\text {gauge }}=e^{F_{\text {string }}}
$$

где $Z_{\text {gauge }}$ представляет собой статсумму некоторой калибровочной теории, в то время как соответствующая свободная энергия $F_{\text {string }}$ является функцией распределения уже некоторой теории замкнутых струн.

Простейшей (но не единственной!) геометрической интерпретацией соотношения (1.1) является $1 / N$-разложение [6] по обратному размеру матриц со стороны калибровочной теории. Теория возмущения в квантовой теории поля имеет нулевой радиус сходимости, если суммировать по всем возможным диаграммам. Однако в матричной теории с ленточными фейнмановскими графиками каждая фейнмановская диаграмма может быть однозначно ассоциирована с двумерной поверхностью фиксированной топологии, на которой она может быть изображена без самопересечений. Таким образом, возникает дополнительный параметр - род графа или соответствующей римановой поверхности, и разложение по графам заданной топологии уже может быть просуммировано в пределах некоторого конечного радиуса сходимости.

Это означает, что в любой матричной теории с матрицами размера $N \times N$ возникает двойное разложение

$$
F_{\text {string }}=\sum_{g=0}^{\infty} N^{2-2 g} \sum_{h} F_{g, h} \curlywedge^{h} \text {, }
$$

где $g$ обозначает введенный род римановой поверхности, отвечающей фейнмановскому графу, у которого $V$ вершин, $E$ ребер и $F$ граней, с эйлеровой характеристикой $\chi_{g}=V-E+F=2-2 g$. Дополнительная сумма по "дыркам" $h=2 g-2+F$ или граням графов, нарисованных на заданной римановой поверхности, может быть интерпретирована как суммирование (с производящим параметром ^, который мы определим ниже) по всем вкладам открытых струн, которые, будучи просуммированными, исчезают из спектра теории, меняя геометрию замкнутых струн.

Модельным примером калибровочной теории, который будет обсуждаться ниже, является нуль-мерная теория или матричный интеграл (впервые обсуждавшийся в данном контексте в работах [7])

$$
Z=\int d \Phi e^{-\operatorname{Tr} W(\Phi) / \hbar}
$$


где $\Phi$ - матрица размера $N \times N$ (например, эрмитова), а $W(\Phi)$ - некоторый полином общего вида

$$
W(\Phi)=\sum_{k>0} t_{k} \Phi^{k} .
$$

Для матриц конечного размера $N \times N$ эта статсумма представляет собой просто $N^{2}$-кратный интеграл, который, тем не менее, обладает практически всеми известными нетривиальными свойствами континуальных интегралов квантовой теории поля. Очевидно, что действие $\operatorname{Tr} W(\Phi)$ в $(1.3)$ инвариантно относительно нуль-мерных калибровочных преобразований (где отсутствует член с производной) $\Phi \rightarrow U^{\dagger} \Phi U$. Мерой интегрирования в формуле (1.3) является определенным образом нормированное произведение дифференциалов всех матричных элементов

$$
d \Phi=\frac{1}{V_{N}} \prod d \Phi_{i j}
$$

где, как в любой калибровочной теории, нормировочный фактор $V_{N}$ содержит объем орбиты унитарной группы $U(N) / U(1)^{N}$ вместе с фактором $N$ !, ответственным за перестановки собственных значений. Такая нормировка становится естественной после разделения физических и калибровочных степеней свободы путем диагонализации матрицы $\Phi$ унитарным калибровочным преобразованием $\Phi=U^{\dagger} \phi U$, где $\phi$ - диагональная матрица. Объем орбиты $U(N) / U(1)^{N}$ затем сокращается после тривиального интегрирования по калибровочным степеням свободы.

Рассмотрев в качестве примера потенциал

$$
W(\Phi)=\frac{1}{2} \Phi^{2}+\frac{g_{\mathrm{YM}}}{3} \Phi^{3}+\cdots,
$$

где по аналогии с теорией Янга-Миллса мы обозначили константу связи $g_{\mathrm{YM}}$, введя постоянную 'т Хоофта $\curlywedge=g_{\mathrm{YM}}^{2} N$ и разложив интеграл (1.3) после перемасштабирования поля $\Phi$, мы получим в точности разложение вида (1.2), так как для графа, у которого $V$ вершин, $E$ пропагаторов и $H$ замкнутых петель или дырок, соответствующий вклад входит с весом

$$
\left(\frac{N}{\curlywedge}\right)^{V}\left(\frac{\curlywedge}{N}\right)^{E} N^{H}=N^{V-E+H} \curlywedge^{E-V} .
$$

Этот счет универсален и не зависит от конкретной модели, т.е. остается без изменений и для реалистической четырехмерной калибровочной теории с группой $S U(N)$ (прямого обобщения феноменологических групп $S U(2)$ или $S U(3))$.

Наконец, как уже было упомянуто выше, ряд по ^ или по вкладам открытых струн (при фиксированном роде $g$ ) может быть в принципе просуммирован. Это приводит к формуле

$$
F_{\text {string }}=\sum_{g=0}^{\infty} N^{2-2 g} F_{g}(\curlywedge),
$$

в которой функции $F_{g}$ могут быть проинтерпретированы как вклады рода $g$ в функцию распределения некоторой теории замкнутых струн. Более точно это означает, 
что параметр $\curlywedge$ играет роль параметра фоновой геометрии теории замкнутых струн. Очень важно отметить, что струнная интерпретация формулы (1.7) требует гладких римановых поверхностей, которые возникают только в режиме, когда существенны вклады всех диаграмм калибровочной теории, т.е. когда $\curlywedge \gg 1$ или в непертурбативной фазе калибровочной теории. Необходимо также заметить, что не существует прозрачного геометрического языка, описывающего таким же образом калибровочную теорию в режиме слабой связи, поскольку соответствующий предел теории струн чрезвычайно сингулярен ${ }^{1)}$.

Это одновременно и хорошо, и плохо. Хорошо, так как гипотеза дуальности калибровочных полей и струн позволяет в принципе, если ей доверять, предсказывать нетривиальные эффекты в непертурбативной фазе калибровочной теории, т.е. в режиме, который представляет наибольший интерес для современной теории элементарных частиц. И это плохо с той точки зрения, что становится чрезвычайно сложно проверить саму гипотезу, так как нелегко подобрать примеры, в которых обе части равенства (1.1) можно было бы вычислить независимыми способами. Именно поэтому изучение матричных интегралов (1.3) является важной задачей, так как представляет собой простейшую проверку соответствия между калибровочными полями и струнами. Как мы покажем ниже, уже в данном случае это представляет собой вполне нетривиальную проблему, с большим числом скрытых нетривиальных структур, которые не только интересны сами по себе, но и могут быть достаточно успешно применены к проверке дуальности калибровочных полей и струн в многомерном пространстве-времени, что мы и попытаемся показать в самом конце.

На $1 / N$-разложение можно также смотреть как на квазиклассическое вычисление матричного интеграла (1.3), при котором квадрат калибровочной константы связи $g_{\mathrm{YM}}^{2}=\hbar$ можно отождествить с константой связи замкнутой струны $\hbar=g_{\mathrm{s}}$ или с постоянной Планка, управляющей разложением по петлям в теории замкнутых

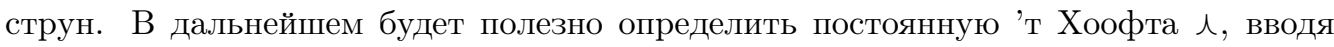
величину

$$
t_{0}=\hbar N
$$

которая, в частности, остается фиксированной и конечной в квазиклассическом пределе $N \rightarrow \infty$ одновременно с $\hbar \rightarrow 0$. При этом $1 / N$-разложение $(1.7)$ может быть эквивалентно записано как квазиклассическое:

$$
F_{\text {string }}=\sum_{g=0}^{\infty} \hbar^{2 g-2} F_{g}\left(t_{0}\right)
$$

где коэффициенты отличаются от коэффициентов в формуле (1.7) другим скейлингом по $t_{0}=\curlywedge$.

1) См., однако, работы [8], где разложение слабой связи в калибровочной теории сравнивается с непертурбативным разложением в теории струн по инстантонам на мировом листе. 
ПримеР. ГАУССов СЛУчАЙ. Для потенциала $W(\Phi)=\Phi^{2} / 2-t_{1} \Phi$ интеграл (1.3) может быть вычислен немедленно:

$$
\begin{aligned}
Z_{\text {Gauss }} & =\int d \Phi e^{-\operatorname{Tr}\left(\Phi^{2} / 2-t_{1} \Phi\right) / \hbar} \underset{\Phi \rightarrow t_{1}+\phi}{=} e^{-N t_{1}^{2} /(2 \hbar)} \int d \Phi e^{-\operatorname{Tr} \Phi^{2} /(2 \hbar)}= \\
& =C_{N} e^{-N t_{1}^{2} /(2 \hbar)}(2 \pi \hbar)^{N^{2} / 2}
\end{aligned}
$$

где $C_{N}=1 / V_{N}$. Зависимость от $N$ объема унитарной группы обеспечивает для статсуммы (1.10) то, что и производная $\frac{\partial^{2}}{\partial t_{1}^{2}} \ln Z_{\text {Gauss }}(N)$, и отношение $Z_{\text {Gauss }}(N+1) \times$ $Z_{\text {Gauss }}(N-1) / Z_{\text {Gauss }}(N)^{2}$ являются линейными функциями размера матрицы $N$. Это первое указание на появление уравнений цепочки Тоды, которые будут подробно обсуждаться ниже, в контексте матричных моделей. Квазиклассическое разложение (1.9) формулы (1.10) имеет вид

$$
F_{\text {Gauss }}=\frac{1}{\hbar^{2}}\left[\frac{t_{0}^{2}}{2}\left(\ln t_{0}-\frac{3}{2}\right)+\frac{1}{2} t_{0} t_{1}^{2}\right]+O\left(\hbar^{0}\right),
$$

где первый логарифмический член в правой части возникает из аккуратно вычисленной асимптотики объемного фактора $V_{N}$ при больших $N$. Выражение в квадратных скобках в правой части формулы (1.11) является самым тривиальным примером квазиклассических тау-функций, которые в дальнейшем будут в центре нашего рассмотрения.

\section{2. МАТРИЧНЫЕ МОДЕЛИ И ИНТЕГРИРУЕМОСТЬ}

Перед тем как обратиться к обсуждению квазиклассического предела матричного интеграла (1.3), напомним его некоторые общие свойства. $N^{2}$-кратный интеграл вычисляется с использованием калибровочной симметрии, путем сведения к $N$-кратному интегралу по собственным значениям. Ключевые же свойства последнего определяются однократным (контурным) интегралом благодаря тому, что такие интегралы непосредственно связаны с интегрируемыми системами семейства Тоды.

2.1. Матричные ансамбли и цепочка Тоды. Начнем с хорошо известного и широко используемого утверждения работы [9], заключающегося в том, что статсуммы (или производящие функции) матричных моделей удовлетворяют уравнениям иерархии Тоды, или, более конкретно, что некоторые интегралы по случайным матрицам представляют собой определенные тау-функции иерархии цепочки Тоды. Используя калибровочную инвариантность $\Phi \rightarrow U^{\dagger} \Phi U$ "односледового" потенциала (1.4) общего вида, формулу (1.3) можно переписать в виде интеграла только по собственным значениям (в настоящем разделе мы положим $\hbar=1$ ), т.е. статсумма (1.3) равна $Z=\tau_{N}(t)$, где

$$
\tau_{N}(t)=\frac{1}{N !} \int \prod_{i=1}^{N}\left(d \phi_{i} e^{-W\left(\phi_{i}\right)}\right) \Delta^{2}(\phi),
$$


a

$$
\Delta(\phi)=\prod_{i<j}\left(\phi_{i}-\phi_{j}\right)=\operatorname{det}_{i, j}\left\|\phi_{i}^{j-1}\right\|
$$

обозначает определитель Вандермонда.

Для того чтобы проверить формулу (2.1), следует применить калибровочное преобразование, которое позволяет диагонализовать матрицу $\Phi$ заменой $\Phi=U^{\dagger} \phi U$, где $U \in U(N) / U(1)^{N}$ принадлежит некоторой орбите унитарной группы $U(N)$, а $\phi=\operatorname{diag}\left(\phi_{1}, \ldots, \phi_{N}\right)$ является диагональной матрицей. Квадрат определителя Вандермонда возникает в мере интегрирования в формуле (2.1) как якобиан преобразования

$$
\left.\delta \Phi_{i j}\right|_{\Phi=\phi}=\left(U^{\dagger} \phi U-\phi\right)_{i j}=-i[\delta \Omega, \phi]_{i j}=-i \delta \Omega_{i j}\left(\phi_{i}-\phi_{j}\right),
$$

где было использовано равенство $U=e^{i \delta \Omega}$. Аналогично $(2.1)$, представления в виде интегралов по собственным значениям существуют и в многоматричных случаях, когда интегрирование производится по ансамблям случайных матриц со специальными односледовыми потенциалами. Например, в случае двухматричной модели (в "комплексно-сопряженной" версии, которая в основном и будет рассматриваться ниже) вводятся две матрицы $\Phi$ и $\Phi^{\dagger}$ с взаимодействием

$$
V\left(\Phi, \Phi^{\dagger}\right)=\Phi^{\dagger} \Phi-W(\Phi ; t)-\widetilde{W}\left(\Phi^{\dagger} ; \bar{t}\right)
$$

и матричные интегралы сводятся к интегралам по их собственным значениям

$$
\int d \Phi d \Phi^{\dagger} e^{-V\left(\Phi, \Phi^{\dagger}\right)}=\tau_{N}(t, \bar{t}),
$$

где аналогично формуле (2.1)

$$
\tau_{N}(t, \bar{t})=\frac{1}{N !} \int \prod_{i=1}^{N}\left(d^{2} z_{i} e^{-V\left(z_{i}, \bar{z}_{i}\right)}\right)|\Delta(z)|^{2}
$$

согласно формуле Хариш-Чандры-Ициксона-Зюбера. В (2.1) и (2.5) $N^{2}$-кратные матричные интегралы сведены к $N$-кратным интегралам по собственным значениям (в последнем случае интегрирование по собственным значениям $\left(z_{i}, \bar{z}_{i}\right) \in \mathbb{C} \subset \mathbb{C}^{2}$ в общем случае проводится по некоторому подмногообразию $\mathbb{C}^{2}$ вещественной размерности два), что является частным случаем часто встречающейся в калибровочных теориях процедуры локализации.

Наиболее эффективным способом вычисления интеграла по собственным значениям является метод ортогональных полиномов. Определитель Вандермонда (2.2) можно эквивалентно переписать в виде

$$
\Delta(\phi)=\operatorname{det}_{i, j}\left\|\phi_{i}^{j-1}\right\|=\operatorname{det}_{i, j}\left\|P_{j-1}\left(\phi_{i}\right)\right\|
$$

с любыми полиномами, нормированными на единичный коэффициент при старшей степени:

$$
P_{k}(\phi)=\phi^{k}+O\left(\phi^{k-1}\right),
$$


и для вычисления матричного интеграла (2.1) удобно выбрать в качестве $P_{j}(\phi)$ ортогональные полиномы с весом, определяемым потенциалом матричной модели $W(\Phi)$ :

$$
\langle i \mid j\rangle \equiv \int d \phi e^{-W(\phi)} P_{i}(\phi) P_{j}(\phi)=\delta_{i j} e^{q_{i}(t)} .
$$

Подставим определитель Вандермонда, представленный в виде (2.6), с полиномами, удовлетворяющими (2.8), в формулу (2.1). Раскрывая детерминант и используя ортогональность полиномов (2.8), производящую функцию (1.3) можно представить в виде

$$
\tau_{N}(t)=\prod_{i=0}^{N-1} e^{q_{i}(t)}=\operatorname{det}_{i, j} H_{i j}(t)
$$

т.е. в виде произведения норм полиномов (2.8) или определителя так называемой матрицы моментов [10]

$$
H_{i j}(t)=\int \phi^{i+j} e^{-W(\phi)} d \phi
$$

Из формул (2.9), (2.10) следует, что производящая функция матричной модели (1.3), (2.1), поскольку она имеет вид детерминанта оператора, экспоненциально зависящего от времен или параметров потенциала (1.4), является тау-функцией иерархии Тоды и удовлетворяет нелинейным дифференциальным уравнениям по этим временам.

Чтобы вывести эти уравнения, рассмотрим оператор Лакса цепочки Тоды, который в базисе ортогональных полиномов (2.8) имеет обычный вид трилинейной матрицы [9]:

$$
\phi P_{i}(\phi)=P_{i+1}(\phi)-p_{i}(t) P_{i}(\phi)+R_{i}(t) P_{i-1}(\phi) .
$$

Первое уравнение из серии уравнений Лакса $\partial_{t_{k}} L=\left[L, \mathcal{R} \circ L^{k}\right]$ при $k=1$

$$
\frac{\partial^{2} q_{i}}{\partial t_{1}^{2}}=e^{q_{i+1}-q_{i}}-e^{q_{i}-q_{i-1}}
$$

немедленно приводит к уравнению движения экспоненциально взаимодействующих частиц (с координатами $q_{i}$ и импульсами $p_{i}$ ), или к первому уравнению иерархии цепочки Тоды.

Действительно, пользуясь формулами (2.8), (2.11), легко получить соотношения между функциями $q_{i}(t)$ и матричными элементами $R_{i}(t)$ и $p_{i}(t)$ оператора Лакса. Сначала соотношение

$$
\langle i|\phi| i-1\rangle=e^{q_{i}(t)}=R_{i}(t) e^{q_{i-1}(t)}
$$

приводит к равенству

$$
R_{i}(t)=e^{q_{i}(t)-q_{i-1}(t)} .
$$

Дифференцируя (2.8) при $i=j$, получим

$$
\frac{\partial}{\partial t_{1}}\langle i \mid i\rangle=e^{q_{i}} \frac{\partial q_{i}}{\partial t_{1}}=\int d \phi \exp \left(-\sum t_{k} \phi^{k}\right)\left[-\phi P_{i}^{2}+2 P_{i} \frac{\partial P_{i}}{\partial t_{1}}\right]=p_{i} e^{q_{i}},
$$


где второй член в квадратных скобках пропадает благодаря условию ортогональности и условию (2.7). Далее, из формулы (2.15) следует, что

$$
p_{i}(t)=\frac{\partial q_{i}(t)}{\partial t_{1}}
$$

является просто импульсом $i$-й тодовской частицы с координатой $q_{i}(t)$. Дифференцируя теперь (2.8) при $i>j$, получаем

$$
0=\int d \phi \exp \left(-\sum t_{k} \phi^{k}\right)\left(-\phi P_{i} P_{j}+P_{j} \frac{\partial P_{i}}{\partial t_{1}}\right),
$$

а затем, используя (2.8) при сравнении этой формулы при $j \leqslant i-1$ с формулой (2.11), имеем

$$
\frac{\partial P_{i}}{\partial t_{1}}=R_{i} P_{i-1}
$$

Наконец, мы готовы продифференцировать соотношение (2.11):

$$
\phi \frac{\partial P_{i}}{\partial t_{1}}=\frac{\partial P_{i+1}}{\partial t_{1}}-\frac{\partial p_{i}}{\partial t_{1}} P_{i}+O\left(\phi^{i-1}\right) .
$$

Умножая (2.19) на $P_{i}$, интегрируя и используя соотношения $(2.8)$ и $(2.18)$, мы получим уравнение

$$
\frac{\partial p_{i}}{\partial t_{1}}=R_{i+1}-R_{i}
$$

которое с учетом (2.16) и (2.14) принимает вид уравнения движения цепочки Тоды (2.12).

Для производящей функции матричной модели (1.3), (2.1) или тау-функции цепочки Тоды (2.9) уравнение движения (2.12) может быть переписано в форме одного из бесконечного множества билинейных соотношений Хироты

$$
\frac{\partial^{2}}{\partial t_{1}^{2}} \ln \tau_{N}(t)=\frac{\tau_{N+1}(t) \tau_{N-1}(t)}{\tau_{N}(t)^{2}} .
$$

Для двухматричной модели вместо (2.8) следует ввести биортогональные полиномы

$$
\int_{\mathbb{C}} d^{2} z e^{-V(z, \bar{z})} P_{i}(z) \bar{P}_{j}(\bar{z})=\delta_{i j} e^{q_{i}(t, \bar{t})},
$$

которые аналогичным образом (см. [9]) приводят к иерархии двумеризованной решетки Тоды с временами, отвечающими потенциалу (2.4). В частности, вторую производную по времени $\partial^{2} / \partial t_{1}^{2}$ следует заменить на двумерный оператор Лапласа $\partial^{2} / \partial t_{1} \partial \bar{t}_{1}$, а первое уравнение Хироты $(2.21)$ соответственно принимает вид

$$
\frac{\partial^{2}}{\partial t_{1} \partial \bar{t}_{1}} \ln \tau_{N}(t, \bar{t})=\frac{\tau_{N+1}(t, \bar{t}) \tau_{N-1}(t, \bar{t})}{\tau_{N}(t, \bar{t})^{2}} .
$$


2.2. Матричные интегралы в переменных Мивы. Интересное явление возникает, если переписать матричный интеграл (1.3), введя переменные Мивы [11]

$$
t_{k}=t_{k}^{(0)}-\frac{1}{k} \sum_{I=1}^{L} \mu_{I}^{-k} \equiv t_{k}^{(0)}-\frac{1}{k} \operatorname{Tr} M^{-k},
$$

или параметризовать времена с помощью собственных значений вспомогательной матрицы $M$ размера $L \times L$. В качестве фоновых значений времен возьмем величины $t_{k}^{(0)}=\delta_{k, 2} / 2$, т.е. рассмотрим матричный интеграл в окрестности гауссова потенциала (1.10).

После подстановки (2.24) интеграл по собственным значениям (2.1) принимает вид [12]

$$
\begin{aligned}
Z\left(M, t^{(0)}\right) & =\frac{1}{N !} \int \prod_{i=1}^{N}\left(d \phi_{i} e^{-W\left(\phi_{i} ; t^{(0)}\right)}\right) \Delta^{2}(\phi) \prod_{i, I}\left(1-\frac{\phi_{i}}{\mu_{I}}\right)= \\
& =\frac{1}{N !} \int \prod_{i=1}^{N}\left(d \phi_{i} e^{-W\left(\phi_{i} ; t^{(0)}\right)}\right) \Delta(\phi) \frac{\Delta(\phi, \mu)}{\prod_{I} \mu_{I}^{N} \Delta(\mu)},
\end{aligned}
$$

где $\Delta(\mu)=\prod_{I<J}\left(\mu_{I}-\mu_{J}\right)$ и $\Delta(\phi, \mu)$ обозначают определители Вандермонда матриц размеров $L \times L$ и $(N+L) \times(N+L)$, соответственно. Как и в п. 2.1, удобно использовать ортогональные полиномы $(2.7),(2.8)$, которые позволяют переписать формулу (2.25) в виде

$$
\begin{aligned}
Z\left(M, t^{(0)}\right)= & \frac{1}{N ! \prod_{I} \mu_{I}^{N} \Delta(\mu)} \int \prod_{i=1}^{N}\left(d \phi_{i} e^{-W\left(\phi_{i} ; t^{(0)}\right)}\right) \times \\
& \times \operatorname{det}_{i, j}\left\|P_{j-1}\left(\phi_{i}\right)\right\| \operatorname{det}_{(i, I),(j, J)}\left\|\begin{array}{ccc}
P_{j-1}\left(\phi_{i}\right) & \ldots & P_{j-1}\left(\mu_{I}\right) \\
\vdots & \ddots & \vdots \\
P_{J-1+N}\left(\phi_{i}\right) & \ldots & P_{J-1+N}\left(\mu_{I}\right)
\end{array}\right\| .
\end{aligned}
$$

Раскрывая детерминанты матриц размеров $N \times N$ и $(N+L) \times(N+L)$ в формуле $(2.26)$ и используя, как и раньше, условие ортогональности, приходим к формуле

$$
\tau_{N}(t)=Z\left(M, t^{(0)}\right)=\frac{\tau_{N}\left(t^{(0)}\right)}{\prod_{I} \mu_{I}^{N} \Delta(\mu)} \operatorname{det}_{I, J}\left\|P_{J-1+N}\left(\mu_{I}\right)\right\|
$$

или, вводя $\psi_{J}(\mu) \equiv \mu^{-N} P_{J+N}(\mu)$,

$$
\frac{\tau_{N}(t)}{\tau_{N}\left(t^{(0)}\right)}=\frac{\operatorname{det}_{I, J}\left\|\psi_{J-1}\left(\mu_{I}\right)\right\|}{\Delta(\mu)},
$$

получаем частный случай хорошо известного представления для тау-функции иерархии Кадомцева-Петвиашвили (КП) в переменных Мивы (2.24). В гауссовом случае $t_{k}^{(0)}=\delta_{k, 2} / 2$ ортогональные полиномы могут быть отождествлены с полиномами Эрмита

$$
\psi_{J}(\mu)=\mu^{-N} \int d x e^{-x^{2}+\mu x} x^{N+J} \underset{\mu \rightarrow \infty}{\sim} \mu^{J}+\cdots,
$$


что позволяет переписать формулу (2.28) в виде матричного интеграла во внешнем матричном поле общей структуры

$$
Z(\Lambda) \propto \int d X e^{-\operatorname{Tr} \mathcal{V}(X)+\operatorname{Tr} \Lambda X},
$$

где для частного случая гауссовой модели (2.25), как следует из интегрального представления (2.29), имеют место равенства $\Lambda=M$ и $\mathcal{V}(X)=X^{2} / 2-N \ln X$. Существование двойственного представления в виде интеграла по матрицам (2.30) следует из существования другой интегрируемой структуры [13], возникающей для матричных интегралов во внешнем матричном поле.

\section{3. Интегралы Концевича, свободные фермионы и топологические}

струны. Рассмотрим теперь более детально матричную модель (2.30), где интегрирование производится по матрицам размера $L \times L$, а статсумма определена с точностью до простого (но зависящего от $M$ ) нормировочного множителя. Аналогично разложению вокруг гауссовой точки, выполненному в п. 2.2, матричный интеграл (2.30) можно рассматривать как (формальное) разложение вокруг соответствующей "классической” траектории $X=M$, определяемой уравнениями движения

$$
\Lambda=\mathcal{V}^{\prime}(M)
$$

При этом интеграл (2.30) разлагается по обратным степеням матрицы $M$, т.е. (калибровочно-инвариантная) производящая функция (2.30) рассматривается как функция переменных (2.24), а нормировка выбирается так, чтобы разложение начиналось с единицы.

И в общем случае матричный интеграл (2.30) может быть сведен к детерминантной формуле вида (2.28), которая означает, что он является тау-функцией иерархии КП (более точно, для полиномиального потенциала $\mathcal{V}(X)$ степени $p$ это $p$-я редукция Гельфанда-Дикого иерархии КП). Детерминантная формула получается [13] непосредственным вычислением интеграла

$$
\begin{aligned}
& \int d X e^{-\operatorname{Tr}(\mathcal{V}(X)-\operatorname{Tr} \Lambda X)}=\frac{1}{\Delta(\lambda)}\left(\prod_{I=1}^{L} \int d x_{I} e^{-\mathcal{V}\left(x_{I}\right)+\lambda_{I} x_{I}}\right) \Delta(x)= \\
& =\Delta^{-1}(\lambda) \Delta\left(\frac{\partial}{\partial \lambda}\right) \prod_{I} \int d x_{I} e^{-\mathcal{V}\left(x_{I}\right)+\lambda_{I} x_{I}}=\Delta^{-1}(\lambda) \operatorname{det}_{I, J}\left\|\Psi_{I-1}\left(\lambda_{J}\right)\right\|
\end{aligned}
$$

где первое равенство является следствием формулы Хариш-Чандры-ИциксонаЗюбера, а матричные элементы в правой части выражаются в терминах контурных интегралов (ср. с частным случаем (2.29))

$$
\Psi_{I}(\lambda) \equiv \int d x x^{I} e^{-\mathcal{V}(x)+\lambda x}=\left(\frac{\partial}{\partial \lambda}\right)^{I} \Psi_{0}(\lambda) .
$$

Используя при вычислениях классическое решение (2.31), вводя обозначение $\left\{\mu_{I}\right\}$ для собственных значений матрицы $M$ и восстанавливая нормировку, с учетом (2.24) 
окончательно получаем

$$
Z(M) \propto \frac{\tau_{N}(t)}{\tau_{N}\left(t^{(0)}\right)}=\frac{\operatorname{det}_{I, J} \Phi_{I-1}\left(\mu_{J}\right)}{\Delta(\mu)}, \quad I, J=1,2, \ldots, L,
$$

где

$$
\Phi_{I}(\mu)=\left(\mathcal{V}^{\prime \prime}(\mu)\right)^{1 / 2} e^{\mathcal{V}(\mu)-\mu \mathcal{V}^{\prime}(\mu)} \Psi_{I}\left(\mathcal{V}^{\prime}(\mu)\right) \underset{\mu \rightarrow \infty}{\rightarrow} \mu^{I}\left(1+O\left(\frac{1}{\mu}\right)\right),
$$

что удовлетворяет уравнениям Хироты иерархии КП вследствие простых билинейных соотношений для детерминантов (подробнее см. [13]). Детерминантная формула (2.34) верна при любых размерах матрицы $L \times L$, и в этом смысле, в отличие от матричных интегралов (1.3), (2.1), (2.5), "не чувствует" предела $L \rightarrow \infty$, который будет изучаться ниже. Теперь сразу видно, что представление (2.28) является частным случаем формулы (2.34), в котором величины (2.33) задаются выражениями (2.29).

Полученные выше формулы необходимо снабдить следующими комментариями (см. подробное их обсуждение в работах [13]).

1. Билинейные (дисперсионные!) уравнения Хироты, которым удовлетворяет детерминантная формула (2.34), эквивалентны представлению свободными полями матричных элементов

$$
\Phi_{I}(\mu) \propto\langle I|\psi(\mu) \hat{\Phi}| 0\rangle
$$

с любым оператором вида $\hat{\Phi}=\exp \left(\sum_{i, j} A_{i j} \tilde{\psi}_{i} \psi_{j}\right)$. При этом детерминантная формула (2.34) является простым следствием теоремы Вика.

2. Конкретный вид функций (2.35), обладающих простым интегральным представлением (2.33), фиксируется струнным уравнением, которое может быть записано в виде $\mathcal{L}_{-1}^{\{\mathcal{V}\}} Z=0$, с дифференциальным оператором первого порядка по временам иерархии

$$
\mathcal{L}_{-1}^{\{\mathcal{V}\}}=\sum_{n \geqslant 1} \operatorname{Tr}\left(\frac{1}{\mathcal{V}^{\prime \prime}(M) M^{n+1}}\right) \frac{\partial}{\partial t_{n}}+\frac{1}{2} \sum_{I, J}\left(\frac{1}{\mathcal{V}^{\prime \prime}\left(\mu_{I}\right) \mathcal{V}^{\prime \prime}\left(\mu_{J}\right)} \frac{\mathcal{V}^{\prime \prime}\left(\mu_{I}\right)-\mathcal{V}^{\prime \prime}\left(\mu_{J}\right)}{\mu_{I}-\mu_{J}}\right)-\frac{\partial}{\partial t_{1}},
$$

принимающим более знакомую форму одного из операторов Вирасоро $\mathcal{L}_{-1}^{\{p\}} \equiv \mathcal{L}_{-p}^{\{\mathcal{V}\}}$ для мономиальных потенциалов $\mathcal{V}(X)=X^{p+1} /(p+1)$.

3. Вообще говоря, условия Вирасоро $\mathcal{L}_{n}^{\{\mathcal{V}\}} Z=0$ при $n \geqslant-1$ могут быть использованы в качестве уравнений, определяющих (совместно с некоторыми дополнительными условиями) производящую функцию некоторой топологической теории струн [14], или $(p, q)$-минимальной струнной теории. Эквивалентно можно наложить единственное условие (2.37) вместе с полным набором билинейных уравнений Хироты ( $p$-редуцированной) иерархии КП [15]. Планарный или квазиклассический вклад в струнную статсумму при этом описывается бездисперсионной иерархией КП, которая ниже будет обсуждаться более подробно. Конкретная струнная $(p, q)$-модель при этом задается как $q$-я критическая точка $p$-редуцированной иерархии КП, которая характеризуется как решение всех приведенных выше уравнений, полученное разложением вблизи $t_{k}=p \delta_{k, p+q} /(p+q)$ в пространстве времен или констант связи. 
4. Матричные интегралы (2.30) часто интерпретируются как статсуммы топологических теорий Гинзбурга-Ландау с единственным полем и суперпотенциалом $\mathcal{V}^{\prime}(X)$, взаимодействующим с топологической гравитацией. Пространствомвременем для этих теорий является сфера с отмеченной точкой, или просто комплексная плоскость, которую удобно отождествлять с рациональной кривой $y=$ $\mathcal{V}^{\prime}(x)$, определенной аналитическим уравнением (2.31) на две комплексные переменные $(x, y)=(X, \Lambda)$. Такая интерпретация была предложена как один из примеров, определяющих непертурбативную теорию струн в терминах (квазиклассической) тау-функции, ассоциированной с комплексным многообразием, оснащенным определенной дополнительной структурой: двумя заданными функциями, или, точнее, дифференциалами $d x$ и $d y$ с фиксированными (в данном случае нулевыми) периодами. Потенциал матричной модели во внешнем поле $(2.30) \mathcal{V}=\int y d x$ при этом является интегралом от производящего дифференциала в теории Гинзбурга-Ландау. Мы увидим ниже, что данный геометрический язык является универсальным и эффективным при описании квазиклассического приближения для всех матричных интегралов.

5. Интегральное представление (2.33) можно при этом считать частным случаем преобразования дуальности [16]

$$
\Psi_{I}(y) \propto \int d \mu(x) \Psi_{I}(x) \exp \left(\int y d x\right),
$$

связывающего $(q, p)$ - и $(p, q)$-модели. Дуальные волновые функции естественным образом зависят от дуальных координат $x$ и $y$, соответственно. На классическом уровне эта дуальность, таким образом, имеет вид замены локальной координаты, и мы увидим ниже, что это есть проявление общего свойства квазиклассической формулировки матричных моделей.

\section{3. ПЛАНАРНЫЙ ПРЕДЕЛ ОДНОМАТРИЧНОЙ МОДЕЛИ}

Вернемся теперь к простейшему матричному интегралу (1.3) и перейдем к непосредственному обсуждению планарного предела $N \rightarrow \infty, \hbar \rightarrow 0$ при фиксированном введенном в (1.8) параметре $t_{0}$. В пределе $N \rightarrow \infty$ из всего топологического разложения (1.7) остается лишь первый член $F_{0}=\mathcal{F}$ с $g=0$, отвечающий суммированию по планарным ленточным графам, которые могут быть изображены на сфере без самопересечений. Одновременно в этом пределе, поскольку $\hbar \rightarrow 0$, матричный интеграл (1.3) может быть вычислен квазиклассически, т.е. методом стационарной фазы. Изучение экстремумов непосредственно в интеграле по матрицам (1.3) бессмысленно из-за огромного калибровочного вырождения экстремальных конфигураций в пространстве всех матриц, поэтому ниже мы сразу приступаем к изучению квазиклассического поведения интегралов по собственным значениям (2.1) и (2.5).

3.1. Интеграл по собственным значениям и комплексная кривая. Квазиклассическое значение интеграла по собственным значениям (2.1) при $\hbar \rightarrow 0$ может быть получено путем изучения экстремумов эффективного потенциала

$$
W_{\text {eff }}(\phi)=W(\phi)-2 \hbar \ln \Delta(\phi),
$$


или, точнее, решением уравнения стационарности

$$
W^{\prime}\left(\phi_{j}\right)=2 \hbar \sum_{k \neq j} \frac{1}{\phi_{j}-\phi_{k}} .
$$

При $\hbar=0$ взаимодействие в правой части уравнения (3.2) "выключено", и все собственные значения $\phi_{j}$ распределены некоторым образом по минимумам, или, в наиболее общей постановке задачи, по всем экстремумам, в которых $W^{\prime}(\phi)=0$. Более удобно при $N \rightarrow \infty$ ввести плотность собственных значений

$$
\rho(x)=\hbar \sum_{j=1}^{N} \delta\left(x-\phi_{j}\right)
$$

или резольвенту

$$
G(x)=\hbar\left\langle\operatorname{Tr} \frac{1}{x-\Phi}\right\rangle_{\Phi}=\int_{\mathbf{C}} \frac{\rho(\phi) d \phi}{x-\phi},
$$

определенную на $x$-плоскости с вырезанным носителем собственных значений, или, как мы увидим ниже, на двойном накрытии комплексной $x$-плоскости, разрезанной вдоль некоторых отрезков $\mathbf{C}=\bigcup_{j} C_{j}$, изображенных на рис. 1 , и нормированную

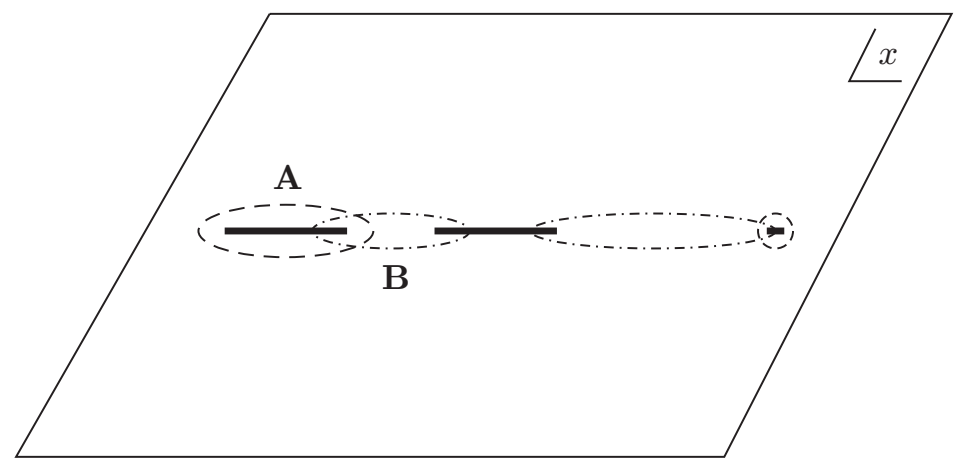

Рис. 1. Разрезы в плоскости собственных значений и циклы на $x$-листах гиперэллиптической римановой поверхности (3.10) одноматричной модели. А-циклы традиционно выбираются вокруг разрезов или носителей собственных значений (которые в данном случае предполагаются отрезками на вещественной оси $\operatorname{Im} x=0)$, в то время как В-циклы связывают два различных носителя собственных значений.

следующим образом:

$$
\frac{1}{2 \pi i} \oint_{\mathbf{C}} G(x) d x=\int_{\mathbf{C}} \rho(\phi) d \phi=t_{0} .
$$

В этих терминах соотношение (3.2) может быть записано как интегральное уравнение:

$$
W^{\prime}(x)=2 f_{\mathbf{C}} \frac{\rho(\phi) d \phi}{x-\phi}=G\left(x_{+}\right)+G\left(x_{-}\right), \quad x \in \forall C_{j} \subset \mathbf{C},
$$


где $x_{ \pm}=x \pm i 0$ представляют собой две близкие точки на двух различных сторонах разреза - выше и ниже разреза, если распределение собственных значений вещественно и разрезы вытянуты вдоль вещественной оси. Это уравнение выполняется в любой точке носителя собственных значений $\mathbf{C}$, состоящего из нескольких несвязных областей для общего полиномиального потенциала. Формула (3.6) может быть далее переписана как алгебраическое уравнение на резольвенту $G$ (см., например, [17]--[20]):

$$
G^{2}-W^{\prime}(x) G=f(x)
$$

где $f(x)$ в правой части обозначает полином степени на единицу меньше, чем степень $W^{\prime}(x)$. В самом деле, из уравнения (3.6) следует, что

$\left.\left(G^{2}-W^{\prime} G\right)\right|_{x=x_{+}}-\left.\left(G^{2}-W^{\prime} G\right)\right|_{x=x_{-}}=\left(G\left(x_{+}\right)-G\left(x_{-}\right)\right)\left(G\left(x_{+}\right)+G\left(x_{-}\right)-W^{\prime}(x)\right)=0$,

т.е. левая часть формулы (3.7) представляет собой однозначную функцию $f(x)$, свойства которой (то, что она является полиномом определенной степени) фиксируются поведением при $x \rightarrow \infty$.

Уравнение (3.7) определяет гиперэллиптическую кривую, и, как мы увидим ниже, квазиклассическая свободная энергия $\mathcal{F}=F_{0}$ (первый член в правой части формулы (1.2)) может быть целиком определена в терминах кривой (3.7) и производящего дифференциала $G d x$, определяемого резольвентой (3.4) и определяющего распределение собственных значений. Для полиномиальных потенциалов уравнение (3.7) описывает алгебраическую кривую (конечного рода $n$ для потенциала $W$ степени $n+1)$ и определяет резольвенту $G$ как алгебраическую функцию на кривой, дважды накрывающей $x$-плоскость ${ }^{2)}$.

Вводя новую переменную с помощью

$$
y=W^{\prime}(x)-2 G,
$$

уравнение (3.7) можно переписать в виде

$$
y^{2}=W^{\prime}(x)^{2}+4 f(x)=R(x)
$$

где $R(x)$ - полином степени $2 n$ для потенциала $W(x)$ степени $n+1$. С таким полиномом уравнение (3.10) является каноническим представлением гиперэллиптической кривой рода $g=n-1$.

3.2. Свободная энергия: геометрическое определение. Под вычислением свободной энергии матричной модели (1.3) в квазиклассическом пределе или в пределе больших $N$ обычно понимают решение вариационной задачи (3.2) для

2) Если, однако, допустить всевозможные длинные операторы $\operatorname{Tr} \Phi^{L}$ с $L \rightarrow \infty$, то эта кривая превращается в кривую бесконечного рода, и ничего нельзя сразу утверждать о свойствах резольвенты $G$. 
функционала ${ }^{3)}$

$$
\begin{aligned}
\mathcal{F}=- & {\left[\sum_{i} W\left(\phi_{i}\right)-\hbar \sum_{i \neq j} \ln \left|\phi_{i}-\phi_{j}\right|\right]_{N=\sum_{\alpha} N_{\alpha}}=} \\
=- & {\left[\int d x \rho(x) W(x)-\int d x_{1} \int d x_{2} \rho\left(x_{1}\right) \ln \left|x_{1}-x_{2}\right| \rho\left(x_{2}\right)+\right.} \\
& \left.+\sum_{\alpha} \Pi_{\alpha}\left(\int_{C_{\alpha}} d x \rho(x)\right)-S_{\alpha}\right]_{\frac{\delta \mathcal{F}}{\delta \rho}=0} .
\end{aligned}
$$

В дополнение к формальному определению (2.1) здесь следует добавить некоторое фиксированное разбиение всех $N \rightarrow \infty$ собственных значений по некоторому (конечному) числу групп с числами заполнения $\sum_{\alpha} N_{\alpha}=N$, что приводит к дополнительному линейному соотношению на новые квазиклассические переменные $\sum_{\alpha} S_{\alpha}=\hbar N=t_{0}$. Обсудим сначала природу и смысл этих групп и соответствующих им чисел заполнения.

Интуитивно ясно, что в квазиклассическом пределе собственные значения выпадают в конденсаты, которые могут быть расположены в окрестности любого из минимумов (или даже около любого экстремума $W^{\prime}(\phi)=0$, как мы увидим ниже). Если бы кулоновское отталкивание, происходящее из детерминанта Вандермонда, было "выключено" при $\hbar \rightarrow 0$, собственные значения расположились бы непосредственно в экстремумах. Поэтому для малых чисел заполнения собственных значений эта картина очевидна, при больших значениях она несколько усложняется (конденсаты могут расти и сталкиваться), и мы обсудим некоторые детали этого процесса ниже. Скачки же между конденсатами подавлены экспоненциально, поскольку их вклад порядка $e^{- \text {const/ } /} \sim e^{- \text {const. } N}$, а следовательно, естественно ввести дополнительные (по отношению к коэффициентам потенциала (1.4)) переменные, или фиксировать числа заполнения

$$
S_{\alpha}=\hbar N_{\alpha}=\int_{C_{\alpha}} \rho(x) d x=\frac{1}{2 \pi i} \oint_{C_{\alpha}} G d x
$$

в каждом экстремуме при решении вариационной задачи для функционала (3.11), например, вводя соответствующие множители Лагранжа $\Pi_{\alpha}$.

Более того, понятно, что в отсутствие кулоновского взаимодействия стационарные конфигурации в общем положении представляют собой наборы изолированных точек - экстремумов потенциала $W^{\prime}(\phi)=0$, входящих при больших $N$ с определенными кратностями. Однако взаимодействие сглаживает картину, и вместо изолированных точек возникают одномерные линии, на которые можно смотреть как на аналоги траекториям в механике или как на аналоги изинговых струн. При аналитическом продолжении производящей функции в пространстве параметров и деформации контуров интегрирования это приводит к появлению комплексных кривых статистических матричных ансамблей.

3) Под модулем в аргументе логарифма обычно понимается выбор подходящей ветви логарифмической функции, это буквально совпадает с модулем в случае, когда все собственные значения предполагаются вещественными. 


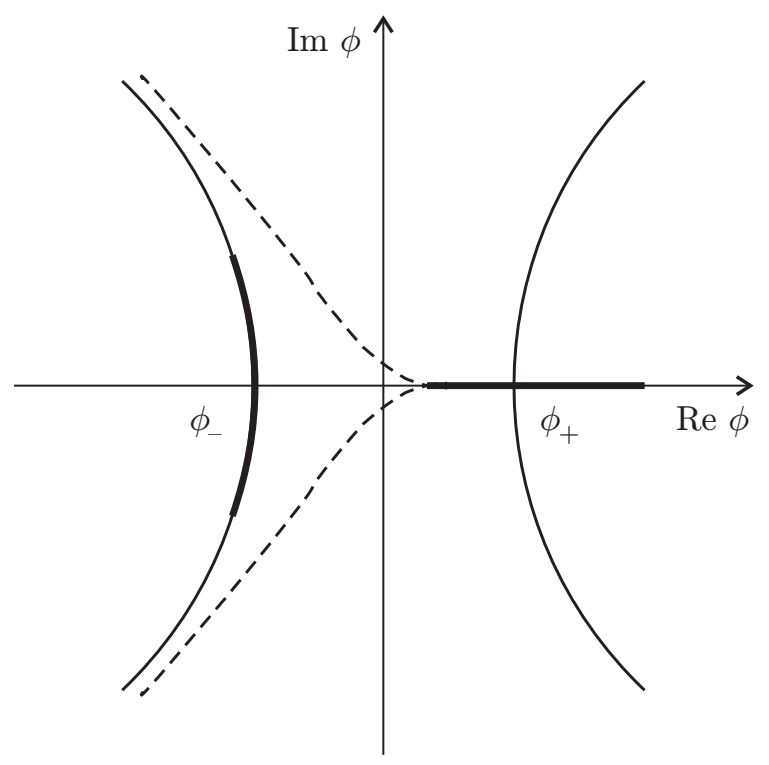

Рис. 2. Комплексная геометрия потенциала $W(\phi)=\phi^{3} / 3-t \phi$. Линии уровня, проходящие через критические точки $\phi_{ \pm}= \pm \sqrt{t}$, описываются уравнениями $\operatorname{Im} \phi=0$ и $(\operatorname{Re} \phi)^{2}-(\operatorname{Im} \phi)^{2} / 3-t=0$. Кулоновское отталкивание растягивает собственные значения вдоль сегментов линий уровня вокруг критических точек.

Чтобы понять это лучше, следует вспомнить, что интеграл по собственным значениям (2.1), как и любой другой, зависит от выбора контуров интегрирования. Для потенциала степени $n+1$ существует $n$-мерный базис в линейном пространстве всевозможных контуров, для которых интеграл по каждому собственному значению сходится. Легко видеть, что каждый контур скорейшего спуска может быть выбран в комплексной плоскости так, что он будет проходить только через одну из $n$ экстремальных точек $W^{\prime}(\phi)=0$ (подробное обсуждение этого вопроса можно найти в [21]).

Для простейшего негауссова потенциала $W(\phi)=\phi^{3} / 3-t \phi$ соответствующая геометрия проиллюстрирована на рис. 2. Критическими точками потенциала являются точки $\phi_{ \pm}= \pm \sqrt{t}$ (на рис. 2 параметр $t$ выбран вещественным и положительным). Линии скорейшего спуска для $\operatorname{Re} W$, проходящие через критические точки $\phi_{ \pm}$, являются линиями уровня для $\operatorname{Im} W=\operatorname{Im} \phi\left((\operatorname{Re} \phi)^{2}-(\operatorname{Im} \phi)^{2} / 3-t\right)=0$. Контуры интегрирования, для которых интегралы по собственным значениям сходятся, должны начинаться и заканчиваться в одном из $n+1$ секторов комплексной $\phi$-плоскости, где $\operatorname{Re} W>0$ (на рис. $2 n=2$, т.е. в этом случае существуют три таких сектора). Поскольку подынтегральное выражение голоморфно и значение интеграла не зависит от локальной деформации контуров, метод скорейшего спуска "выбирает" контур интегрирования из одного допустимого сектора в другой, проходящий через критическую точку (два таких возможных контура показаны на рис. 2 штриховыми 
линиями). Как уже отмечалось выше, вклад определителя Вандермонда в эффективный потенциал (3.1) приводит к отталкиванию собственных значений, так что из положений точно в экстремумах $W^{\prime}(x)=0$ они "растекаются" вдоль сегментов допустимых контуров (см. рис. 2).

Квазиклассическая свободная энергия (3.11) вычисляется при критических плотностях, т.е. когда

$$
\frac{\delta \mathcal{F}}{\delta \rho(x)}=-W(x)+2 \int_{\mathbf{C}} d \phi \rho(\phi) \ln |x-\phi|-\Pi_{\alpha}=0, \quad x \in C_{\alpha} .
$$

Правое равенство может быть использовано для вычисления множителей Лагранжа $\Pi_{\alpha}$, которые будут интенсивно использоваться ниже, так как они равны частным производным свободной энергии (3.11) по числам заполнения (3.12):

$$
\frac{\partial \mathcal{F}}{\partial S_{\alpha}}=\Pi_{\alpha}+\int_{\mathbf{C}} d x \frac{\delta \mathcal{F}}{\delta \rho(x)} \frac{\partial \rho(x)}{\partial S_{\alpha}}=\Pi_{\alpha},
$$

поскольку вариационная производная обращается в нуль согласно формуле (3.13), которая одновременно определяет и сам множитель Лагранжа ${ }^{4)}$.

Таким образом, формула (3.11) может рассматриваться как независимое геометрическое определение планарной свободной энергии $\mathcal{F}$. Чтобы сделать его точным, решение уравнений (3.13), (3.11) следует дополнить граничными условиями - выбором носителя $\mathbf{C}$, на котором плотность собственных значений $\rho(x) \neq 0$, или области неаналитичности резольвенты $G(x) d x(3.4)$. Этот носитель (набор разрезов для одноматричной модели или “капель" в двумерном случае модели (2.5) (см. рис. 1, 2)) вводится "руками", но их длина и форма (или размер и форма в двумерном случае, который будет подробно обсуждаться ниже) после этого определяются динамически. Плотность собственных значений в одномерной области (объединение разрезов) определяется с помощью уравнения (3.7) через параметры потенциала и коэффициенты вспомогательной функции

$$
f(x)=\sum_{k=0}^{n-1} f_{k} x^{k}
$$

связанные с числами заполнения собственных значений. Отметим сразу, что свобода в выборе дополнительных свободных параметров полинома (3.15), появляющегося в правой части уравнения (3.7), в точности поглощается введенными новыми параметрами - числами заполнения (3.12).

Ниже мы продемонстрируем, что геометрическое определение свободной энергии (3.14) в точности совпадает с частным случаем так называемых препотенциалов или квазиклассических тау-функций, которые в принципе могут быть введены для любого общего комплексного многообразия, оснащенного некоторой дополнительной структурой, информация о которой обычно содержится в мероморфной дифференциальной форме. Для планарного предела одноматричной модели, который

\footnotetext{
4)Заметим также, что множители Лагранжа в формуле (3.11) необходимы для выполнения бездисперсионных уравнений Хироты и их аналогов, т.е. они играют ту же роль, что и аккуратная нормировка меры (1.5), фиксируемая соотношениями (2.21), (2.23).
} 
мы обсуждали выше, это комплексное многообразие может быть отождествлено с гиперэллиптической кривой (3.7), (3.10).

ПримеР. ГАУССОв СЛУЧАЙ. Решим теперь явно уравнение (3.6) для простейшего гауссова потенциала $W=x^{2} / 2$. Правая часть уравнения (3.7), или полином (3.15), в данном случае $(n=1)$ является просто константой, и решение уравнения $(3.7)$ имеет вид

$$
G=\frac{x}{2}+\frac{1}{2} \sqrt{x^{2}+4 f} \equiv \frac{x}{2}+\frac{1}{2} \sqrt{x^{2}-a^{2}},
$$

т.е. у него один разрез в плоскости собственных значений, с единственным параметром $a^{2}=-4 f$, который можно отождествить с квадратом половины длины разреза, расположенного симметрично вокруг точки $x=0$ в $x$-плоскости. Его можно зафиксировать с помощью нормировки (3.5), что приводит к значению $a^{2}=4 t_{0}$. В этом случае кривая матричной модели (3.7), (3.10) рациональна или ее род $g=0, \mathrm{a}$ плотность собственных значений равна

$$
\rho(x)=\frac{1}{2 \pi} \sqrt{a^{2}-x^{2}} .
$$

Легко убедиться, что статсумма в данном случае выражается формулой

$$
\left.\mathcal{F}\right|_{t_{1}=0}=\left.\left(\frac{t_{0}^{2}}{2}\left(\ln t_{0}-\frac{3}{2}\right)+\frac{1}{2} t_{0} t_{1}^{2}\right)\right|_{t_{1}=0}=\frac{t_{0}^{2}}{2}\left(\ln t_{0}-\frac{3}{2}\right)
$$

совпадающей с первым членом квазиклассического разложения выражения (1.10) при $t_{1}=0$, отвечающим случаю симметрично расположенного разреза $\mathbf{C}=[-a, a]$ в $x$-плоскости и вигнеровской плотности собственных значений (3.17). Формула (3.18) следует из единственного в гауссовом случае соотношения (3.14) для производной свободной энергии по полному числу собственных значений $S=t_{0}$

$$
\frac{\partial \mathcal{F}}{\partial t_{0}}=-W\left(x^{*}\right)+2 \int_{\mathbf{C}} d \phi \rho(\phi) \ln \left|x^{*}-\phi\right|=\frac{2}{\pi} \int_{0}^{a} \sqrt{a^{2}-x^{2}} \ln x d x=t_{0}\left(\ln t_{0}-1\right),
$$

где были использованы равенства $x^{*}=0$ и $4 a^{2}=t_{0}$. Интегрирование (3.19) приводит к выражению (3.18), в котором зависимость от $t_{1}$ тривиально восстанавливается благодаря простому аргументу со сдвигом (например, из формулы (1.10) немедленно следует, что $Z_{\text {Gauss }}=\left.e^{-t_{0} t_{1}^{2} /\left(2 \hbar^{2}\right)} Z_{\text {Gauss }}\right|_{t_{1}=0}$.

\section{4. КВАЗИКЛАССИЧЕСКАЯ ТАУ-ФУНКЦИЯ}

Квазиклассическая свободная энергия матричной модели, определенная формулой (3.14), является частным случаем так называемых препотенциалов, или, более точно, квазиклассических тау-функций, введенных в работе [22]. Чтобы продолжить обсуждение геометрических свойств матричных моделей, нам следует узнать больше о свойствах квазиклассических тау-функций, вспомнив сначала некоторые необходимые факты из геометрии комплексных кривых. 


\section{1. Римановы поверхности, голоморфные и мероморфные дифферен-}

циалы. Начнем с того, что вспомним некоторые хорошо известные факты из теории римановых поверхностей или одномерных комплексных многообразий - комплексных кривых. Топология компактной ориентируемой римановой поверхности характеризуется единственным целым неотрицательным числом - родом $g$, обычно представляемым как число "ручек", приклеенных к сфере. Простейшими примерами являются собственно сфера без "ручек" $(g=0)$ и тор $(g=1)$. Пример римановой поверхности с $g=3$ изображен на рис. 3 .

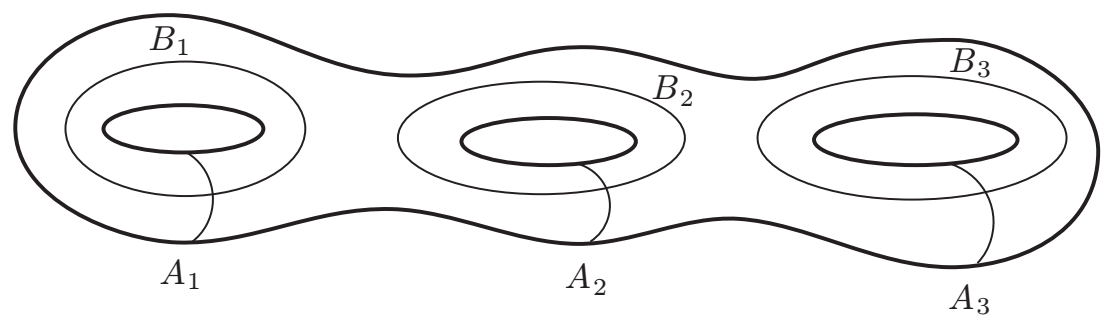

Рис. 3. Компактная риманова поверхность рода $g=3$ с выбранным базисом $\mathbf{A}$ - и В-циклов, с формой пересечений $A_{\alpha} \circ B_{\beta}=\delta_{\alpha \beta}$. Переобозначение

A- и В-циклов называется преобразованием дуалъности.

На римановой поверхности $\Sigma_{g}$ рода $g$ существуют $2 g$ независимых нестягиваемых контуров, которые можно разбить на пары, обычно называемые А- и Вциклами, $\mathbf{A} \equiv\left\{A_{\alpha}\right\}, \mathbf{B} \equiv\left\{B_{\alpha}\right\}$ (см. рис. 3 ), $\alpha=1,2, \ldots, g$, с формой пересечений $A_{\alpha} \circ B_{\beta}=\delta_{\alpha \beta}$. Базис в пространстве 1-форм естественным образом двойствен базису одномерных циклов, например голоморфные (или абелевы первого рода) дифференциалы $\bar{\partial}\left(d \omega_{\alpha}\right)=0$ канонически выбираются нормированными на А-циклы:

$$
\oint_{A_{\beta}} d \omega_{\alpha}=\delta_{\alpha \beta}
$$

Их интегралы вдоль В-циклов образуют при этом матрицу периодов

$$
T_{\alpha \beta}=\oint_{B_{\beta}} d \omega_{\alpha}
$$

(симметричную матрицу размера $g \times g$ с положительно определенной мнимой частью), которая является одной из наиболее важных характеристик комплексной кривой. Симметричность матрицы периодов следует из билинейных соотношений Римана для голоморфных дифференциалов

$$
0=\int_{\Sigma} d \omega_{\beta} \wedge d \omega_{\gamma}=\sum_{\alpha}\left(\oint_{A_{\alpha}} d \omega_{\beta} \oint_{B_{\alpha}} d \omega_{\gamma}-\oint_{A_{\alpha}} d \omega_{\gamma} \oint_{B_{\alpha}} d \omega_{\beta}\right)=T_{\beta \gamma}-T_{\gamma \beta}
$$

являющихся следствием теоремы Стокса на разрезанной римановой поверхности (см. рис. 4), и из обращения в нуль любой $(2,0)$-формы, в частности $d \omega_{\beta} \wedge d \omega_{\gamma}$, 


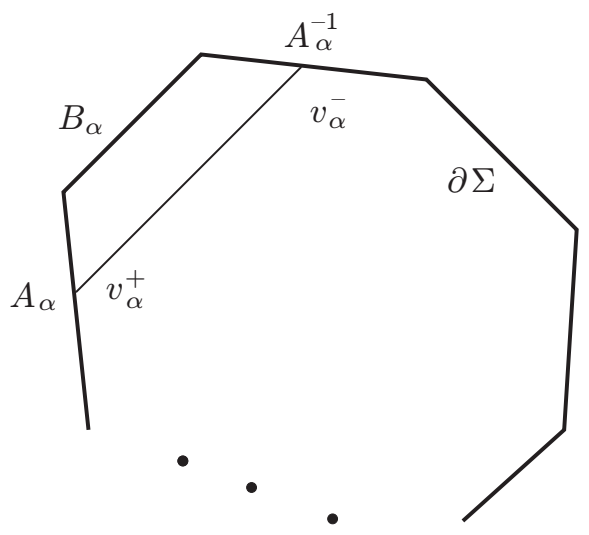

Рис. 4. Разрезанная риманова поверхность с границей $\partial \Sigma$. Интеграл по границе может быть разделен на несколько вкладов (см. ниже формулу (4.34)). В процессе вычисления мы использовали то, что граничные значения абелевых интегралов $v_{\alpha}^{ \pm}$на двух границах разреза различаются на период соответствующего дифференциала $d \omega_{\alpha}$ по двойственному циклу.

на одномерном комплексном многообразии. Аналогичным образом мнимая часть матрицы периодов положительно определена, поскольку

$$
\operatorname{Im} T_{\alpha \beta} \propto \int_{\Sigma} d \omega_{\alpha} \wedge d \bar{\omega}_{\beta} .
$$

В отличие от голоморфных дифференциалов, мероморфные дифференциалы $d \Omega$ аналитичны везде, кроме конечного числа точек $P$, где они могут иметь полюсы и $\bar{\partial} d \Omega \propto \delta(P)$. Их канонический вид фиксируется не только половиной периодов (как в случае голоморфных дифференциалов, см. (4.1)), но также их поведением (главной частью) в сингулярных точках. Стандартная классификация мероморфных дифференциалов включает абелевы дифференциалы второго рода с единственной сингулярностью в некоторой точке $P_{0}$ вида $d \Omega_{k} \sim d \zeta / \zeta^{k+1}+\cdots, k \geqslant 1$, где многоточием обозначена несингулярная часть, а $\zeta$ является локальной координатой в точке $P_{0}: \zeta\left(P_{0}\right)=0$. Заметим, что этот ряд начинается с дифференциалов с полюсами второго порядка, так как не существует дифференциала с единственным полюсом первого порядка, из-за равенства нулю полного вычета. Вместо этого, для того чтобы учесть полюсы первого порядка, удобно ввести абелевы дифференциалы третьего рода $d \Omega_{ \pm}$с двумя полюсами первого порядка в некоторых точках $P_{ \pm}$ и противоположными по знаку единичными вычетами в этих точках.

Заметим теперь, что к определенному таким образом абелеву дифференциалу второго и/или третьего рода всегда можно добавить любую линейную комбинацию голоморфных дифференциалов, не меняя поведения в особых точках. Чтобы зафиксировать эту неопределенность, следует наложить $g$ условий (размерность пространства голоморфных дифференциалов) на их периоды, каноническим выбором таких условий является равенство нулю всех $\mathbf{A}$-периодов, $\oint_{\mathbf{A}} d \Omega=0$. Это означает, 
что в качестве строгого определения абелевых дифференциалов следует написать

$$
d \Omega_{k} \underset{P \rightarrow P_{0}}{\sim} \frac{d \zeta}{\zeta^{k+1}}+\cdots, \quad \oint_{\mathbf{A}} d \Omega_{k}=0, \quad k \geqslant 1,
$$

для второго рода и

$$
d \Omega_{0} \underset{P \rightarrow P_{ \pm}}{\sim} \pm \frac{d \zeta_{ \pm}}{\zeta_{ \pm}}+\cdots, \quad \oint_{\mathbf{A}} d \Omega_{0}=0,
$$

для дифференциалов третьего рода, где $\zeta_{ \pm}\left(P_{ \pm}\right)=0$. Бипольный дифференциал (4.5) может быть также представлен в виде

$$
d \Omega_{0}=d \ln \frac{E\left(P, P_{+}\right)}{E\left(P, P_{-}\right)},
$$

где $E\left(P, P^{\prime}\right)$ - так называемая главная форма на $\Sigma \times \Sigma$, или $(-1 / 2)$-бидифференциал с единственным нулем при совпадающих аргументах (подробнее его свойства см. в книге [23]).

Таким же способом, как для (4.3), можно выписать билинейные соотношения Римана для общих мероморфных абелевых дифференциалов. Например, для абелевых дифференциалов первого и третьего рода получаем соотношение

$$
\begin{aligned}
0=\int_{\Sigma} d \omega_{\beta} \wedge d \Omega_{0}=\sum_{\alpha}\left(\oint_{A_{\alpha}} d \omega_{\beta} \oint_{B_{\alpha}} d \Omega_{0}-\oint_{A_{\alpha}} d \Omega_{0} \oint_{B_{\alpha}} d \omega_{\beta}\right)+ \\
\quad+\left.\operatorname{res}\right|_{\infty}\left(d \omega_{\beta}\right) \int_{\infty_{-}}^{\infty_{+}} d \Omega_{0}-\left.\operatorname{res}\right|_{\infty}\left(d \Omega_{0}\right) \int_{\infty_{-}}^{\infty_{+}} d \omega_{\beta}=\oint_{B_{\beta}} d \Omega_{0}-\int_{\infty_{-}}^{\infty_{+}} d \omega_{\beta},
\end{aligned}
$$

которое широко используется в теории интегрируемых систем Тоды, естественно и часто возникающих в контексте матричных моделей.

На практике комплексные многообразия могут быть эффективно описаны системами полиномиальных уравнений в многомерных комплексных евклидовых пространствах $\mathbb{C}^{K}$. Чтобы получить комплексную кривую, следует наложить $K-1$ уравнений на $K$ переменных. Коэффициенты этих уравнений играют роль модулей комплексной структуры соответствующей римановой поверхности. Для поверхности рода $g>1$ комплексная размерность пространства модулей равна $\operatorname{dim}_{\mathbb{C}} \mathcal{M}_{g}=$ $3 g-3$, что может быть найдено из теоремы Римана-Роха. Римановы поверхности с различными комплексными структурами не могут быть голоморфно отображены друг на друга, т.е. являются различными комплексными многообразиями, хотя и могут быть изоморфны как вещественные поверхности. Заметим также, что это говорит о том, что матричные элементы матрицы периодов (4.2) не являются функционально независимыми, так как они представляют собой $g(g+1) / 2$ функций от всего лишь $3 g-3$ модулей, за исключением родов $g=2,3$, которые являются решением квадратного уравнения $g(g+1) / 2=3 g-3$, и исключительного случая $g=1$ с единственным модулем комплексного тора.

4.2. Определение квазиклассической тау-функции. Пусть $\Sigma$ - риманова поверхность некоторого конечного рода $g$. Предположим, что на ней заданы два мероморфных дифференциала, обозначенные $d x$ и $d y$, с фиксированными периодами. 
Как уже обсуждалось выше, без потери общности в качестве таких дифференциалов всегда можно взять канонически нормированные мероморфные дифференциалы, например с нулевыми А-периодами. Однако, если одновременно зафиксировать оставшиеся В-периоды, это приведет к нетривиальным ограничениям на пространстве модулей $\Sigma$. На практике это ограничит нас на некоторое подпространство полного пространства модулей, а размерность этого подпространства можно оценить следующим образом. Дифференциалы $d x$ и $d y$ определены с точностью до умножения на число, и фиксация $\mathbf{B}$-периодов $d x$ дает $g-1$ условий (с учетом свободы относительно растяжений), в то время как $\mathbf{B}$-периоды $d y$ приводят к $g-2$ дополнительным условиям. Таким образом, мы получаем систему с $(3 g-3)-(g-1)-(g-2)=g$ параметрами, т.е. с числом модулей, равным роду кривой $\Sigma$.

Комплексная кривая, оснащенная двумя мероморфными дифференциалами с фиксированными периодами, или, более кратко, $g$-параметрическое семейство кривых, определяет интегрируемую систему5). На практике обычно рассматривается $g$-параметрическое семейство римановых поверхностей $\Sigma$, оснащенных производящим мероморфным дифференциалом, определяемым с точностью до константы формулой

$$
d S \propto y d x, \quad \delta_{\text {moduli }} d S=\text { holomorphic },
$$

где $y(P)=\int^{P} d y, \quad P \in \Sigma$, представляет собой абелев интеграл на $\Sigma$, а под вариацией по модулям понимается любая локальная вариация оставшихся $g$ параметров семейства, которое остается после фиксации всех периодов $d x$ и $d y$.

Общее определение препотенциала интегрируемой системы для $g$-мерного семейства гладких кривых $\Sigma_{g}$ рода $g$, оснащенных мероморфными 1-формами, имеет вид

$$
\begin{aligned}
\mathbf{S} & =\oint_{\mathbf{A}} d S, \\
\frac{\partial \mathcal{F}}{\partial \mathbf{S}} & =2 \pi i \oint_{\mathbf{B}} d S,
\end{aligned}
$$

где $\mathbf{A}$ и $\mathbf{B}$ - дуальные циклы в базисе гомологий. Препотенциал определяется формулой (4.9) локально на пространстве модулей $\Sigma$, или, точнее, на пространстве Тейхмюллера при фиксированном базисе в $H^{1}(\Sigma, \mathbb{Z})$. Для комплексных кривых, когда к формулам (4.9) естественно добавить переменные, связанные с вырожденными циклами или отмеченными точками (как в простейшем примере гауссовой модели (3.18)), точное определение обобщенного таким образом препотенциала было предложено в работе [22], где он назывался логарифмом квазиклассической тау-функции или тау-функции обобщенной иерархии Уизема. Непротиворечивость формул (4.9) гарантируется условием интегрируемости

$$
\frac{\partial^{2} \mathcal{F}}{\partial S_{\alpha} \partial S_{\beta}}=2 \pi i T_{\alpha \beta},
$$

5) В наиболее общем духе теоремы Лиувилля можно сказать, что на $g$-мерном подпространстве пространства модулей $\Sigma$ можно всегда выбрать $g$ независимых функций - гамильтонианов или действий, в то время как координаты на якобиане Јас кривой $\Sigma$, т.е. на $g$-мерном комплексном торе, играют роль комплексифицированных угловых переменных. 
следующим непосредственно из (4.8) и (4.3), или условием симметричности матрицы периодов $T_{\alpha \beta}$ римановой поверхности $\Sigma_{g}$.

Для вырожденных кривых или кривых с отмеченными точками формулы (4.9) следует рассматривать более аккуратно и не всегда применять непосредственно. Вырожденный А-цикл превращается в пару отмеченных точек $P_{ \pm}$(солитонное вырождение "ручки"), и для такой вырожденной "ручки" формулы (4.9) преобразуются B

$$
\begin{gathered}
t_{0}=\left.\frac{1}{2 \pi i} \operatorname{res}\right|_{P_{+}} d S=-\left.\frac{1}{2 \pi i} \operatorname{res}\right|_{P_{-}} d S, \\
\frac{\partial \mathcal{F}}{\partial t_{0}}=4 \pi i \int_{P_{-}}^{P_{+}} d S
\end{gathered}
$$

где последнее выражение, вообще говоря, расходится и должно применяться только после аккуратного доопределения, при котором расходящийся интеграл заменяется на конечную величину, как это имело место в простейшем гауссовом примере (3.19).

В наиболее общей постановке определение (4.9) должно быть дополнено временны́ми переменными, ассоциированными с абелевыми дифференциалами второго рода, с сингулярностями в некоторой точке:

$$
\begin{array}{ll}
t_{k}=\left.\frac{1}{2 \pi i k} \operatorname{res}\right|_{P_{0}} \xi^{-k} d S, & k>0, \\
\frac{\partial \mathcal{F}}{\partial t_{k}}=\left.\frac{1}{2 \pi i} \operatorname{res}\right|_{P_{0}} \xi^{k} d S, & k>0,
\end{array}
$$

где $\xi$ обозначает обратную локальную координату в окрестности $P_{0}: \xi\left(P_{0}\right)=\infty$. Условие согласованности для (4.12) обеспечивается соотношением

$$
\frac{\partial^{2} \mathcal{F}}{\partial t_{n} \partial t_{k}}=\left.\frac{1}{2 \pi i} \operatorname{res}\right|_{P_{0}}\left(\xi^{k} d \Omega_{n}\right),
$$

а симметричность (4.13) следует из равенства $\Omega_{n}=\xi_{+}^{n}$, где индекс + означает главную, сингулярную в точке $P_{0}$, часть функции $\xi^{n}$. Другими словами, дифференциал $d \Omega$ мероморфен на поверхности $\Sigma_{g}$ с единственной сингулярностью в точке $P_{0}$, т.е. является абелевым дифференциалом второго рода (4.4).

Таким образом, базис времен $\left(\mathbf{S}, \mathbf{t}, t_{0}\right)$ в точности отвечает базису абелевых дифференциалов первого, второго и третьего рода. Для производящей 1-формы (4.8) имеем

$$
\begin{aligned}
& \frac{\partial d S}{\partial S_{\alpha}}=d \omega_{\alpha}, \quad \alpha=1,2, \ldots, g, \\
& \frac{\partial d S}{\partial t_{0}}=d \Omega_{0}, \\
& \frac{\partial d S}{\partial t_{k}}=d \Omega_{k}, \quad k \geqslant 1,
\end{aligned}
$$

которые “двойственны” по отношению к первым формулам из (4.9), (4.11) и (4.12), а производные по модулям берутся при постоянной локальной координате $\xi$ (что отвечает выбору некоторой связности на пространстве модулей $\Sigma$ ). Для рациональной 
кривой $\Sigma_{0}$ с единственной отмеченной точкой $P_{0}$ переменные (4.9) и (4.11) отсутствуют, а формулы (4.12) определяют тау-функцию бездисперсионной иерархии КП. В этом случае сингулярная в точке $P_{0}$ часть $\xi_{+}^{n}$ является полиномом $n$-й степени по некоторой глобальной униформизующей координате $\lambda$.

4.3. Препотенциал в многомерном случае. Квазиклассическую тау-функцию (4.9) можно рассматривать как частный случай так называемых препотенциалов комплексных многообразий, которые существуют и для высших комплексных размерностей, как, например, в случае трехмерных многообразий Калаби-Яо, являющихся одним из наиболее интересных примеров с точки зрения теории струн. Общее определение препотенциала включает:

1) комплексное многообразие $\Sigma$, базис в гомологиях с симплектической структурой $A_{\alpha} \circ B_{\beta}=\delta_{\alpha \beta}$, пространство модулей $\mathcal{M}$ комплексных структур на $\Sigma$;

2) производящую дифференциальную форму $\Omega$;

3) набор переменных, а именно периодов $S_{\alpha}=\oint_{A_{\alpha}} \Omega$;

4) дуальные переменные - дуальные периоды $\Pi_{\alpha}=\oint_{B_{\alpha}} \Omega$;

$5)$ условие интегрируемости $\partial \Pi_{\alpha} / \partial S_{\beta}=\partial \Pi_{\beta} / \partial S_{\alpha}$, следующее из билинейных соотношений $\int_{\Sigma} \delta \Omega \wedge \delta \Omega=0$.

Если все эти условия выполнены, как в одномерном случае, рассмотренном в п. 4.2, можно утверждать, что существует препотенциал $\mathcal{F}: \Pi_{\alpha}=\partial \mathcal{F} / \partial S_{\alpha}$. Как и в одномерном случае, все определено только локально на пространстве Тейхмюллера, но на пространстве модулей комплексных структур конструкция согласована с преобразованиями дуальности: $A \leftrightarrow B, S \leftrightarrow \Pi$, в смысле преобразования Лежандра препотенциала

$$
\mathcal{F} \leftrightarrow \mathcal{F}+\sum_{\alpha} S_{\alpha} \Pi_{\alpha}
$$

или, в общей ситуации, полной “электромагнитной” дуальности с группой $S p(2 h, \mathbb{Z})$, где $2 h$ - размерность группы гомологий [24].

Однако существует несколько важных различий, которые необходимо прокомментировать. Пространство модулей для случая трехмерных многообразий Калаби-Яо имеет размерность $h=\operatorname{dim} \mathcal{M}=h^{(2,1)}+1=$ \#(А-циклы), совпадающую с числом $\mathbf{A}$-циклов, в отличие от комплексных кривых, у которых $\operatorname{dim} \mathcal{M}=3 g-3$, в то время как \#(А-циклы $)=g$, и наблюдается противоречие в наивном подсчете. Поэтому в последнем случае необходимо ограничить семейство кривых $\Sigma$ на некоторое подпространство пространства модулей. Инвариантный способ это сделать, как отмечалось выше, заключается в том, чтобы ввести пару мероморфных 1-форм с фиксированными периодами, т.е. если на $\Sigma$

$$
\exists \Omega_{1,2}=\{d \lambda, d z\}: \quad \oint \Omega_{1,2}=\text { const },
$$

то остается в точности $g$ модулей из $3 g-3$, что является наиболее грубой характеристикой интегрируемой системы (см. сноску 5). Однако для комплексных многообразий высших размерностей число циклов, как правило, превышает количество модулей.

Для производящих дифференциальных форм имеется следующая картина: 
а) для трехмерных многообразий Калаби-Яо производящая дифференциальная форма $\Omega \in H^{(3,0)}$ является единственной (с точностью до умножения на число) голоморфной $(3,0)$-формой, и равенство $\int_{\Sigma} \delta \Omega \wedge \delta \Omega=0$ следует из разложения $\delta \Omega \in$ $H^{(3,0)} \oplus H^{(2,1)}$;

б) для комплексных кривых дифференциальная форма $\Omega=d S$ представляет собой мероморфную $(1,0)$-форму, у которой могут быть дополнительные нетривиальные вычеты $\operatorname{res}(d S), \operatorname{res}\left(\xi^{-k} d S\right)$, играющие роль обобщенных периодов (4.11), (4.12).

Таким образом, в последнем случае восстанавливается картина п. 4.2.

4.4. Тау-функция одноматричной модели. Вернемся теперь к изучению свободной энергии одноматричной модели, пользуясь введенными выше новыми понятиями ${ }^{6)}$. Функции $G$ и $y$ (см. (3.7) и (3.10)) определены на двойном накрытии комплексной $x$-плоскости, и на каждом листе они имеют скачки вдоль сегментов или разрезов, пропорциональные плотности собственных значений $\rho(x)$, обращающейся в нуль вне разрезов (см. рис. 1). Поэтому распределение собственных значений (3.12) может быть описано в терминах периодов производящего дифференциала (4.8):

$$
S_{\alpha}=\oint_{A_{\alpha}} d S
$$

где интегралы вычисляются по контурам, окружающим носители собственных значений $C_{\alpha}$, которые могут быть идентифицированы (за исключением одного из них, т.е. $k=1,2, \ldots, n-1)$ с набором канонических А-циклов на кривой (3.10) (см. рис. 1). Производящий дифференциал с резольвентой (3.4) одноматричной модели может быть эквивалентно выбран в виде

$$
d S=\frac{i}{4 \pi} y d x
$$

где теперь $x$ и $y$ являются координатами на кривой $\Sigma$, определяемой уравнением (3.10), поскольку (см. (3.9) $)^{7)}$

$$
\oint G d x=-\frac{1}{2} \oint y d x
$$

Как $x$, так и $y$ являются глобально определенными мероморфными функциями на кривой (3.10), поэтому $\oint d x=0$ и $\oint d y=0$ для любого выбора замкнутых контуров. Соотношения (4.14) естественно выполняются для дифференциала (4.19), когда производные вычисляются при фиксированных вычетах и коэффициентах $\left\{t_{k}\right\}$ потенциала (1.4).

Важным фактом является то, что лагранжевы множители (см. (3.13)) могут быть также переписаны как интегралы периодов того же самого производящего дифференциала (4.19), но уже по двойственным В-контурам (см. рис. 1)

$$
\Pi_{\alpha}=-W\left(x_{\alpha}^{*}\right)+2 \int_{\mathbf{C}} d x \rho(x) \ln \left(x-x_{\alpha}^{*}\right),
$$

6) Геометрическая формулировка для свободной энергии матричной модели была впервые введена в работе [18], а также обсуждалась позже в [19], [20], [25].

7) Вопрос о вычетах (4.11), (4.12) требует аккуратности. Для симметричного выбора (4.19) эти вычеты могут вычисляться в любой из бесконечностей $x=\infty$, в то время как в асимметричном случае (3.4) их следует вычислять только на нефизическом листе, где резольвента (3.4) сингулярна. 


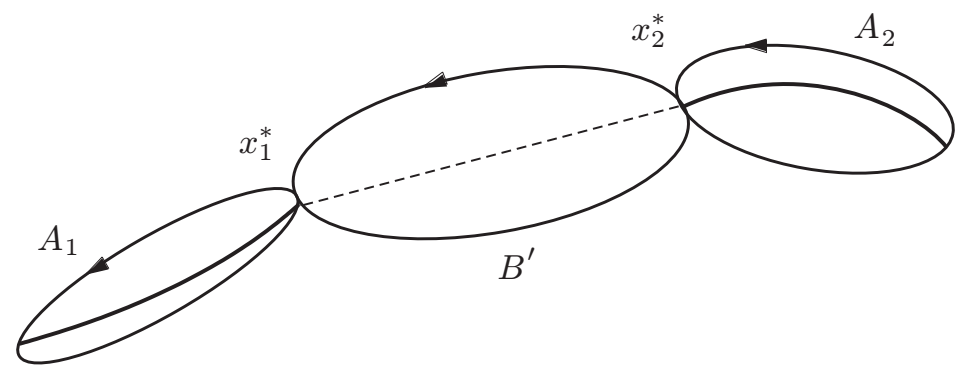

Рис. 5. Вычисление интеграла в формуле (4.22). Интегралы по разрезам $C_{k}$ при $k=1,2$ (жирные линии), окруженным $A_{k}$-циклами, заменяются на интеграл по циклу $B^{\prime}$, охватывающему логарифмический разрез (штриховая линия). Последний же трансформируется в интеграл вдоль контура, соединяющего точки $x_{1}^{*}$ и $x_{2}^{*}$ (но уже без логарифма в подынтегральном выражении), который есть половина периода, вычисленного по В-циклу, проходящему в одну сторону между разрезами $C_{1}$ и $C_{2}$ по одному из листов, а в обратную сторону - по другому.

где точки $x_{\alpha}^{*} \in C_{\alpha}$ могут быть выбраны из соображений удобства, причем каждая на $\alpha$-й части носителя; при этом можно “забыть” про модуль (см. сноску 3 ) и величины $\Pi_{\alpha}$ становятся голоморфными объектами.

Интеграл в формуле (4.20) может быть легко трансформирован в контурный интеграл (для простоты мы явно выпишем процедуру для случая двух разрезов (см. рис. 2), поскольку ее обобщение очевидно). В этом случае существует два числа заполнения $S_{1}+S_{2}=t_{0}$ и более удобно взять в качестве независимых переменных $t_{0}$ и $S=S_{2}$. Тогда

$$
\sum_{\alpha} \Pi_{\alpha} S_{\alpha}=\left(\Pi_{2}-\Pi_{1}\right) S+\Pi_{1} t_{0}
$$

и вариация свободной энергии (3.11) по $S$ дает

$$
\frac{\partial \mathcal{F}}{\partial S}=W\left(x_{1}^{*}\right)-W\left(x_{2}^{*}\right)+2 \sum_{\alpha=1,2} \int_{C_{\alpha}} d x \rho(x) \ln \frac{x-x_{2}^{*}}{x-x_{1}^{*}} .
$$

Поскольку для интеграла по контуру, охватывающему полный носитель, имеем

$$
\oint_{\mathbf{C}} d x G(x) \ln \frac{x-x_{1}^{*}}{x-x_{2}^{*}}=0
$$

(такой контур может быть деформирован к охватывающему точку $x=\infty$, в которой у подынтегрального выражения нет вычета), сумма в формуле (4.21) может быть приведена к виду (см. рис. 5)

$$
\begin{gathered}
\sum_{\alpha=1,2} \int_{C_{\alpha}} d x \rho(x) \ln \frac{x-x_{1}^{*}}{x-x_{2}^{*}}=\sum_{\alpha=1,2} \frac{1}{2 \pi i} \oint_{C_{\alpha}} d x G(x) \ln \frac{x-x_{1}^{*}}{x-x_{2}^{*}}= \\
=-\frac{1}{2 \pi i} \oint_{B^{\prime}} d x G(x) \ln \frac{x-x_{1}^{*}}{x-x_{2}^{*}}=\int_{x_{2}^{*}}^{x_{1}^{*}} G(x) d x .
\end{gathered}
$$


В итоге это приводит к тому, что производная (4.21) выражается формулой

$$
\frac{\partial \mathcal{F}}{\partial S}=W\left(x_{1}^{*}\right)-W\left(x_{2}^{*}\right)-2 \int_{x_{2}^{*}}^{x_{1}^{*}} G(x) d x=\int_{x_{2}^{*}}^{x_{1}^{*}} y d x=-\frac{1}{2} \oint_{B} y d x=2 \pi i \oint_{B} d S,
$$

в которой контур $B$ проходит от $x_{1}^{*}$ к $x_{2}^{*}$ и назад по другому листу кривой (не путать с контуром $B^{\prime}$, охватывающим точки $x_{1}^{*}$ и $x_{2}^{*}$ !). Для произвольного числа разрезов следует аналогично подставить

$$
\sum_{\alpha=1}^{n} \Pi_{\alpha} S_{\alpha}=\left(\Pi_{1}-\Pi_{n}\right) S_{1}+\cdots+\left(\Pi_{n-1}-\Pi_{n}\right) S_{n-1}+\Pi_{n} t_{0}
$$

и проделать тот же трюк с логарифмическими разрезами. Эта процедура приводит к отождествлению свободной энергии матричной модели с препотенциалом (4.9).

$\mathrm{K}$ набору параметров (4.18) следует добавить полное число собственных значений $(3.5)^{8)}$

$$
t_{0}=\left.\frac{1}{2 \pi i} \operatorname{res}\right|_{\infty}(d S)=\frac{2 f_{n-1}}{(n+1) t_{n+1}},
$$

которое в точности совпадает с нулевым временем (4.11), и параметры потенциала (1.4), переписывающиеся в виде времен (4.12), т.е.

$$
t_{k}=\left.\frac{1}{2 \pi i k} \operatorname{res}\right|_{\infty}\left(x^{-k} d S\right), \quad k=1,2, \ldots, n,
$$

поскольку $x$ играет роль обратной локальной координаты в точке $x=\infty$. При этом имеем явно

$$
d \Omega_{0}=\left.\frac{\partial d S}{\partial t_{0}}\right|_{\mathbf{S}, \mathbf{t}}=(n+1) t_{n+1} \frac{x^{n-1} d x}{y}+2 \sum_{k=0}^{n-2} \frac{\partial f_{k}}{\partial t_{0}} \frac{x^{k} d x}{y},
$$

а зависимость коэффициентов $\left\{f_{k}\right\}, k=0,1, \ldots, n-2$, от $t_{0}$ фиксируется периодами

$$
\oint_{A_{\alpha}}\left((n+1) t_{n+1} \frac{x^{n-1} d x}{y}+2 \sum_{k=0}^{n-2} \frac{\partial f_{k}}{\partial t_{0}} \frac{x^{k} d x}{y}\right)=0
$$

которые при $\alpha=1,2, \ldots, n-1$ представляют собой в точности $n-1$ соотношений на производные от $f_{0}, f_{1}, \ldots, f_{n-2}$ по $t_{0}$.

Для производных по параметрам потенциала (4.25) получим дифференциалы

$$
d \Omega_{k}=\left.\frac{\partial d S}{\partial t_{k}}\right|_{\mathbf{S}, t_{0}}=\frac{W^{\prime}(x) k x^{k-1} d x}{y}+2 \sum_{j=0}^{n-2} \frac{\partial f_{j}}{\partial t_{k}} \frac{x^{k} d x}{y},
$$

удовлетворяющие аналогично

$$
\oint_{A_{\alpha}} d \Omega_{k}=\oint_{A_{\alpha}} \frac{W^{\prime}(x) k x^{k-1} d x}{y}+2 \sum_{j=0}^{n-2} \frac{\partial f_{j}}{\partial t_{k}} \oint_{A_{\alpha}} \frac{x^{k} d x}{y}=0
$$

${ }^{8)}$ Под точкой $\infty$ в дальнейшем для краткости будем понимать точку $\infty+$ или $x=\infty$ на верхнем листе гиперэллиптической римановой поверхности (3.10), отвечающем положительному выбору знака квадратного корня, т.е. когда $y=+\sqrt{W^{\prime}(x)^{2}+4 f(x)}$. 
и это опять приводит к системе линейных уравнений, разрешающихся относительно $\partial f_{j} / \partial t_{k}$. Для полноты картины к (4.20) следует добавить выражение

$$
\frac{\partial \mathcal{F}}{\partial t_{k}}=\left.\frac{1}{2 \pi i} \operatorname{res}\right|_{\infty}\left(x^{k} d S\right), \quad k>0,
$$

а также формулу ${ }^{9)}$

$$
\frac{\partial \mathcal{F}}{\partial S_{n}}=\Pi_{n}=\int_{B_{n}^{\prime}} d S
$$

с правильно подобранным контуром $B_{n}^{\prime}$, проходящим из $\infty_{-}$на нижнем листе к $\infty_{+}$ на верхнем листе через $n$-й разрез (напомним, что вместо $t_{0}$ можно эквивалентно использовать параметр $\left.S_{n}=t_{0}-\sum_{i=1}^{n-1} S_{\alpha}\right)$. Мы видим, что в случае дифференциала (4.19) одноматричной модели эти определения требуют корректной доработки, однако, например, в контексте геометрии АдС/КТП-соответствия [27] возникает пример производящего дифференциала с регулярным поведением в точках $\infty_{ \pm}$, так что формулы типа (4.31) можно применять буквально.

4.5. Формула вычетов. Перейдем теперь к обсуждению одной из наиболее универсальных и красивых формул для квазиклассической тау-функции, а именно формулы вычетов для ее третьих производных

$$
\frac{\partial^{3} \mathcal{F}}{\partial T_{I} \partial T_{J} \partial T_{K}}=\left.\frac{1}{2 \pi i} \operatorname{res}\right|_{d x=0}\left(\frac{d H_{I} d H_{J} d H_{K}}{d x d y}\right),
$$

универсальной для зависимости функции $\mathcal{F}$ от любых переменных $\left\{T_{I}\right\}$, в которой $\left\{d H_{I}\right\}$ являются соответствующими им дифференциалами. Существование простой формулы (4.32) для третьих производных вместе с отсутствием подобных выражений для старших производных определенно имеет струнную природу, связанную с формулировкой теории на мировом листе, в которой трехточечные корреляционные функции на сфере играют выделенную роль.

4.5.1. Голоморфные дифференциалы. Выведем формулы для третьих производных препотенциала $\mathcal{F}$, следуя способу, предложенному Кричевером [22] и явно примененному в работе [26]. Заметим сначала, что производные элементов $T_{i j}$ матрицы периодов (4.10) могут быть выражены через интеграл по границе $\partial \Sigma$ разрезанной римановой поверхности $\Sigma$ :

$$
\frac{\partial T_{\alpha \beta}}{\partial S_{\gamma}} \equiv \partial_{\gamma} T_{\alpha \beta}=\int_{B_{\beta}} \partial_{\gamma} d \omega_{\alpha}=-\int_{\partial \Sigma} \omega_{\beta} \partial_{\gamma} d \omega_{\alpha},
$$

9) Наивно понимаемый интеграл в (4.31) расходится, и его следует доопределить подходящей регуляризацией. Это можно просто проигнорировать, если требуются только формулы вычетов для третьих производных (к рассмотрению которых мы перейдем в п. 4.5), например для решения уравнений Виттена-Дийкграафа-Верлинде-Верлинде [26]. Простейший способ обойти эти сложности - рассматривать пару отмеченных точек $\infty$ и $\infty_{-}$как вырожденную "ручку". Тогда вычет (4.24) является вырождением дополнительного А-периода, в то время как интеграл (4.31) наследуется из вырождения дополнительного В-периода (см. рис. 1). С практической точки зрения этот расходящийся период всегда может быть заменен конечной величиной (см., например, гауссов пример (3.19)). 
где $\omega_{\beta}=\int^{P} d \omega_{\beta}$ представляют собой абелевы интегралы, значения которых на двух копиях цикла на разрезанной римановой поверхности (см. рис. 4) обозначены ниже как $\omega_{\beta}^{ \pm}$. Действительно, вычисление интеграла в правой части (4.33) приводит к следующему результату:

$$
\begin{aligned}
\int_{\partial \Sigma} \omega_{\beta} \partial_{\gamma} d \omega_{\alpha}=\sum_{\rho}\left(\int_{B_{\rho}} \omega_{\beta}^{+} \partial_{\gamma} d \omega_{\alpha}-\int_{B_{\rho}} \omega_{\beta}^{-} \partial_{\gamma} d \omega_{\alpha}\right)- \\
-\sum_{\rho}\left(\int_{A_{\rho}} \omega_{\beta}^{+} \partial_{k} d \omega_{\alpha}-\int_{A_{\rho}} \omega_{\beta}^{-} \partial_{\gamma} d \omega_{\alpha}\right)= \\
=\sum_{\rho} \oint_{B_{\rho}}\left(\oint_{A_{\rho}} d \omega_{\beta}\right) \partial_{\gamma} d \omega_{\alpha}-\sum_{\rho} \oint_{A_{\rho}}\left(\oint_{B_{\rho}} d \omega_{\beta}\right) \partial_{\gamma} d \omega_{\alpha}= \\
=\sum_{\rho}\left(\oint_{A_{\rho}} d \omega_{\beta}\right) \oint_{B_{\rho}} \partial_{\gamma} d \omega_{\alpha}-\sum_{\rho}\left(\oint_{B_{\rho}} d \omega_{\beta}\right) \oint_{A_{\rho}} \partial_{\gamma} d \omega_{\alpha}=-\partial_{\gamma} T_{\alpha \beta}
\end{aligned}
$$

где мы воспользовались формулой (4.1). Теперь можно переписать формулу (4.33) в виде

$$
\partial_{\gamma} T_{\alpha \beta}=-\int_{\partial \Sigma} \omega_{\beta} \partial_{\gamma} d \omega_{\alpha}=\int_{\partial \Sigma} \partial_{\gamma} \omega_{\beta} d \omega_{\alpha}=\left.\sum \operatorname{res}\right|_{d \lambda=0}\left(\partial_{\gamma} \omega_{\beta} d \omega_{\alpha}\right),
$$

где суммирование производится по всем вычетам подынтегрального выражения, т.е. по всем вычетам $\partial_{\gamma} \omega_{\beta}$, так как дифференциалы $d \omega_{\alpha}$ голоморфны. Чтобы исследовать эти сингулярности и прояснить последнее равенство в (4.35), мы сначала обсудим точный смысл производных $\partial_{\gamma}$ по модулям, или введем соответствующую связность на пространстве модулей $\Sigma$.

Для этого введем ковариантно-постоянную функцию модулей, скажем абелев интеграл $x=\int^{P} d x$, т.е. выберем такую связность на пространстве модулей, при которой $\partial_{\gamma} x=0$. Роль ковариантно-постоянной функции локально может играть любая из координат, в простейшем возможном описании кривой одним уравнением на две комплексные переменные это может быть любая из функций, входящих в уравнение $F(x, y)=0$. Тогда, используя это уравнение, можно выразить координату у как функцию $x$ и модулей (коэффициентов уравнения). Любой абелев интеграл $\omega_{\beta}$ может быть при этом выражен в терминах переменной $x$, и в окрестности критических точек $\left\{x_{a}\right\}$, где $d x=0$ (в случае общего положения), мы получим разложение

$$
\omega_{\beta}(x) \underset{x \rightarrow x_{\alpha}}{=} \omega_{\beta a}+c_{\beta a} \sqrt{x-x_{a}}+\cdots,
$$

производные которого

$$
\left.\partial_{\gamma} \omega_{\beta} \equiv \partial_{\gamma} \omega_{\beta}\right|_{x=\mathrm{const}}=-\frac{c_{\beta a}}{2 \sqrt{x-x_{a}}} \partial_{\gamma} x_{a}+\text { regular }
$$

где через "regular" мы обозначили несингулярные члены, обладают полюсами первого порядка в точках $x=x_{a}$, и мы выписали их с точностью до регулярных членов, которые не дают вкладов в формулу (4.35). Точный коэффициент в (4.37) можно 
вычислить, связав функцию $x$ с производящим дифференциалом $d S=y d x$. Используя соотношение

$$
y(x) \underset{x \rightarrow x_{a}}{=} \Gamma_{a} \sqrt{x-x_{a}}+\cdots,
$$

где $\Gamma_{a}=\sqrt{\prod_{b \neq a}\left(x_{a}-x_{b}\right)}$, или

$$
\frac{\partial}{\partial S_{\gamma}} y(x)=-\frac{\Gamma_{a}}{2 \sqrt{x-x_{a}}} \frac{\partial x_{a}}{\partial S_{\gamma}}+\text { regular }
$$

вместе с

$$
d y=\frac{\Gamma_{a}}{2 \sqrt{x-x_{a}}} d x+\text { regular }
$$

И

$$
d \omega_{\beta}=\frac{c_{\beta a}}{2 \sqrt{x-x_{a}}} d x+\cdots,
$$

а также следующее из (4.19) и (4.14) разложение

$$
d \omega_{\gamma}=\partial_{\gamma} d S=-\frac{\Gamma_{a} \partial_{\gamma} x_{a}}{2 \sqrt{x-x_{a}}} d x+\text { regular }
$$

окончательно получаем

$$
\begin{aligned}
\operatorname{res}\left(\partial_{\gamma} \omega_{\beta} d \omega_{\alpha}\right) & =\sum_{a} \operatorname{res}\left(\frac{c_{\beta a} \partial_{\gamma} x_{a}}{2 \sqrt{x-x_{a}}} d \omega_{\alpha}\right)=\sum_{a} \operatorname{res}\left(\frac{d \omega_{\beta}}{d x} d \omega_{\alpha} \partial_{\gamma} x_{a}\right)= \\
& =\sum_{a} \operatorname{res}\left(\frac{d \omega_{\alpha} d \omega_{\beta} d \omega_{\gamma}}{d x d y}\right) .
\end{aligned}
$$

В случае гиперэллиптических кривых (3.10) для одноматричной модели для вывода формулы вычетов можно использовать формулу [23]

$$
\frac{\partial T_{\alpha \beta}}{\partial x_{a}}=\widehat{\omega}_{\alpha}\left(x_{a}\right) \widehat{\omega}_{\beta}\left(x_{a}\right),
$$

где $\widehat{\omega}_{\alpha}\left(x_{a}\right)=d \omega_{\alpha}(x) /\left.d \sqrt{x-x_{a}}\right|_{x=x_{a}}$ - "значение" канонического дифференциала в критической точке.

4.5.2. Мероморфные дифференциалы. Практически без изменений вывод формулы вычетов может быть проведен в случае мероморфных дифференциалов. ИмеeM

поэтому

$$
\frac{\partial \mathcal{F}}{\partial t_{k}}=\left.\frac{1}{2 \pi i} \operatorname{res}\right|_{\infty}\left(x^{k} d S\right), \quad k>0,
$$

$$
\frac{\partial^{2} \mathcal{F}}{\partial t_{k} \partial t_{n}}=\left.\frac{1}{2 \pi i} \operatorname{res}\right|_{\infty}\left(x^{k} d \Omega_{n}\right)=\left.\frac{1}{2 \pi i} \operatorname{res}\right|_{\infty}\left(\left(\Omega_{k}\right)_{+} d \Omega_{n}\right),
$$

где $\left(\Omega_{k}\right)_{+}$является сингулярной частью проинтегрированной 1-формы $d \Omega_{k}$. Далее имеем

$$
\begin{aligned}
\left.\frac{\partial}{\partial t_{m}} \operatorname{res}\right|_{\infty}\left(x^{k} d \Omega_{n}\right) & =\left.\operatorname{res}\right|_{\infty}\left(x^{k} \frac{\partial d \Omega_{n}}{\partial t_{m}}\right)=-\left.\operatorname{res}\right|_{\infty}\left(\left(d \Omega_{k}\right)_{+} \frac{\partial \Omega_{n}}{\partial t_{m}}\right)= \\
& =-\left.\operatorname{res}\right|_{\infty}\left(d \Omega_{k} \frac{\partial \Omega_{n}}{\partial t_{m}}\right) .
\end{aligned}
$$

2 Теоретическая и математическая физика, т. 147, № 2, 2006 г. 
Последнее выражение может быть переписано в виде

$$
\begin{aligned}
-\left.\operatorname{res}\right|_{\infty}\left(d \Omega_{k} \frac{\partial \Omega_{n}}{\partial t_{m}}\right) & =\oint_{\partial \Sigma}\left(d \Omega_{k} \frac{\partial \Omega_{n}}{\partial t_{m}}\right)+\left.\sum_{a} \operatorname{res}\right|_{x_{a}}\left(d \Omega_{k} \frac{\partial \Omega_{n}}{\partial t_{m}}\right)= \\
& =\left.\sum_{a} \operatorname{res}\right|_{x_{a}}\left(d \Omega_{k} \frac{\partial \Omega_{n}}{\partial t_{m}}\right)
\end{aligned}
$$

поскольку согласно (4.29) $\oint_{\partial \Sigma}\left(\partial \Omega_{n} / \partial t_{m}\right) d \Omega_{k}=0$ (ср. с (4.34)):

$$
\begin{aligned}
\int_{\partial \Sigma} \Omega_{j} \frac{\partial}{\partial t_{k}} d \Omega_{i} & =\sum_{\alpha}\left(\int_{B_{\alpha}} \Omega_{j}^{+} \frac{\partial}{\partial t_{k}} d \Omega_{i}-\int_{B_{\alpha}} \Omega_{j}^{-} \frac{\partial}{\partial t_{k}} d \Omega_{i}\right)- \\
& -\sum_{\alpha}\left(\int_{A_{\alpha}} \Omega_{j}^{+} \frac{\partial}{\partial t_{k}} d \Omega_{i}-\int_{A_{\alpha}} \Omega_{j}^{-} \frac{\partial}{\partial t_{k}} d \Omega_{i}\right)= \\
= & \sum_{\alpha} \oint_{B_{\alpha}}\left(\oint_{A_{\alpha}} d \Omega_{j}\right) \frac{\partial}{\partial t_{k}} d \Omega_{i}-\sum_{\alpha} \oint_{A_{\alpha}}\left(\oint_{B_{\alpha}} d \Omega_{j}\right) \frac{\partial}{\partial t_{k}} d \Omega_{i}= \\
= & \sum_{\alpha}\left(\oint_{A_{\alpha}} d \Omega_{j}\right) \oint_{B_{\alpha}} \frac{\partial}{\partial t_{k}} d \Omega_{i}-\sum_{\alpha}\left(\oint_{B_{\alpha}} d \Omega_{j}\right) \oint_{A_{\alpha}} \frac{\partial}{\partial t_{k}} d \Omega_{i}=0
\end{aligned}
$$

Теперь, как и в голоморфном случае, рассмотрим первые члены разложения

$$
\Omega_{n}(x) \underset{x \rightarrow x_{a}}{=} \Omega_{n a}+\gamma_{n a} \sqrt{x-x_{a}}+\cdots,
$$

откуда следует, что

$$
\left.\frac{\partial}{\partial t_{k}} \Omega_{j} \equiv \frac{\partial}{\partial t_{k}} \Omega_{j}\right|_{x=\text { const }}=-\frac{\gamma_{j a}}{2 \sqrt{x-x_{a}}} \frac{\partial x_{a}}{\partial t_{k}}+\text { regular } .
$$

Тогда, используя (4.38)-(4.40) и формулу

$$
d \Omega_{j}=\frac{\gamma_{j a}}{2 \sqrt{x-x_{a}}} d x+\cdots
$$

а также соотношение, следующее из (4.16), (4.39):

$$
d \Omega_{k}=\frac{\partial}{\partial t_{k}} d S=-\frac{\Gamma_{a} d x}{2 \sqrt{x-x_{a}}} \frac{\partial x_{a}}{\partial t_{k}}+\text { regular }
$$

для (4.48) получим

$$
\begin{gathered}
\frac{\partial^{3} \mathcal{F}}{\partial t_{k} \partial t_{n} \partial t_{m}}=\left.\frac{1}{2 \pi i} \sum_{a} \operatorname{res}\right|_{x_{a}}\left(d \Omega_{k} \frac{\partial \Omega_{n}}{\partial t_{m}}\right)=-\left.\frac{1}{2 \pi i} \sum_{a} \operatorname{res}\right|_{x_{a}}\left(d \Omega_{k} \frac{\gamma_{n a}}{2 \sqrt{x-x_{a}}} \frac{\partial x_{a}}{\partial t_{m}}\right)= \\
=-\left.\frac{1}{2 \pi i} \sum_{a} \operatorname{res}\right|_{x_{a}}\left(d \Omega_{k} \frac{d \Omega_{n}}{d x} \frac{\partial x_{a}}{\partial t_{m}}\right)=\left.\frac{1}{2 \pi i} \sum_{a} \operatorname{res}\right|_{x_{a}}\left(\frac{d \Omega_{k} d \Omega_{n} d \Omega_{m}}{d x d y}\right) .
\end{gathered}
$$

Вывод формулы вычетов для набора переменных, включающих время $t_{0}$, отвечающее абелеву дифференциалу третьего рода (4.26), может быть получен абсолютно 
так же, и аналогичным образом доказывается формула вычетов для смешанных производных. Таким образом, мы получаем окончательно

$$
\begin{aligned}
\frac{\partial^{3} \mathcal{F}}{\partial T_{I} \partial T_{J} \partial T_{K}} & =\left.\frac{1}{2 \pi i} \sum_{a} \operatorname{res}\right|_{x_{a}}\left(\frac{d H_{I} d H_{J} d H_{K}}{d x d y}\right)= \\
& =\left.\frac{1}{2 \pi i} \sum_{a} \operatorname{res}\right|_{x_{a}}\left(\frac{\phi_{I} \phi_{J} \phi_{K}}{d x / d y} d y\right)=\sum_{a} \Gamma_{a}^{2} \phi_{I}\left(x_{a}\right) \phi_{J}\left(x_{a}\right) \phi_{K}\left(x_{a}\right)= \\
& =\sum_{a} \frac{\widehat{H}_{I}\left(x_{a}\right) \widehat{H}_{J}\left(x_{a}\right) \widehat{H}_{K}\left(x_{a}\right)}{\prod_{b \neq a}\left(x_{a}-x_{b}\right)^{2}}
\end{aligned}
$$

для полного набора переменных $\left\{T_{I}\right\}=\left\{t_{k}, t_{0}, S_{i}\right\}$ и соответствующих им 1-форм $\left\{d H_{I}\right\}=\left\{d \Omega_{k}, d \Omega_{0}, d \omega_{i}\right\}$. В формуле (4.55) введены мероморфные функции

$$
\phi_{I}(x)=\frac{d H_{I}}{d y}=\frac{\widehat{H}_{I}(x)}{R^{\prime}(x)}
$$

для любого (мероморфного или голоморфного) дифференциала на гиперэллиптической кривой $(3.10) d H_{I}=\widehat{H}_{I}(x) d x / y$.

\section{5. КОМПЛЕКСНАЯ КРИВАЯ ДВУХМАТРИЧНОЙ МОДЕЛИ}

Перейдем теперь к случаю двухматричной модели (2.5). Так же, как и для одноматричного случая, мы сначала рассмотрим вспомогательное комплексное многообразие $\Sigma$, а затем уже перейдем к обсуждению квазиклассической тау-функции.

5.1. Квазиклассическое приближение для двухматричной модели. Рассмотрим свободную энергию двухматричной модели (2.4), (2.5) в планарном пределе. Подобно одноматричному случаю, в планарном или квазиклассическом пределе можно заменить прямое вычисление интеграла по собственным значаниям (2.5) решением соответствующей вариационной задачи. По крайней мере, для вещественной задачи в двухматричной модели со взаимно комплексно-сопряженными собственными значениями ${ }^{10)}$ анализ упрощается из-за основного свойства комплексного логарифма

$$
\Delta \ln \left|z-z^{\prime}\right|=2 \pi \delta^{(2)}\left(z-z^{\prime}\right)
$$

т.е. двумерное кулоновское отталкивание комплексно-сопряженных собственных значений определяется ядром оператора, обратного двумерному оператору Лапласа $\Delta=4 \partial_{z} \partial_{\bar{z}}$. Поэтому для потенциала (2.4) плотность собственных значений является некоторой константой внутри носителя или (двумерной) области собственных

10) Иногда такая модель довольно неудачно называется моделью "нормальных матриц". Следует подчеркнуть, что при изучении функции распределения (2.5) с конкретно выбранным "кроссчленом" $\operatorname{Tr}\left(\Phi^{\dagger} \Phi\right)$ в потенциале (2.4) единственным существенным моментом является выбор вещественного сечения или конкретного класса контуров интегрирования по каждой переменной $z_{i}$ и $\bar{z}_{i}$. Различие же между "нормальной" (с коммутирующими матрицами $\Phi$ и $\Phi^{\dagger}$ ) и обычной двухматричной моделями возникает только при изучении многоследовых операторов, которое выходит за рамки геометрической картины, обсуждаемой в данной работе. 


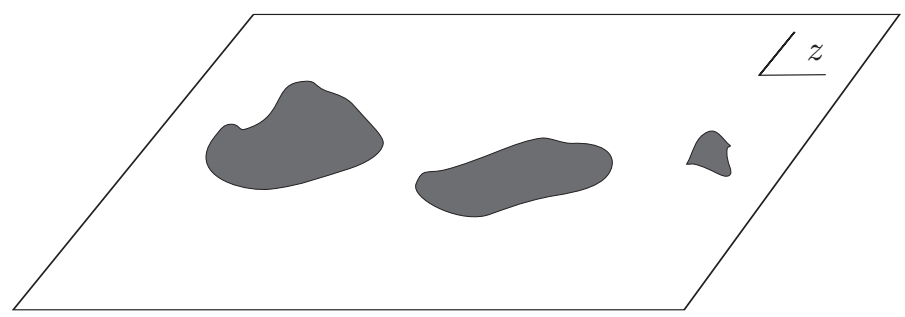

Рис. 6. Капли в плоскости собственных значений вещественной задачи для двухматричной модели.

значений и равна нулю вне ее, т.е.

$$
\rho(z, \bar{z}) \propto \begin{cases}1, & z \in \mathrm{D} \\ 0, & z \notin \mathrm{D}\end{cases}
$$

а соответствующая вещественная задача сводится к нахождению потенциальной энергии некоторой области D (в общем случае набора капель) в комплексной zплоскости, наполненной заряженной жидкостью с постоянной плотностью заряда $(\text { см. рис. } 6)^{11)}$. В отличие от одномерного случая, в данной ситуации плотность собственных значений (5.2) постоянна, а нетривиальная информация об их распределении содержится в площади и форме области, выражаемой через коэффициенты потенциала матричной модели (2.4) (точнее, его гармонической части).

Уравнение стационарности

$$
\bar{z}_{i}=W^{\prime}\left(z_{i}\right)-\hbar \sum_{j \neq i} \frac{1}{z_{i}-z_{j}},
$$

следующее из функционала свободной энергии, отвечающего эффективному потенциалу

$$
V_{\mathrm{eff}}(z, \bar{z})=\sum_{i}\left(\bar{z}_{i} z_{i}-W\left(z_{i}\right)-\widetilde{W}\left(\bar{z}_{i}\right)\right)+\hbar \sum_{i<j} \ln \left|z_{i}-z_{j}\right|^{2},
$$

для двухматричного случая (2.5) может быть переписано в виде

$$
\bar{z}=W^{\prime}(z)-G(z)
$$

(вместе с комплексно-сопряженной формулой) после введения, как и в одноматричном случае $(3.3),(3.4)$, плотности $\rho(z, \bar{z})$ или функции

$$
G(z)=\hbar\left\langle\operatorname{Tr} \frac{1}{z-\Phi}\right\rangle_{\Phi, \Phi^{\dagger}}=\frac{1}{\pi} \int_{\mathbb{C}} \frac{\rho(\zeta, \bar{\zeta}) d^{2} \zeta}{z-\zeta}=\frac{1}{\pi} \int_{\mathrm{D}} \frac{d^{2} \zeta}{z-\zeta},
$$

где использована формула (5.2), или (голоморфной) резольвенты двухматричной модели. Когда взаимодействие в правой части (5.3) отсутствует (в пределе $\hbar \rightarrow 0)$,

\footnotetext{
11)Эта задача недавно оказалась в центре внимания в контексте так называемого лапласовского роста (см., например, работы [28] и приведенную в них литературу).
} 
резольвента обращается в нуль, и уравнение (5.5) можно записать в симметричном виде:

$$
\left(\bar{z}-W^{\prime}(z)\right)\left(z-\bar{W}^{\prime}(\bar{z})\right)=0 .
$$

В общем случае уравнение (5.7) имеет $n \tilde{n}$ решений - точек в комплексной плоскости $\mathbb{C}$, или, в комплексифицированной ситуации, $(z, \bar{z}) \rightarrow(z, \tilde{z}) \in \mathbb{C}^{2}$ в двумерном комплексном евклидовом пространстве. Уравнение (5.7), как и в одноматричном случае, имеет смысл вырожденной классической кривой. Если имеется кулоновское взаимодействие собственных значений (в выражении (5.4) это буквально кулоновское взаимодействие, что оправдывает терминологию, которую мы применяли формально даже в случае одноматричной модели), этот набор точек превратится в набор одномерных “траекторий”, или в вещественное сечение некоторой комплексной кривой. Ниже мы сначала займемся изучением структуры этой кривой или римановой поверхности в случае общего положения, отвечающего комплексной задаче в двухматричной модели, а затем вернемся к вещественной задаче.

5.2. Уравнение и род кривой в комплексной задаче. Для общих полиномиальных потенциалов $W$ и $\widetilde{W}$ степеней $n+1$ и $\tilde{n}+1$, соответственно, старшие члены аналитического уравнения классической кривой (5.7) имеют вид $z^{n} \tilde{z}^{\tilde{n}}+a_{\tilde{n}} z^{n+1}+$ $\tilde{a}_{n} \bar{z}^{\tilde{n}+1}$ с коэффициентами

$$
a_{\tilde{n}}=-\left((\tilde{n}+1) \tilde{t}_{\tilde{n}+1}\right)^{-1}, \quad \tilde{a}_{n}=-\left((n+1) t_{n+1}\right)^{-1} .
$$

Это означает, что общая десингуляризация классической кривой (5.7) имеет следующий вид [29] (см. также [30]):

$$
\begin{aligned}
F(z, \tilde{z}) & =\left((n+1)(\tilde{n}+1) t_{n+1} \tilde{t}_{\tilde{n}+1}\right)^{-1}\left(\tilde{z}-W_{n}^{\prime}(z)\right)\left(z-\widetilde{W}_{\tilde{n}}^{\prime}(\tilde{z})\right)+\cdots= \\
& =z^{n} \tilde{z}^{\tilde{n}}+a_{\tilde{n}} z^{n+1}+\tilde{a}_{n} \bar{z}^{\tilde{n}+1}+\sum_{(i, j) \in(\text { N.P. })_{+}} f_{i j} z^{i} \tilde{z}^{j}
\end{aligned}
$$

где уже использованы комплексифицированные обозначения $(z, \tilde{z}) \in \mathbb{C}^{2}$ для координат, вкладывающих кривую (5.9) в двумерное комплексное пространство, вместо взаимно комплексно-сопряженных координат $(z, \bar{z}) \in \mathbb{C}$ точки плоскости собственных значений, изображенной на рис. 6. Для дальнейших целей будет удобно уточнить вид уравнения (5.9) для вещественного потенциала (2.4) с комплексносопряженными коэффициентами и одинаковыми степенями $n=\tilde{n}$ :

$$
F(z, \tilde{z})=z^{n} \tilde{z}^{n}+a_{n} z^{n+1}+\tilde{a}_{n} \tilde{z}^{n+1}+\sum_{i, j \in(\text { N.P. })_{+}} f_{i j} z^{i} \tilde{z}^{j}=0 .
$$

Свойства кривых (5.9), (5.10) могут быть легко исследованы с помощью многоугольников Ньютона (см. рис. 7 для случая $n=\tilde{n}$, для произвольных значений $n$ и $\tilde{n}$ квадрат размера $(n+1) \times(n+1)$ следует заменить на прямоугольник со сторонами $(n+1) \times(\tilde{n}+1)$, при этом все остальные элементы конструкции остаются без изменений). Три первых члена в правой части формулы (5.9) отвечают трем точкам на границе многоугольника, а суммирование по (N.P.)+ обозначает сумму по всем 


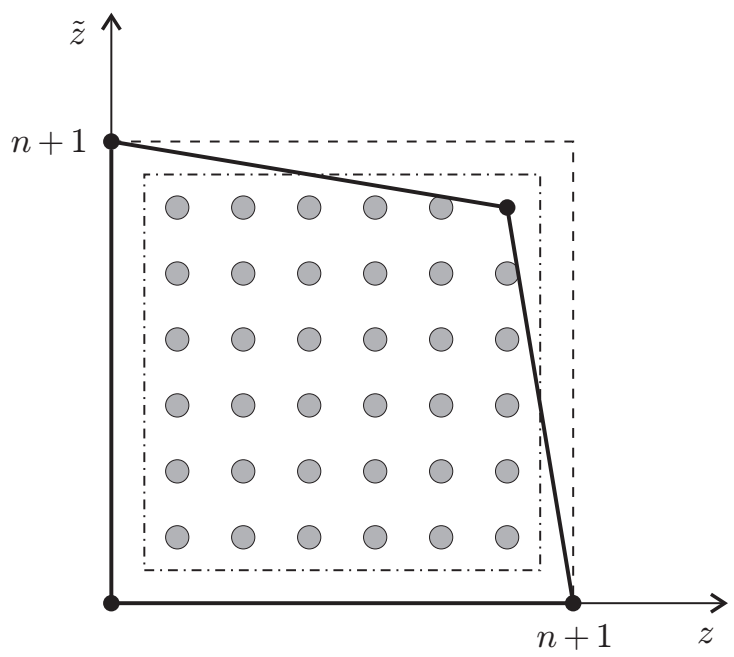

Рис. 7. Многоугольник Ньютона для кривой (5.10). Члены старших степеней в формуле (5.10) определяют форму многоугольника, а число целых точек внутри него определяет количество независимых голоморфных дифференциалов, или род кривой. Очевидно, что это число эквивалентно площади двойственного квадрата без единственной (черной) точки, следовательно, $g=n \tilde{n}-1=n^{2}-1$.

точкам внутри многоугольника (включая точки на обеих осях, не отмеченные на рис. 7).

Простейший базис голоморфных дифференциалов на кривых (5.9), (5.10) может быть выбран в виде

$$
d v_{i j}=z^{i} \tilde{z}^{j} \frac{d \tilde{z}}{F_{z}}=-z^{i} \tilde{z}^{j} \frac{d z}{F_{\tilde{z}}}
$$

со степенями $i=i^{\prime}-1$ и $j=j^{\prime}-1$, где $\left(i^{\prime}, j^{\prime}\right) \in$ N.P. обозначают координаты точек строго внутри многоугольника Ньютона, исключая точки на границе (см. рис. 7$)^{12}$. Подсчитав число целых точек внутри многоугольника, находим, что число линейно независимых голоморфных дифференциалов, или род кривой (5.9),

$$
g=n \tilde{n}-1 .
$$

Для $\tilde{n}=1$ гауссово интегрирование по матрице $\Phi^{\dagger}$ возвращает нас к одноматричной модели с гиперэллиптической кривой рода $n-1$, рассмотренной в разделе 3 , для произвольных $n$ и $\tilde{n}$ кривые (5.9), (5.10), конечно, не являются гиперэллиптическими.

Производящий дифференциал, измеряющий постоянную плотность собственных значений (5.2), имеет вид

$$
d S=\frac{1}{2 \pi i} \tilde{z} d z
$$

12) Например, при $n=2$ существуют три точки внутри многоугольника: $i^{\prime}, j^{\prime}>0$ и $i^{\prime}+j^{\prime} \leqslant 2$, при этом голоморфные дифференциалы нумеруются индексами $i, j \geqslant 0$ и $i+j \leqslant 1$. 


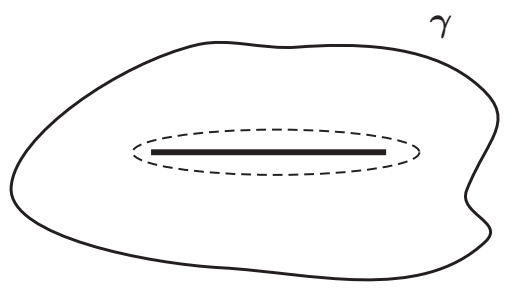

Рис. 8. Граница капли $\gamma$ и разрез многозначной функции $\tilde{z}(z)$ внутри капли. На границе $\gamma$ выполняется равенство $\bar{z}=\tilde{z}(z)$, но это, конечно, не верно на разрезе.

Происхождение этой формулы продемонстрировано на рис. 8, с помощью которого легко убедиться в следующих элементарных соотношениях между двумерными и контурными интегралами:

$$
\int_{\text {drop }} d z \wedge d \bar{z}=\oint_{\boldsymbol{\gamma}} \bar{z} d z=\oint_{\boldsymbol{\gamma}} \tilde{z} d z=\oint_{\text {cut }} \tilde{z} d z,
$$

означающих, что числа заполнения собственных значений могут быть заданы как периоды:

$$
S_{i}=\frac{1}{2 \pi i} \int_{i \text {-th drop }} d z \wedge d \bar{z}=\frac{1}{2 \pi i} \oint_{A_{i}} \tilde{z} d z .
$$

Соотношения (5.14) позволяют оснастить комплексную кривую (5.10) (или кривую (5.9) в асимметричном случае) мероморфным производящим дифференциалом (5.13) и тем самым переформулировать планарную двухматричную модель в геометрических терминах, которые мы обсудили выше. Эти равенства также проясняют соотношение между комплексной и вещественной задачами; сначала мы подробно рассмотрим первую из них, интенсивно используя уравнение алгебраической кривой (5.10), а обсуждение вещественной задачи отложим до раздела 6 , где мы сформулируем ее, следуя [31], в терминах так называемого дубля Шоттки (грубо говоря, двух копий рис. 6) ${ }^{13)}$.

Производные производящего дифференциала (5.13) по коэффициентам уравнения (5.9) могут быть вычислены стандартным способом. Выбирая $z$ в качестве ковариантно постоянной функции при вычислении производных по модулям кривой, для уравнений (5.9), (5.10) можно написать

$$
F_{\tilde{z}} \delta \tilde{z}+\delta F=0
$$

13) В качестве демонстрации нетривиального соотношения между комплексной и вещественной задачами мы представили на рис. 9 вещественное сечение кривой, описываемой конкретным кубическим уравнением. Ясно, что формула (5.12) для комплексной задачи дает $g=3$, т.е. четыре независимых переменных (5.15), отвечающих всем возможным экстремумам. Более того, даже вещественное сечение комплексной кривой в этом примере состоит из четырех несвязанных вещественных контуров. Однако, поскольку у кубического потенциала имеется единственный минимум, только один из этих контуров может находиться на физическом листе для вещественной задачи. Тем не менее все экстремумы неразличимы с точки зрения комплексной задачи и голоморфных данных (5.10), (5.13). "Нефизические" капли могут давать также существенные вклады в непертурбативные явления, но подробное обсуждение этих явлений выходит за рамки данной работы. 
где $\delta F \equiv \sum_{i, j} \delta f_{i j} z^{i} \bar{z}^{j}$ представляет собой вариацию коэффициентов в уравнениях (5.9), (5.10). Для производящего дифференциала (5.13) при этом имеем

$$
\delta(\tilde{z} d z)=-\delta F \frac{d z}{F_{\tilde{z}}}=-\sum_{i, j} \delta f_{i j} \tilde{z}^{j} \frac{z^{i} d z}{F_{\tilde{z}}} .
$$

Выражение (5.17) представляет собой разложение вариации мероморфного дифференциала (5.13) по базису абелевых дифференциалов на кривых (5.9), (5.10). Легко проверить, что коэффициенты $f_{i j}$, отвечающие мероморфным абелевым дифференциалам второго рода, могут быть выражены через параметры потенциала (2.4) двухматричной модели, т.е. через коэффициенты $t$ и $\bar{t}$ его гармонической части [29]. Это немедленно следует из подстановки в уравнения (5.9), (5.10) асимптотического разложения следующей ветви функции $\tilde{z}$ :

$$
\tilde{z}=W^{\prime}(z)+O\left(z^{-1}\right)=\sum_{k=1}^{n+1} k t_{k} z^{k-1}+O\left(z^{-1}\right),
$$

которое приводит к формулам (5.8) и им подобным. Используя (5.18) и (5.13), можно немедленно получить

$$
t_{k}=\left.\frac{1}{2 \pi i k} \operatorname{res}\right|_{z=\infty}\left(z^{-k} \tilde{z} d z\right) .
$$

Остальная часть разложения (5.17) содержит коэффициенты, отвечающие линейным комбинациям абелева дифференциала третьего рода и голоморфных дифференциалов (5.11).

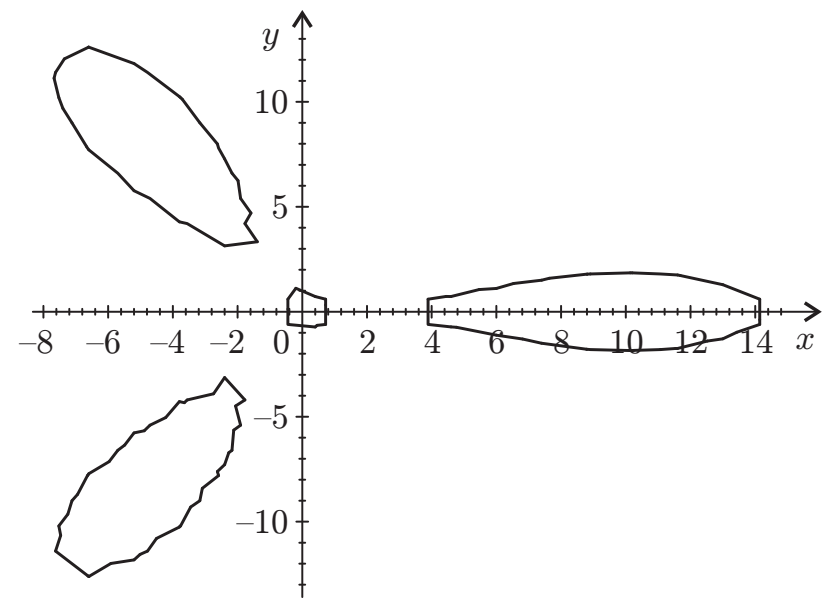

Рис. 9. Решение уравнения $0.11 z^{2} \bar{z}^{2}-z^{3}-\bar{z}^{3}+6 z \bar{z}-6.5=0$, полученное с помощью программы MAPLE.

Зависимость свободной энергии двухматричной модели от чисел заполнения (5.15) и параметров потенциала (2.4), как и в одноматричном случае, может быть сформулирована геометрически:

$$
\frac{\partial \mathcal{F}}{\partial S_{i}}=\frac{1}{2 \pi i} \oint_{B_{i}} \tilde{z} d z
$$


где $\left\{B_{i}\right\}$ являются каноническими дуальными циклами $A_{i} \circ B_{j}=\delta_{i j}$ (см. рис. 3 ) на кривых (5.9), (5.10), а также

$$
\frac{\partial \mathcal{F}}{\partial t_{k}}=\left.\frac{1}{2 \pi i} \operatorname{res}\right|_{z=\infty}\left(z^{k} \tilde{z} d z\right)
$$

Интегрируемость соотношений (5.20) следует из симметрии матрицы периодов кривой (5.10), а интегрируемость уравнений (5.21) - из билинейных соотношений Римана, аналогичных формуле (4.7).

5.3. Структура комплексной кривой двухматричной модели. Чтобы лучше понять структуру кривых (5.9), (5.10), рассмотрим сначала пример с кубическим потенциалом. Выписывая уравнение (5.10) для $n=\tilde{n}=3$ с некоторыми произвольными коэффициентами:

$$
F(z, \tilde{z})=z^{2} \tilde{z}^{2}+a z^{3}+\bar{a} \tilde{z}^{3}+b z^{2} \tilde{z}+\bar{b} z \tilde{z}^{2}+c z^{2}+\bar{c} \tilde{z}^{2}+f z \tilde{z}+q z+\bar{q} \tilde{z}+h=0,
$$

следует согласовать его с асимптотикой (5.18)

$$
\tilde{z}=W^{\prime}(z)-G(z)=\sum_{k=1}^{3} k t_{k} z^{k-1}+O\left(z^{-1}\right) .
$$

Подставляя (5.23) в уравнение (5.22) и собирая коэффициенты при членах $z^{6}, z^{5}$ и $z^{4}$, получим

$$
a=-\frac{1}{3 t_{3}}, \quad b=\frac{2 \tilde{t}_{2}}{3 \tilde{t}_{3}}, \quad c=\frac{\tilde{t}_{1}}{3 \tilde{t}_{3}}-\frac{2 t_{2}}{9 t_{3} \tilde{t}_{3}}+\frac{8 \tilde{t}_{2}^{2}}{9 \tilde{t}_{3}^{2}},
$$

а также комплексно-сопряженные им соотношения, т.е. коэффициенты при старших степенях уравнения (5.22) действительно полностью фиксируются параметрами потенциала (2.4). Четыре младших коэффициента $f, q, \bar{q}$ и $h$ отвечают бипольному дифференциалу (4.6) и трем голоморфным дифференциалам, и их значения зависят от чисел заполнения (5.14), т.е. определяются периодами (5.15) дифференциала $(5.13)^{14)}$. Классические значения этих коэффициентов (при нулевых числах заполнения) могут быть получены из уравнения (5.7).

Посмотрим теперь на кривую (5.22) как на риманову поверхность многозначной функции $\tilde{z}(z)$, которая троекратно накрывает комплексную $z$-плоскость. На первом, физическом листе нет ветвления при $z \rightarrow \infty$, как следует из асимптотики (5.18), (5.23). Однако "комплексно-сопряженная" асимптотика

$$
z=\bar{W}^{\prime}(\tilde{z})+O\left(\tilde{z}^{-1}\right)
$$

на двух нефизических листах $\tilde{z} \propto \sqrt{z}$ утверждает, что их две бесконечности склеены и являются граничной точкой некоторого разреза (см. рис. 10).

14) Заметим, что уравнения кривой (5.10), (5.22) часто пишутся в предположении некоторого условия вещественности на их коэффициенты, но, как обычно, даже в этом случае вариации этих коэффициентов следует рассматривать как независимые комплексные переменные. 


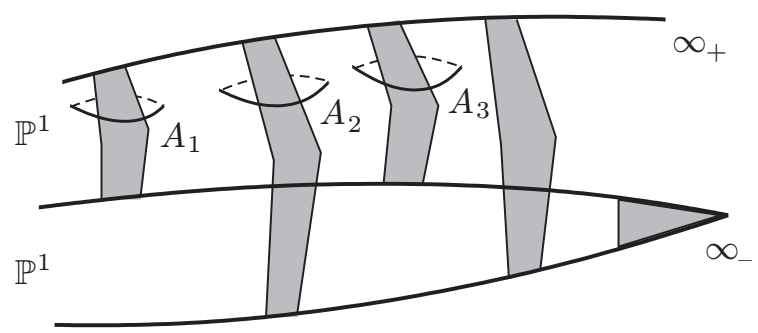

Рис. 10. Кубическая кривая как накрывающая $z$-плоскость.

Точки ветвления в $z$-плоскости определяются нулями дифференциала $d z$, или уравнением $F_{\tilde{z}}=0$. Рассматривая простейший невырожденный случай кривой $(5.22)$

$$
z^{2} \tilde{z}^{2}+a z^{3}+\bar{a} \tilde{z}^{3}+h=0
$$

легко убедиться, что всего существует девять точек ветвления в $z$-плоскости, не считая бесконечной точки $z=\infty$ (естественно, к тому же выводу приводит изучение формулы Кардано или теорем об индексе, см. ниже).

Структура кривой изображена на рис. 10. Кривая представляет собой две копии $\mathbb{P}^{1}$, склеенные по четырем разрезам, т.е. в случае общего положения ее род (5.12) равен $g=3$. На ней две бесконечные точки $z=\infty, \tilde{z}=\infty$, причем одна из них является точкой ветвления. На рис. 10 схематично изображены возможные разрезы и соответствующий им выбор А-циклов. В классическом случае (5.7) вырожденная кривая представляет собой две параболы, пересекающиеся по четырем точкам, и при "квантовом разрешении" сингулярности эти четыре точки превращаются в четыре разреза, соединяющих две сферы $\mathbb{P}^{1}$, как на рис. 10.

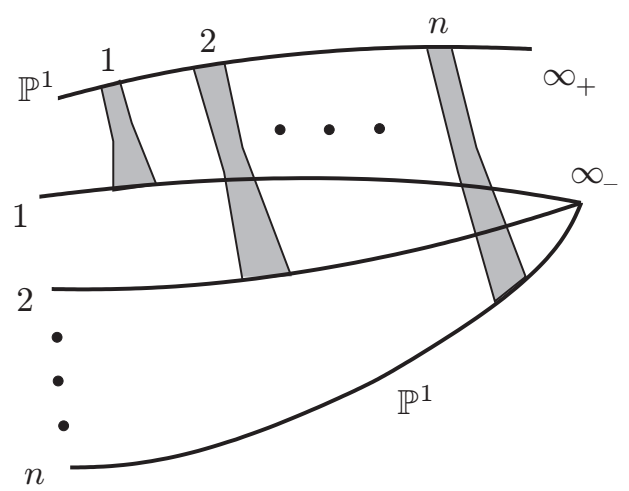

Рис. 11. Общая кривая двухматричной модели с симметричным потенциалом как накрывающая $z$-плоскости. В отличие от рис. 10, здесь каждая толстая линия представляет собой набор из $n$ разрезов.

После этого должно быть совершенно ясно, как кривые (5.9) и (5.10) выглядят для общих полиномиальных потенциалов. Скажем, кривая (5.10) степени $n$, т.е. когда 
$W^{\prime}(z) \sim z^{n}+\cdots$ (см. рис. 11$)$, может быть опять представлена как две копии $\mathbb{P}^{1}$, склеенные $n$ пачками разрезов. Одна из этих сфер $\mathbb{P}^{1}$ отвечает “физическому" $z$ листу, а другая в точке $\infty_{-}$склеена из $n$ копий "нефизических" $z$-листов. Каждая пачка состоит из $n$ разрезов, поэтому полное их число есть $n^{2}$, среди которых можно выбрать $n^{2}-1$ независимых в смысле охватывающих их циклов, т.е. в количестве, равном роду (5.12) этой римановой поверхности.

Дифференциал $d z$ всегда имеет полюс второго порядка в точке $\infty_{+}$на верхнем, или "физическом" листе и полюс порядка $n+1$ в точке $\infty_{-}$, поскольку $\left.z\right|_{\infty_{-}} \propto$ $z^{n}+\cdots$. Вместе это дает $n+3$ полюса, и из теоремы Римана-Роха заключаем, что число точек ветвления или нулей $d z$

$$
\#(d z=0)=n+3+2\left(n^{2}-1\right)-2=2 n^{2}+n-1
$$

что равно девяти при $n=2$. В общей ситуации эта формула дает в точности $2 n^{2}$ точек ветвления, являющихся концами $n^{2}$ простых разрезов, а также $n-1$ точек ветвления, соединенных разрезами с точкой $\infty_{-}$.

5.4. Вырождения кривой максимального рода. До сих пор мы рассматривали комплексную кривую двухматричной модели в положении ее максимально возможного рода (5.12), отвечающего ситуации, когда все экстремумы заполнены собственными значениями с ненулевыми числами заполнения (5.14). Однако во многих случаях, как, например, для вещественной задачи, которая будет обсуждаться ниже, часть экстремумов, отвечающих нестабильным конфигурациям, могут оставаться незаполненными, что для кривых (5.9), (5.10) отвечает их вырождению. Перед тем как перейти к подробному обсуждению вещественной задачи в терминах дубля Шоттки, изучим, следуя [29], как в принципе может вырождаться кривая (5.10).

Род $g=n^{2}-1$ кривой (5.10) уменьшается, если существует нетривиальное решение следующей системы уравнений:

$$
F(z, \tilde{z})=0, \quad d F=\partial_{z} F d z+\partial_{\tilde{z}} F d \tilde{z}=0 .
$$

Эта система налагает связи на коэффициенты $f_{i j}$ уравнения (5.10), которые могут быть найдены путем вычисления результанта уравнений (5.28), или дискриминанта кривой. Однако эти уравнения нельзя эффективно разрешить в случае общего положения.

Чтобы понять, как может вырождаться кривая (5.10), рассмотрим сначала кубический случай (5.22) и допустим, что все коэффициенты этого уравнения вещественны. Тогда легко видеть, что уравнение (5.22) может быть записано в виде

$$
Y^{2}+a X^{3}+c X^{2}+q X+h-\frac{1}{4}((3 a-b) X+2 c-f)^{2} \equiv Y^{2}+P(X)=0,
$$

где $\mathrm{e}^{15)}$

$$
X=z+\tilde{z}, \quad Y=z \tilde{z}-\frac{1}{2}((3 a-b) X+2 c-f) .
$$

15) Для простоты можно также выбрать коэффициенты потенциала (5.24) так, чтобы $3 a=b$ и $2 c=f$. 
Формулы (5.30) демонстрируют, что кривая (5.10) может быть представлена как двойное накрытие тора (5.29) с четырьмя точками ветвления (в которых замена (5.30) становится сингулярной), являющимися решениями уравнения (5.22) после подстановки $\tilde{z}=z$. Таким образом, кривая (5.22) может быть также представлена

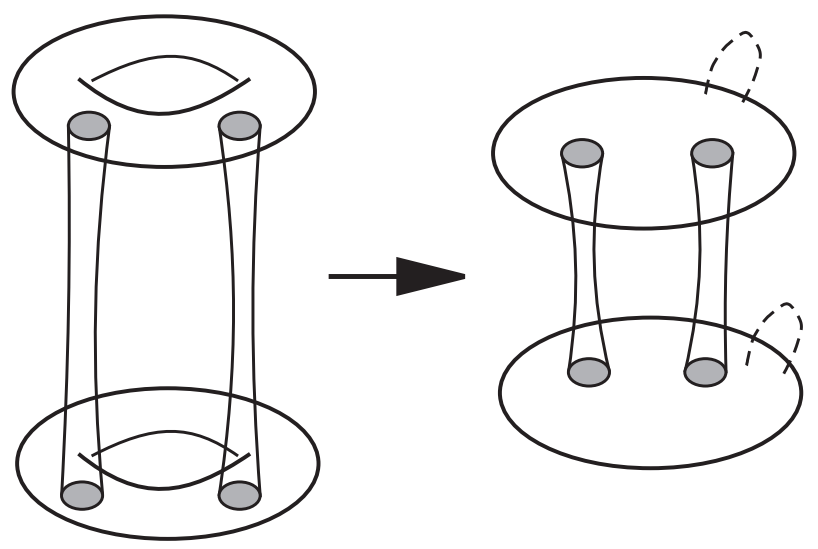

Рис. 12. Кривая (5.22) как двойное накрытие тора. Когда тор (5.29) вырождается, род $g=n^{2}-1=3$ кривой (5.22) падает до $g_{\mathrm{red}}=n-1=1$.

(в дополнение к рис. 10) как два тора, склеенных по двум разрезам (см. рис. 12).

Теперь становится ясно, как эта картина может вырождаться. Переписывая уравнения (5.28) в виде

$$
F_{\tilde{z}}=z F_{Y}+P^{\prime}(X), \quad F_{\tilde{z}}=\tilde{z} F_{Y}+P^{\prime}(X),
$$

немедленно находим, что они приводят либо к $z=\tilde{z}$, либо к уравнению $F_{Y}=0$ или, следовательно, к $P^{\prime}(X)=0$. Во втором случае тор (5.29) вырождается, в то время как условие $z=\tilde{z}$ приводит к вырождению накрытия тора. Если тор вырождается в рациональную кривую, мы получаем вырождение римановой поверхности (5.22), представленное как двойное накрытие сферы с двумя разрезами, т.е. как эллиптическую кривую рода $g=1$ с двумя дополнительными парами сингулярных точек (см. рис. 12).

Наконец, в общем случае кривой (5.10) с вещественными коэффициентами подстановка, аналогичная (5.30), приводит ее к виду

$$
Y^{n}+X^{n+1}+\cdots=0
$$

где многоточием обозначены мономы младших степеней по $X$ и $Y$, а также отсутствуют смешанные члены ${ }^{16)}$. Род кривой (5.32) может быть легко вычислен с помощью многоугольника Ньютона (см. рис. 13), имеем

$$
g_{*}=\frac{n(n-1)}{2} .
$$

\footnotetext{
16) Кривые такого типа рассмотрены в работе [32] в контексте матрично-модельных решений минимальной теории струн, или критических $(p, q)$-точек двумерной гравитации при $|p-q|=1$.
} 


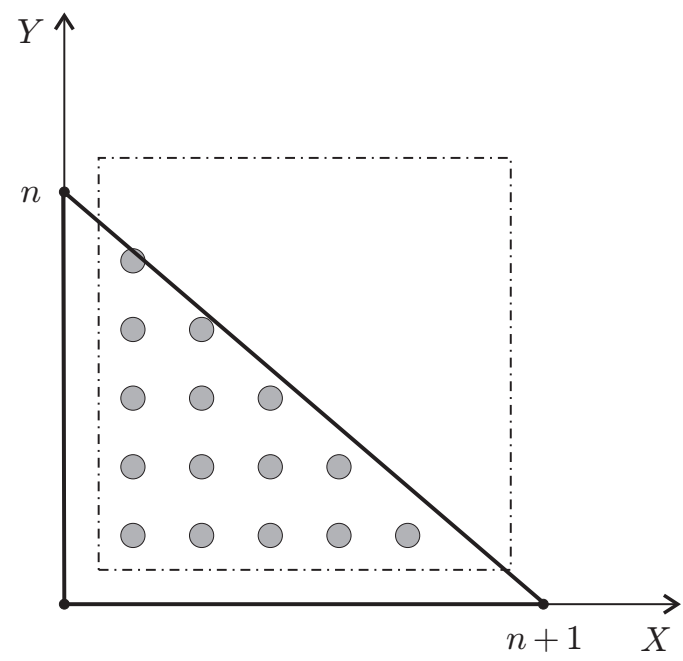

Рис. 13. Многоугольник Ньютона для кривой (5.32) приводит к роду $g_{*}=n(n-1) / 2$.

Тем же способом можно представить общую кривую двухматричной модели (5.10) как двойное накрытие римановой поверхности (5.32) с $2 n$ точками ветвления. Действительно, формула Римана-Гурвица

$$
2-2 g=\# S \cdot\left(2-2 g_{0}\right)-\# \text { B.P. }
$$

где \# $S$ означает число листов накрытия, а \# В.Р. - число точек ветвления, дает \# В.Р. $=2 n$ для $g=n^{2}-1$ и $g_{0}=g_{*}$. Это означает, что общая кривая двухматричной модели (5.10) может быть представлена как двойное накрытие кривой (5.32) с $n$ разрезами, а когда кривая (5.32) вырождается в рациональную, род кривой (5.10) падает до $g_{\mathrm{red}}=n-1$, т.е. растет линейным образом с ростом старшей степени потенциала, как в случае одноматричной модели (см. рис. 14). В заключение данного раздела добавим несколько слов о рациональных вырождениях модели (5.10), т.е. когда ее род $g=0$. Частным случаем такого полного вырождения является полностью вырожденная классическая кривая (5.7), и рациональный случай легко выписывается для общих значений коэффициентов уравнения (5.10), т.е. без всяких условий вещественности.

В этой ситуации уравнение кривой (5.10) может быть разрешено с помощью обобщенного рационального конформного отображения

$$
z=r w+\sum_{k=0}^{n} \frac{u_{k}}{w^{k}}, \quad \tilde{z}=\frac{r}{w}+\sum_{k=0}^{n} \bar{u}_{k} w^{k}
$$

с униформизующим параметром $w$, а подстановка формул (5.35) в уравнение (5.10) приводит к системе уравнений, выражающей все коэффициенты $f_{i j}$ в терминах параметров конформного отображения (5.35) (их явный вид можно найти в приложении 1). 


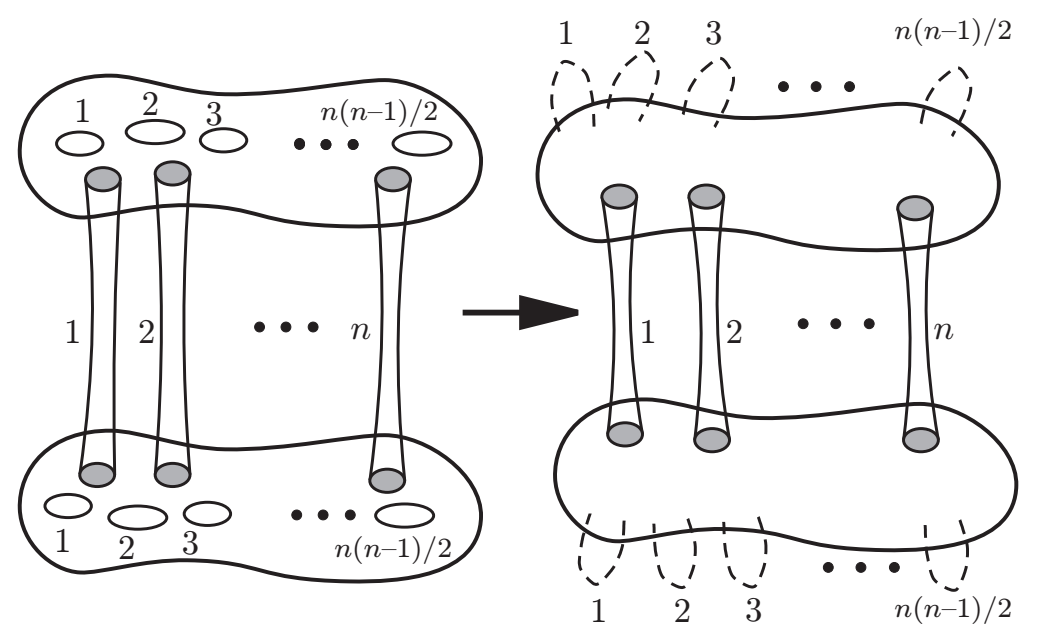

Рис. 14. Общая кривая (5.10) как двойное накрытие кривой (5.32) рода $g_{*}=n(n-1) / 2$.

\section{6. ДВУХМАТРИЧНАЯ МОДЕЛЬ И ЗАДАЧА ДИРИХЛЕ}

В данном разделе мы продолжим изучение статсуммы матричной модели (2.5). Оказывается, что для вещественной задачи, когда максимальный род (5.12) гладкой кривой не может быть достигнут при заполнении лишь минимумов потенциала (2.4), наиболее эффективный способ отождествить свободную энергию с квазиклассической тау-функцией заключается в использовании ее связи с задачей Дирихле для границы области распределения собственных значений, показанной на рис. 6. Для связных (и односвязных) областей эта задача решается в терминах конформных отображений (5.35), но в случае многих капель или многосвязных областей решение задачи Дирихле существенно использует технику, введенную в разделе 4.

6.1. Задача Дирихле и интегрируемость. Сначала мы сформулируем некоторые результаты работ [31], [33] в форме, наиболее тесно связанной с квазиклассическими решениями матричных моделей. Как было упомянуто выше, распределение собственных значений для вещественной задачи в двухматричной модели (2.5) принимает форму некоторых капель в комплексной плоскости (см. рис. 6) с постоянной плотностью (5.2) для потенциала (2.4). Плотность остается постоянной, а квазиклассическая иерархия описывает теперь деформацию формы этих капель, информация о которой может содержаться в решении соответствующей граничной задачи Дирихле.

Начнем с решения с единственной каплей, отвечающей связанной области $\mathrm{D}$, ограниченной простой гладкой кривой. Следуя [31], [33], мы рассмотрим внешнюю задачу Дирихле в области $\mathrm{D}^{\mathrm{c}}=\mathbb{C} \backslash \mathrm{D}$

$$
u(z)=-\frac{1}{2 \pi} \oint_{\partial \mathrm{D}} u_{0}(\xi) \partial_{n} G(z, \xi)|d \xi|,
$$




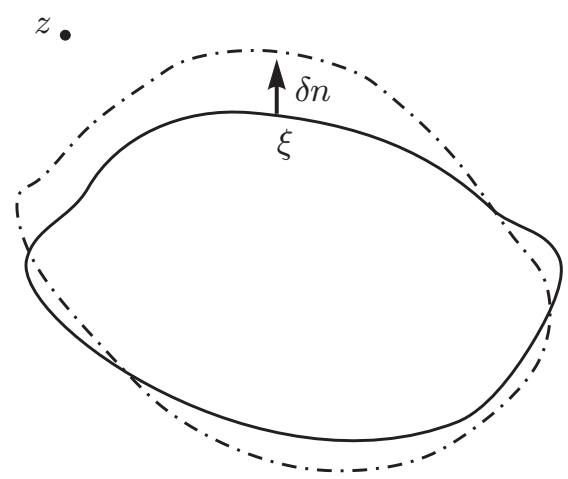

Рис. 15. Деформация области с нормальным смещением $\delta n(\xi)$.

где $G\left(z, z^{\prime}\right)$ представляет собой функцию Грина задачи Дирихле с граничными условиями $\left.G\left(z, z^{\prime}\right)\right|_{z \in \partial \mathrm{D}}=\left.G\left(z, z^{\prime}\right)\right|_{z^{\prime} \in \partial \mathrm{D}}=0$, и предположим, что точка $z=0$ находится внутри области D. Тогда область $\mathrm{D}^{\mathrm{c}}$ содержит точку $z=\infty$. Воспользуемся вариационной формулой Адамара, выражающей вариацию функции Грина задачи Дирихле $G\left(z, z^{\prime}\right)$ относительно малых деформаций области D через саму функцию Грина:

$$
\delta G\left(z, z^{\prime}\right)=\frac{1}{2 \pi} \oint_{\partial \mathrm{D}} \partial_{n} G(z, \xi) \partial_{n} G\left(z^{\prime}, \xi\right) \delta n(\xi)|d \xi| .
$$

Здесь $\delta n(\xi)$ представляет собой нормальное смещение границы при деформации в граничной точке $\xi$ (см. рис. 15$)$, а $\partial_{n}$ обозначает соответствующую нормальную производную.

Решение задачи Дирихле с единственной каплей эквивалентно [33] нахождению конформного отображения из области $\mathrm{D}^{\mathrm{c}}$ в дополнение к единичному кругу $|w|>1$ или любой другой исходной области, для которой функция Грина известна явно. Такое конформное отображение $w(z)$ существует в силу теоремы Римана об отображениях, при этом

$$
G\left(z, z^{\prime}\right)=\ln \left|\frac{w(z)-w\left(z^{\prime}\right)}{w(z) \overline{w\left(z^{\prime}\right)}-1}\right|,
$$

где черта означает комплексное сопряжение. Примерами таких отображений могут служить обратные к рациональным функциям (5.35), и это практически решает задачу для матричной модели (2.5) с полиномиальным потенциалом и распределением в виде единственной капли.

Обозначим через $t_{k}$ моменты области $\mathrm{D}^{\mathrm{c}}=\mathbb{C} \backslash \mathrm{D}$, определенные по отношению к гармоническим функциям $\left\{z^{-k} / k\right\}$ :

$$
t_{k}=-\frac{1}{\pi k} \int_{\mathrm{D}^{\mathrm{c}}} z^{-k} d^{2} z, \quad k=1,2, \ldots,
$$

а через $\bar{t}_{k}-$ комплексно-сопряженные моменты, т.е.

$$
\bar{t}_{k}=-\frac{1}{\pi k} \int_{\mathrm{D}^{\mathrm{c}}} \bar{z}^{-k} d^{2} z ;
$$


совпадение обозначений с гармоническими параметрами потенциала матричной модели (2.4), естественно, не случайно. Формула Стокса преобразует интегралы в (6.4) в контурные интегралы, например

$$
t_{k}=\frac{1}{2 \pi i k} \oint_{\partial \mathrm{D}} z^{-k} \bar{z} d z=\frac{1}{2 \pi i k} \oint z^{-k} \tilde{z} d z,
$$

обеспечивая, в частности, регуляризацию возможных расходимостей в (6.4) и непосредственно связывая их с временами матричной модели (5.19), если рассматривать $\tilde{z}(z)$ как аналитическое продолжение функции $\bar{z}(z)$ с границы $\partial \mathrm{D}$ (см. рис. 8). Кроме того, обозначим через $t_{0}$ площадь области $\mathrm{D}$ :

$$
t_{0}=\frac{1}{\pi} \int_{\mathrm{D}} d^{2} z
$$

- полное число собственных значений, распределенных в области с постоянной плотностью (5.2).

Гармонические моменты (6.4) области $\mathrm{D}^{\mathrm{c}}$ являются коэффициентами разложения Тейлора потенциала

$$
\Phi(z, \bar{z})=-\frac{2}{\pi} \int_{\mathrm{D}} \ln \left|z-z^{\prime}\right| d^{2} z^{\prime}
$$

индуцированного областью D и напрямую связанного с потенциалом (2.4) двухматричной модели

$$
V(z, \bar{z})=\Phi(0)-\Phi(z, \bar{z})=z \bar{z}-W(z)-\bar{W}(\bar{z})=|z|^{2}-\sum_{k \geqslant 1}\left(t_{k} z^{k}+\bar{t}_{k} \bar{z}^{k}\right) .
$$

Производная потенциала, или резольвента (5.6),

$$
\partial_{z} \Phi(z)=-\frac{1}{\pi} \int_{\mathrm{D}} \frac{d^{2} z^{\prime}}{z-z^{\prime}}=-\frac{1}{\pi} G(z)
$$

непрерывна на границе и голоморфна при $z \in \mathrm{D}^{\mathrm{c}}$, в то время как для $z \in \mathrm{D}$ голоморфна функция $\partial_{z} \Phi+\bar{z}$. Если граница является аналитической вещественной кривой, то обе эти функции продолжаются аналитически, и существует функция $\tilde{z}(z)$, аналитичная по крайней мере в некоторой полосе вокруг границы и равная $\tilde{z}(z)=\bar{z}$ на самой границе. Другими словами, аналитическое продолжение $\tilde{z}(z)$ функции $\bar{z}$ с граничного контура, непосредственно связанное с производящим дифференциалом (5.13), полностью определяет форму границы и называется в данном контексте функцией Шварца.

Основным фактом теории деформации замкнутых гладких кривых является то, что комплексные моменты (6.4), (6.5), дополненные вещественной переменной (6.6), образуют набор "хороших" локальных координат в пространстве модулей таких кривых. Более того, этот набор не переполнен, как следует из явного построения соответствующих векторных полей (подробнее см. работу [31] и цитированную в ней литературу). Доказательство этого утверждения основано на наблюдении, что разница граничных значений $\partial_{t} C^{ \pm}(\zeta) d \zeta$ производной интеграла Коши

$$
C(z) d z=\frac{d z}{2 \pi i} \oint_{\partial \mathrm{D}} \frac{\bar{\zeta} d \zeta}{\zeta-z}
$$


представляет собой чисто мнимый дифференциал на границе области D:

$$
\partial_{t} C(z) d z=\frac{d z}{2 \pi i} \oint_{\partial \mathrm{D}}\left(\frac{\bar{\zeta}_{t} \zeta_{\sigma}+\bar{\zeta} \zeta_{t, \sigma}}{\zeta-z}-\frac{\bar{\zeta} \zeta_{\sigma} \zeta_{t}}{(\zeta-z)^{2}}\right) d \sigma=\frac{d z}{2 \pi i} \oint_{\partial \mathrm{D}}\left(\frac{\bar{\zeta}_{t} \zeta_{\sigma}-\bar{\zeta}_{\sigma} \zeta_{t}}{\zeta-z}\right) d \sigma,
$$

где $\zeta(\sigma ; t)$ - параметризация вещественной кривой $\partial \mathrm{D}(t)$. Поэтому дифференциал

$$
\left(\partial_{t} C^{+}(\zeta)-\partial_{t} C^{-}(\zeta)\right) d \zeta=\partial_{t} \bar{\zeta} d \zeta-\partial_{t} \zeta d \bar{\zeta}=2 i \operatorname{Im}\left(\partial_{t} \bar{\zeta} d \zeta\right)
$$

действительно является чисто мнимым, и если $t$-деформация сохраняет все моменты $t_{k}, k \geqslant 0$, то дифференциал $\partial_{t} \bar{\zeta} d \zeta-\partial_{t} \zeta d \bar{\zeta}$ продолжается до голоморфного в $\mathrm{D}^{\mathrm{c}} . \mathrm{B}$ самом деле, если $|z|<|\zeta|$ для любых $\zeta \in \partial \mathrm{D}$, то можно написать разложение

$$
\partial_{t} C^{+}(z) d z=\frac{\partial}{\partial t}\left(\frac{d z}{2 \pi i} \sum_{k=0}^{\infty} z^{k} \oint_{\partial \mathrm{D}} \zeta^{-k-1} \bar{\zeta} d \zeta\right)=\sum_{k=1}^{\infty} k\left(\partial_{t} t_{k}\right) z^{k-1} d z=0
$$

и поскольку функция $C^{+}$аналитична в $\mathrm{D}$, приходим к заключению, что $\partial_{t} C^{+} \equiv 0$. Выражение $\partial_{t} \bar{\zeta} d \zeta-\partial_{t} \zeta d \bar{\zeta}$ является граничным значением дифференциала $-\partial_{t} C^{-}(z) d z$ с единственно возможным простым полюсом в бесконечности и голоморфным везде, кроме бесконечности, в $\mathrm{D}^{\mathrm{c}}$. При этом из равенства

$$
\partial_{t} t_{0}=\frac{1}{2 \pi i} \oint_{\partial \mathrm{D}}\left(\partial_{t} \bar{\zeta} d \zeta-\partial_{t} \zeta d \bar{\zeta}\right)=0
$$

следует, что вычет в $z=\infty$ равен нулю, а следовательно, дифференциал $\partial_{t} C^{-}(z) d z$ является голоморфным. Далее, любой голоморфный дифференциал, чисто мнимый вдоль границы односвязной области, обязательно равен нулю согласно принципу симметрии Шварца на дубле Шоттки, получающемся приклеиванием к области $\mathrm{D}^{\mathrm{c}}$ ее комплексно-сопряженной копии вдоль границы (согласно принципу симметрии Шварца $\partial_{t} C^{-} d z$ продолжается до глобально определенного голоморфного дифференциала на сфере $\mathbb{P}^{1}$, т.е. должен обращаться в нуль по теореме Римана-Роха).

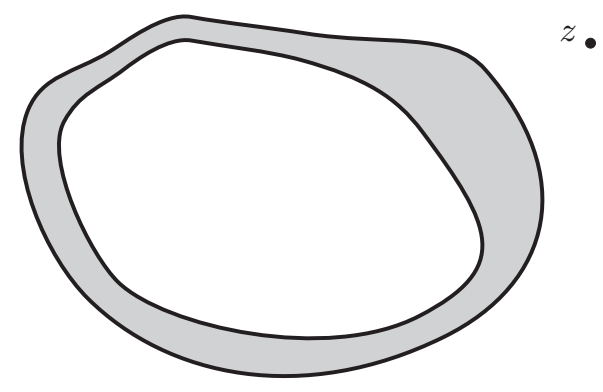

Рис. 16. Элементарная деформация относительно точки $z$.

Для фиксированной точки $z \in \mathrm{D}^{\mathrm{c}}$ можно рассмотреть специальную инфинитезимальную деформацию области, при которой нормальное смещение границы пропорционально градиенту функции Грина $G(z, \xi)$ (см. рис. 16):

$$
\delta_{z} n(\xi)=-\frac{\epsilon}{2} \partial_{n} G(z, \xi)
$$


которую будем называть, следуя работе [31], элементарной деформацией относительно точки $z$. Легко видеть, что формулы

$$
\begin{aligned}
& \delta_{z} t_{0}=\frac{1}{\pi} \oint_{\partial \mathrm{D}} \delta n(\xi)|d \xi|=-\frac{\epsilon}{2 \pi} \oint \partial_{n} G(z, \xi)|d \xi|=\epsilon \\
& \delta_{z} t_{k}=\frac{1}{\pi k} \oint_{\partial \mathrm{D}} \xi^{-k} \delta n(\xi)|d \xi|=-\frac{\epsilon}{2 \pi k} \oint \xi^{-k} \partial_{n} G(z, \xi)|d \xi|=\frac{\epsilon}{k} z^{-k}
\end{aligned}
$$

являются прямым следствием формулы Дирихле $(6.1)^{17)}$. Рассмотрим теперь изменение $\delta_{z} X$ любого функционала $X=X(\mathbf{t})$ при элементарной деформации относительно точки $z$ в главном порядке по $\epsilon$, т.е.

$$
\delta_{z} X=\sum_{k} \frac{\partial X}{\partial t_{k}} \delta_{z} t_{k}=\epsilon \nabla(z) X,
$$

где дифференциальный оператор $\nabla(z)$ определен следующим образом:

$$
\nabla(z)=\partial_{t_{0}}+\sum_{k \geqslant 1}\left(\frac{z^{-k}}{k} \partial_{t_{k}}+\frac{\bar{z}^{-k}}{k} \partial_{\bar{t}_{k}}\right) .
$$

Заметим, что элементарную деформацию можно интуитивно понимать [33] как "шишку" на границе области, гармонически продолженную в $\mathrm{D}^{c}$ (см. рис. 17). Действительно, для этих двух деформаций имеем

$$
\delta_{z} \propto \oint \partial_{n} G(z, \xi) \delta^{\text {bump }}(\xi)|d \xi|,
$$

и деформацию типа "шишки" следует понимать как предел $\delta_{z}$ при стремлении точки $z$ к границе области $\partial \mathrm{D}$.

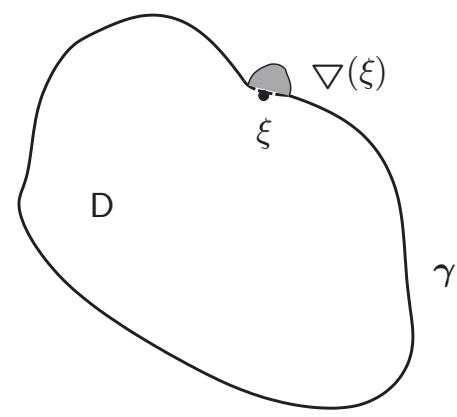

Рис. 17. Деформация в граничной точке $\xi$.

Зафиксируем теперь три точки $z_{1}, z_{2}, z_{3} \in \mathbb{C} \backslash \mathrm{D}$ и вычислим $\delta_{z_{i}} G\left(z_{j}, z_{k}\right)$ с помощью формулы Адамара (6.2). Используя (6.17), можно отождествить результат с

17)Заметим, что элементарная деформация относительно точки $\infty$ сохраняет все моменты, кроме $t_{0}$. Поэтому деформация, которая меняет только $t_{0}$, отвечает нормальному смещению $\delta n(\xi)=-(\epsilon / 2) \partial_{n} G(\infty, \xi)$. 
действием векторного поля $\nabla\left(z_{i}\right)$ на функцию Грина:

$$
\nabla\left(z_{3}\right) G\left(z_{1}, z_{2}\right)=-\frac{1}{4 \pi} \oint_{\partial \mathrm{D}} \partial_{n} G\left(z_{1}, \xi\right) \partial_{n} G\left(z_{2}, \xi\right) \partial_{n} G\left(z_{3}, \xi\right)|d \xi|
$$

Замечательно, что правая часть формулы (6.19) симметрична по всем трем аргументам, т.е.

$$
\nabla\left(z_{1}\right) G\left(z_{2}, z_{3}\right)=\nabla\left(z_{2}\right) G\left(z_{3}, z_{1}\right)=\nabla\left(z_{3}\right) G\left(z_{1}, z_{2}\right) .
$$

Это соотношение позволяет представить деформацию задачи Дирихле (6.1) в терминах интегрируемой иерархии нелинейных дифференциальных уравнений [33], и при этом формула (6.20) является условием интегрируемости этой иерархии. Из соотношения (6.20) следует, что существует функция $\mathcal{F}_{\mathrm{D}}=\mathcal{F}_{\mathrm{D}}(\mathbf{t})$ моментов (6.4), (6.6) такая, что ${ }^{18)}$

$$
G\left(z, z^{\prime}\right)=\ln \left|\frac{1}{z}-\frac{1}{z^{\prime}}\right|+\frac{1}{2} \nabla(z) \nabla\left(z^{\prime}\right) \mathcal{F}_{\mathrm{D}},
$$

т.е. функция Грина является второй $\nabla(z)$-производной, с точностью до члена, не зависящего от времен, которая определяется из граничных условий в совпадающих точках $z=z^{\prime}$ и при $z, z^{\prime} \rightarrow \infty$.

Формула (6.21) позволяет получить представление функции $\mathcal{F}_{\mathrm{D}}=\mathcal{F}_{\mathrm{D}}(\mathbf{t})$ в виде двойного интеграла по области D. Положим $\tilde{\Phi}(z)=\nabla(z) \mathcal{F}_{\mathrm{D}}$, эта функция определяется по ее вариации относительно элементарной деформации

$$
\delta_{\zeta} \tilde{\Phi}(z)=-2 \epsilon \ln \left|\zeta^{-1}-z^{-1}\right|+2 \epsilon G(\zeta, z),
$$

которая определяется из уравнения (6.21) с помощью (6.17). Это позволяет отождествить $\tilde{\Phi}(z)$ с модифицированным потенциалом области $\tilde{\Phi}(z)=\Phi(z)-\Phi(0)+t_{0} \ln |z|^{2}$, где определение $\Phi$ дано в (6.7). Поэтому можно написать

$$
\nabla(z) \mathcal{F}_{\mathrm{D}}=\tilde{\Phi}(z)=-\frac{2}{\pi} \int_{\mathrm{D}} \ln \left|z^{-1}-\zeta^{-1}\right| d^{2} \zeta=v_{0}+2 \operatorname{Re} \sum_{k>0} \frac{v_{k}}{k} z^{-k} .
$$

Последнее равенство следует воспринимать как разложение Тейлора вокруг бесконечности, а коэффициенты $v_{k}$ являются моментами области D (двойственными гармоническими моментами к (6.4)), определенными посредством формул

$$
v_{k}=\frac{1}{\pi} \int_{\mathrm{D}} z^{k} d^{2} z=\partial_{t_{k}} \mathcal{F}_{\mathrm{D}}, \quad k>0, \quad v_{0}=-\Phi(0)=\frac{2}{\pi} \int_{\mathrm{D}} \ln |z| d^{2} z=\partial_{t_{0}} \mathcal{F}_{\mathrm{D}}
$$

т.е. моменты дополнительной области $\mathrm{D}$ полностью определяются функцией $\mathcal{F}_{\mathrm{D}}$ гармонических моментов области $\mathrm{D}^{\mathrm{c}}$. Переписанные в виде контурных интегралов формулы (6.24) позволяют идентифицировать функцию $\mathcal{F}_{\mathrm{D}} \equiv \mathcal{F}$ с квазиклассической тау-функцией, отвечающей планарному решению матричной модели (2.5) с единственной каплей.

Аналогичным образом получается и интегральное представление самой тау-функции. Используя то, что элементарная деформация $\delta_{\xi}$ или действие оператора $\nabla(\xi)$,

\footnotetext{
18)Эта формула была впервые замечена Тахтаджяном, см. обсуждение в работе [34].
} 
примененного в граничной точке $\xi \in \partial \mathrm{D}$ (где $G(z, \xi)=0$ ), является деформацией типа "шишки" (см. рис. 17), и интерпретируя (6.23) как вариацию $\delta_{z} \mathcal{F}_{\mathrm{D}}$, получаем следующее двойное интегральное представление для тау-функции:

$$
\mathcal{F}_{\mathrm{D}}=\frac{1}{2 \pi} \int_{\mathrm{D}} \tilde{\Phi}(z) d^{2} z=-\frac{1}{\pi^{2}} \int_{\mathrm{D}} \int_{\mathrm{D}} \ln \left|z^{-1}-\zeta^{-1}\right| d^{2} z d^{2} \zeta
$$

Это выражение представляет собой не что иное, как непрерывный аналог эффективного потенциала (5.4), вычисленного на его экстремали, и эта формула останется без изменений в случае несвязного носителя (или многосвязной области), хотя отождествление этой функции со свободной энергией матричной модели (2.5) потребует аккуратности.

6.2. Многосвязный случай и дубль Шоттки. Перейдем теперь к общему решению с несвязным носителем. Пусть $\mathrm{D}_{\alpha}, \alpha=0,1, \ldots, g$, представляет собой набор из $g+1$ непересекающихся ограниченных областей в комплексной плоскости с гладкими границами $\partial \mathrm{D}_{\alpha}$ такими, что $\mathrm{D}=\bigcup_{\alpha=0}^{g} \mathrm{D}_{\alpha}$, а их дополнение $\mathrm{D}^{\mathrm{c}}=\mathbb{C} \backslash \mathrm{D}$ становится многосвязной областью в комплексной плоскости (см. рис. 18). Обозначим через $B_{\alpha}$ класс гомологий граничных кривых $\partial \mathrm{D}_{\alpha}$, предполагая, что они ориентированы положительно как границы $\mathrm{D}^{\mathrm{c}}$, так что $\bigcup_{\alpha=0}^{g} B_{\alpha} \simeq-\partial \mathrm{D}$, или что каждый $B_{\alpha}$-цикл ориентирован по часовой стрелке.
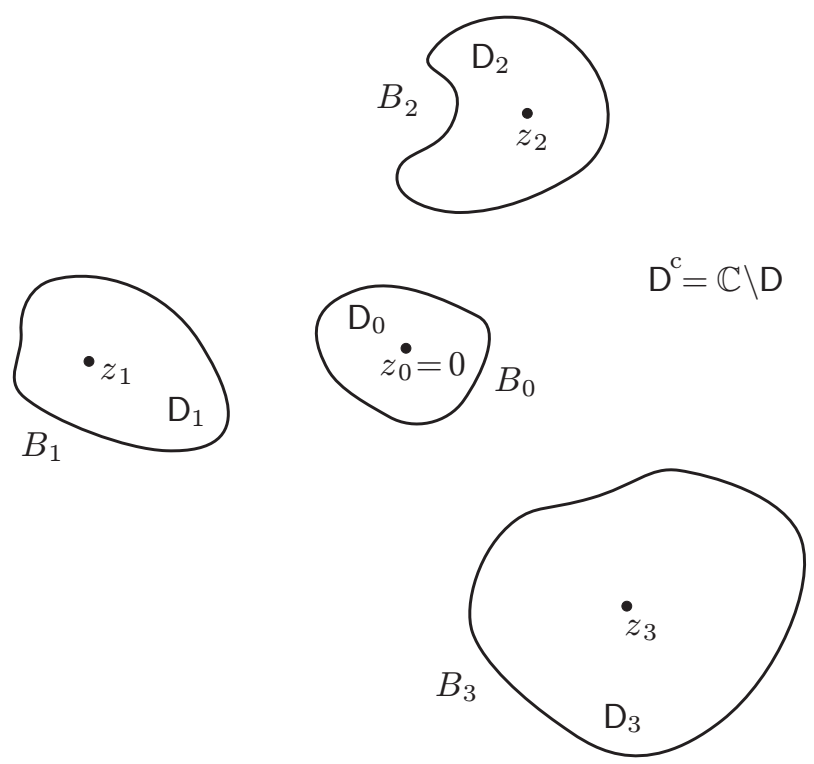

Рис. 18. Многосвязная область $\mathrm{D}^{\mathrm{c}}=\mathbb{C} \backslash \mathrm{D}$ для $g=3$. Область $\mathrm{D}=\bigcup_{\alpha=0}^{3} \mathrm{D}_{\alpha}$ состоит из $g+1=4$ несвязных областей $\mathrm{D}_{\alpha}$ с границами $\partial \mathrm{D}_{\alpha}$. Чтобы определить полный набор гармонических моментов, требуются дополнительные точки $z_{\alpha} \in \mathrm{D}_{\alpha}$, которые всегда расположены внутри соответствующих капель. 
По сравнению с односвязным случаем ничего не меняется в постановке стандартной задачи Дирихле. Определение функции Грина и формула (6.1) для решения задачи Дирихле с помощью функции Грина остаются без изменений. Однако разница заключается в наборе гармонических функций: любая гармоническая функция по-прежнему является вещественной частью аналитической функции, но в многосвязном случае эти аналитические функции не обязательно однозначны (только их вещественные части обязаны быть однозначными). Другими словами, гармонические функции могут иметь ненулевые "периоды" вдоль нетривиальных циклов ${ }^{19)}$, в данном случае вдоль граничных контуров $B_{\alpha}$. В общем случае функция Грина имеет ненулевые периоды по всем граничным контурам, поэтому в многосвязном случае естественно ввести новые объекты, связанные с периодами функции Грина.

Прежде всего, введем гармоническую меру $\varpi_{\alpha}(z)$, отвечающую граничной компоненте $\partial \mathrm{D}_{\alpha}$, которая является гармонической функцией в $\mathrm{D}^{\mathrm{c}}$, равной единице на границе $\partial \mathrm{D}_{\alpha}$ и нулю на остальных граничных контурах. Другими словами, гармоническая мера решает конкретную задачу Дирихле, для которой из формулы (6.1) получаем

$$
\varpi_{\alpha}(z)=\frac{1}{2 \pi} \oint_{\partial \mathrm{D}_{\alpha}} \partial_{n} G(z, \zeta)|d \zeta|, \quad \alpha=1,2, \ldots, g,
$$

т.е. гармоническая мера является периодом функции Грина по одному из аргументов. Из принципа максимума для гармонических функций следует, что $0<$ $\varpi_{\alpha}(z)<1$ во внутренних точках, а кроме того, очевидно, что $\sum_{\alpha=0}^{g} \varpi_{\alpha}(z)=1$. В дальнейшем мы будем пользоваться линейно независимыми функциями $\varpi_{\alpha}(z)$, $\alpha=1,2, \ldots, g$.

Далее, вычислив периоды, определим матрицу

$$
T_{\alpha \beta}=\frac{i}{2} \oint_{\partial \mathrm{D}_{\beta}} \partial_{n} \varpi_{\alpha}(\zeta)|d \zeta|, \quad \alpha, \beta=1,2, \ldots, g,
$$

которая является симметричной, невырожденной и положительно определенной (мнимой) матрицей. В дальнейшем будет ясно, что матрица (6.27) может быть идентифицирована с матрицей периодов (4.2) голоморфных дифференциалов на дубле Шоттки области $\mathrm{D}^{\mathrm{c}}$.

Для гармонической меры и матрицы периодов существуют дополнительные формулы, подобные формуле Адамара (6.2):

$$
\begin{aligned}
\delta \varpi_{\alpha}(z) & =\frac{1}{2 \pi} \oint_{\partial \mathrm{D}} \partial_{n} G(z, \xi) \partial_{n} \varpi_{\alpha}(\xi) \delta n(\xi)|d \xi| \\
\delta T_{\alpha \beta} & =\frac{i}{2} \oint_{\partial \mathrm{D}} \partial_{n} \varpi_{\alpha}(\xi) \partial_{n} \varpi_{\beta}(\xi) \delta n(\xi)|d \xi| .
\end{aligned}
$$

Вывести их можно или непосредственным варьированием формул (6.26) и (6.27) с использованием формулы Адамара, или с помощью рис. 16, 17.

\footnotetext{
19) Под "периодами" гармонической функции $f$ подразумеваются интегралы $\oint \partial_{n} f d l$ по нетривиальным циклам.
} 
С каждой планарной (в том числе и многосвязной) областью можно ассоциировать дубль Шоттки - компактную риманову поверхность $\Sigma$, оснащенную антиголоморфной инволюцией такой, что граница области является множеством неподвижных точек этой инволюции. Дубль Шоттки области $\mathrm{D}^{\mathrm{c}}$ можно представить как две ее копии (верхний и нижний листы дубля), склеенные по границам и с добавленными двумя бесконечностями ( $\infty$ и $\bar{\infty})$. При этом в качестве голоморфной координаты на верхнем листе выбирается $z$, наследованная от $\mathrm{D}^{\mathrm{c}}$, в то время как голоморфной координатой на другом листе является $\bar{z}^{20)}$. Дубль Шоттки многосвязной области $\mathrm{D}^{\mathrm{c}}$ представляет собой риманову поверхность $\Sigma$ рода $g=\#\left\{\mathrm{D}_{\alpha}\right\}-1$. Мероморфной функцией на дубле является пара мероморфных функций $f, \tilde{f}$ на $\mathrm{D}^{c}$ таких, что $f(z)=\tilde{f}(\bar{z})$ на границе $\partial \mathrm{D}^{\mathrm{c}}$. Аналогично мероморфный дифференциал на дубле представляет собой пару мероморфных дифференциалов $f(z) d z$ и $\tilde{f}(\bar{z}) d \bar{z}$ таких, что для них выполняется равенство $f(z) d z=\tilde{f}(\bar{z}) d \bar{z}$ вдоль граничных контуров.
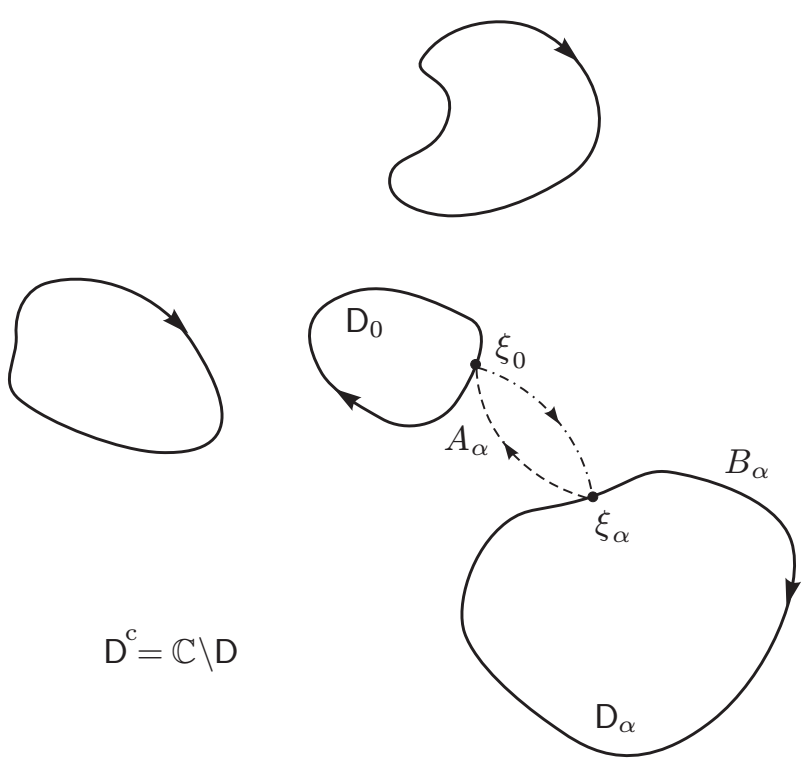

Рис. 19. Область $\mathrm{D}^{\mathrm{c}}$ и $A_{\alpha}$-цикл, который в одну сторону проходит по верхнему листу, а назад - по нижнему листу дубля Шоттки области $\mathrm{D}^{\mathrm{c}}$. При таком выборе получается форма пересечений $A_{\alpha} \circ B_{\beta}=\delta_{\alpha \beta}$ при $\alpha, \beta=1,2, \ldots, g$.

Фиксируем на дубле канонический базис циклов: $B_{\alpha}$-циклы, гомологически эквивалентные границам дырок $-\partial \mathrm{D}_{\alpha}$ при $\alpha=1,2, \ldots, g$, и $A_{\alpha}$-циклы, соединяющие каждую $\alpha$-ю дырку с выделенной “нулевой”. Более точно, при вычислении интегралов следует зафиксировать точки $\xi_{\alpha}$ на границах, тогда $A_{\alpha}$-цикл можно выбрать начинающимся в точке $\xi_{0}$, доходящим до $\xi_{\alpha}$ по верхнему (голоморфному) листу дубля и возвращающимся назад таким же путем по нижнему листу, на котором голоморфной координатой является $\bar{z}$ (см. рис. 19). Гармонические меры $\varpi_{\alpha}$

\footnotetext{
20) Более точно, настоящими координатами являются $1 / z$ и $1 / \bar{z}$, которые имеют нули первого порядка вместо полюсов при $z=\infty$ и $\bar{z}=\bar{\infty}$, соответственно.
} 
можно представить как вещественные части $\varpi_{\alpha}(z)=\omega_{\alpha}(z)+\overline{\omega_{\alpha}(z)}$ голоморфных многозначных функций на $\mathrm{D}^{\mathrm{c}}$. Их дифференциалы $d \omega_{\alpha}$ голоморфны и однозначны на $\mathrm{D}^{\mathrm{c}}$, а также являются чисто мнимыми вдоль граничных контуров, поэтому их можно продолжить как голоморфные дифференциалы $-d \overline{\omega_{\alpha}(z)}$ на нижний лист. В действительности таким образом мы построили канонический базис голоморфных дифференциалов (4.1) на дубле $\Sigma$ для выбранного выше базиса канонических циклов, поскольку

$$
\begin{aligned}
\oint_{A_{\alpha}} d \omega_{\beta} & =\int_{\xi_{0}}^{\xi_{\alpha}} d \omega_{\beta}(z)+\int_{\xi_{\alpha}}^{\xi_{0}}\left(-\overline{\omega_{\beta}(z)}\right)= \\
& =2 \operatorname{Re} \int_{\xi_{0}}^{\xi_{\alpha}} d \omega_{\beta}(z)=\varpi_{\beta}\left(\xi_{\alpha}\right)-\varpi_{\beta}\left(\xi_{0}\right)=\delta_{\alpha \beta}, \\
\oint_{B_{\alpha}} d \omega_{\beta} & =\frac{i}{2} \oint_{\partial \mathrm{D}_{\alpha}} \partial_{n} \varpi_{\beta}(\zeta)|d \zeta|=T_{\alpha \beta},
\end{aligned}
$$

где последнее равенство определяет матрицу периодов дубля Шоттки (6.27).

Чтобы охарактеризовать форму многосвязной области, по-прежнему можно использовать гармонические моменты. Однако набор гармонических функций следует расширить, добавив функции с полюсами в каждой дырке (а не только в $\mathrm{D}_{0}$, как раньше) вместе с добавлением неоднозначных голоморфных объектов. Более конкретно, отметим точки $z_{\alpha} \in \mathrm{D}_{\alpha}$ в каждой дырке (см. рис. 18 , без потери общности удобно выбрать $\left.z_{0}=0\right)$ и рассмотрим однозначные голоморфные функции в $\mathrm{D}^{\mathrm{c}}$ вида $\left(z-z_{\alpha}\right)^{-k}$, а также гармонические функции $\ln \left|1-z_{\alpha} / z\right|^{2}$, у которых голоморфная часть многозначна. Аргументы, практически совпадающие с теми, которые были использованы при обсуждении односвязного случая, показывают [31], что параметры

$$
M_{n, \alpha}=-\frac{1}{\pi} \int_{\mathrm{D}^{\mathrm{c}}}\left(z-z_{\alpha}\right)^{-n} d^{2} z, \quad \alpha=0,1, \ldots, g, \quad n \geqslant 1,
$$

вместе с комплексно-сопряженными соотношениями

$$
\phi_{\alpha}=-\frac{1}{\pi} \int_{\mathrm{D}^{\mathrm{c}}} \ln \left|1-\frac{z_{\alpha}}{z}\right|^{2} d^{2} z=\Phi(0)-\Phi\left(z_{\alpha}\right)-\left|z_{\alpha}\right|^{2}, \quad \alpha=1,2, \ldots, g,
$$

и параметром $t_{0} \propto$ Area(D), определенным по-прежнему формулой (6.6), однозначно задают область $\mathrm{D}^{\mathrm{c}}$, т.е. любая деформация, сохраняющая все эти параметры, тривиальна. Существенным шагом является переход от переменных $M_{n, \alpha}$ к переменным $\tau_{k}[31]$, которые являются конечными линейными комбинациями $M_{n, \alpha}$ или моментами

$$
\tau_{0}=t_{0}, \quad \tau_{k}=\frac{1}{2 \pi i} \oint_{\partial \mathrm{D}} \mathrm{a}_{k}(z) \bar{z} d z=-\frac{1}{\pi} \int_{\mathrm{D}^{c}} \mathrm{a}_{k}(z) d^{2} z, \quad k>0,
$$

по отношению к некоторому базису функций Кричевера-Новикова (индексы $\alpha$ и $\beta$ следует понимать по модулю $g+1)$ :

$$
\begin{gathered}
\mathrm{a}_{m(g+1)+\alpha}(z)=\prod_{\beta=0}^{g}\left(z-z_{\beta}\right)^{-m} \prod_{\beta=0}^{\alpha-1}\left(z-z_{\beta}\right)^{-1}, \\
\mathrm{a}_{k}(z) \underset{z \rightarrow \infty}{=} z^{-k}+O\left(z^{-k-1}\right) .
\end{gathered}
$$


Любая аналитическая функция на $\mathrm{D}^{\mathrm{c}}$, обращающаяся в нуль на бесконечности, может быть представлена как линейная комбинация функций $\mathrm{a}_{k}$, сходящаяся в областях, где $\left|\prod_{\beta=0}^{g}\left(z-z_{\beta}\right)\right|>$ const $^{21)}$.

Параметры $\tau_{k}, \phi_{\alpha}$ можно использовать в качестве локальных координат в пространстве многосвязных областей, как и в односвязном случае (детали доказательства можно найти в работе [31], см. также приложение 2). Вместо величин $\phi_{\alpha}$ можно пользоваться уже введенными числами заполнения или площадями дырок

$$
S_{\alpha}=\frac{\operatorname{Area}\left(\mathrm{D}_{\alpha}\right)}{\pi}=\frac{1}{\pi} \int_{\mathrm{D}_{\alpha}} d^{2} z=\frac{1}{2 \pi i} \oint_{\partial \mathrm{D}_{\alpha}} \bar{z} d z=\frac{1}{2 \pi i} \oint_{B_{\alpha}} \tilde{z} d z, \quad \alpha=1,2, \ldots, g,
$$

где мы опять воспользовались функцией Шварца $\tilde{z}(z)=\bar{z}, \quad z \in \partial \mathrm{D}$, в некоторой полосе в окрестности всех границ. Заметим, что в обозначениях этого раздела переменные $S_{\alpha}$ выражаются естественным образом через $B_{\alpha}$-периоды производящего дифференциала, в отличие от чаще используемого соглашения (5.15). Это связано с тем, что для дубля Шоттки удобно пользоваться каноническими дифференциалами (6.30), непосредственно связанными с гармонической мерой и нормированными на А-циклы, показанные на рис. 19. При другом выборе получающиеся тау-функции будут связаны друг с другом преобразованием дуальности, которое уже упоминалось выше и которое будет подробно обсуждаться в п. 6.5.

В то же время переменные $\phi_{\alpha}$ не имеют явного геометрического смысла на дубле Шоттки, и вместо них естественно ввести новые величины $\Pi_{\alpha}$ (опять совпадение обозначений с введенными выше множителями Лагранжа не случайно), которые в работе [31] были названы виртуальнъмми А-периодами дифференциала $\tilde{z}(z) d z$ на дубле Шоттки, поскольку они совпадают с реальными А-периодами всегда, когда последние строго определены. Следуя [31], рассмотрим базис дифференциалов $d \mathrm{~b}_{k}$, удовлетворяющих условиям ортогональности

$$
\frac{1}{2 \pi i} \oint_{\partial \mathrm{D}} \mathrm{a}_{k} d \mathrm{~b}_{k^{\prime}}=\delta_{k, k^{\prime}}
$$

для всех целых чисел $k, k^{\prime} \in \mathbb{Z}$. Явно они могут быть представлены формулами

$$
d \mathrm{~b}_{m(g+1)+\alpha}=\frac{d z}{z-z_{g}} \prod_{\beta=0}^{g}\left(z-z_{\beta}\right)^{m} \prod_{\beta=0}^{\alpha-1}\left(z-z_{\beta-1}\right),
$$

в которых мы отождествили $z_{-1} \equiv z_{g}$. Некоторые свойства функций (6.34) и дуальных к ним дифференциалов (6.37) можно найти в приложении 2. Теперь можно ввести дифференциал на $\Sigma$ с единственным полюсом в точке $\infty$ на верхнем листе, в окрестности которой он имеет вид

$$
d \widetilde{\Omega}_{k}(z)=d \mathbf{b}_{k}(z)+O\left(z^{-2}\right) d z
$$

21) В случае одной дырки $g=0$ формулы (6.34) возвращают нас к базису, использованному в п. 6.1, $\mathrm{a}_{k}=z^{-k}$. Заметим также, что всегда верно $\mathrm{a}_{0}=1, \mathrm{a}_{1}=1 / z$, а следовательно, $\tau_{0}=t_{0}$ и $\tau_{1}=M_{1,0}=t_{1}$. 
а кроме того, его А-периоды обращаются в нуль:

$$
\oint_{A_{\alpha}} d \widetilde{\Omega}_{k}=0
$$

т.е. мы ввели канонически нормированный мероморфный дифференциал с главной частью (6.38). Нормальное смещение границы, задаваемое вещественной и мнимой частями нормальной производной $\partial_{n} \Omega_{k}$, определяет комплексное тангенциальное к пространству многосвязных областей векторное поле (частную производную при постоянных $\Pi_{\alpha}$ )

$$
\partial_{\tau_{k}}^{\Pi}=\partial_{\tau_{k}}^{\phi}-\sum_{\alpha} \mathrm{b}_{k}\left(z_{\alpha}\right) \partial_{\alpha}^{\phi}
$$

где

$$
\mathrm{b}_{k}(z)=\int_{0}^{z} d \mathrm{~b}_{k}
$$

- полином степени $k$. Эти векторные поля сохраняют формальные переменные

$$
\Pi_{\alpha}=\phi_{\alpha}+2 \operatorname{Re} \sum_{k} \mathrm{~b}_{k}\left(z_{\alpha}\right) \tau_{k} .
$$

Ряд в правой части, вообще говоря, расходится, но в случаях, когда функция Шварца может быть продолжена мероморфно на дубль $\Sigma$, ряд сходится, а полученное выражение совпадает с $A_{\alpha}$-периодом продолжения дифференциала $\tilde{z} d z$ (см. ниже формулу (6.71)).

Как и в односвязном случае, можно ввести элементарные деформации

$$
\begin{aligned}
& \delta_{z}: \quad \delta n(\xi)=-\frac{\epsilon}{2} \partial_{n} G(z, \xi), \quad z \in \mathrm{D}^{\mathrm{c}}, \\
& \delta^{(\alpha)}: \quad \delta n(\xi)=-\frac{\epsilon}{2} \partial_{n} \varpi_{\alpha}(\xi), \quad \alpha=1,2, \ldots, g,
\end{aligned}
$$

где $\varpi_{\alpha}(z)$ является гармонической мерой компоненты границы $\partial \mathrm{D}_{\alpha}(\mathrm{cm} .(6.26))$. Тогда вариации локальных координат относительно элементарных деформаций имеют вид

$$
\delta_{z} \tau_{k}=\epsilon \mathrm{a}_{k}(z), \quad \delta_{z} \phi_{\alpha}=\epsilon \ln \left|1-\frac{z_{\alpha}}{z}\right|^{2}, \quad \delta^{(\alpha)} \tau_{k}=0, \quad \delta^{(\alpha)} \phi_{\beta}=-2 \epsilon \delta_{\alpha \beta},
$$

поскольку

$$
\delta_{z} \int_{\mathrm{D}^{\mathrm{c}}} f(\zeta) d^{2} \zeta=\frac{\epsilon}{2} \oint_{\partial \mathrm{D}^{\mathrm{c}}} f(\zeta) \partial_{n} G(z, \zeta)|d \zeta|=-\epsilon \pi f(z)
$$

для любой гармонической функции $f$ на $\mathrm{D}^{\mathrm{c}}$, а кроме того,

$$
\delta^{(\alpha)} \int_{\mathrm{D}^{\mathrm{c}}} f(\zeta) d^{2} \zeta=\frac{\epsilon}{2} \oint_{\partial \mathrm{D}^{\mathrm{c}}} f \partial_{n} \varpi_{\alpha}|d \zeta|=-\frac{\epsilon}{2} \oint_{\partial \mathrm{D}_{\alpha}} \partial_{n} f|d \zeta|=-i \epsilon \oint_{\partial \mathrm{D}_{\alpha}} \partial_{\zeta} f d \zeta,
$$

что приводит к формулам (6.44) для конкретных примеров функций $f$. Вариации переменных $\Pi_{\alpha}$ (в случае, когда они хорошо определены) тогда равны

$$
\delta_{z} \Pi_{\alpha}=0, \quad \delta^{(\alpha)} \Pi_{\beta}=-2 \epsilon \delta_{\alpha \beta},
$$


и для любого функционала $X$ на пространстве многосвязных областей выполняются следующие равенства:

$$
\delta_{z} X=\epsilon \nabla(z) X, \quad \delta^{(\alpha)} X=-2 \epsilon \partial_{\alpha}^{\phi} X=-2 \epsilon \partial_{\alpha}^{\Pi} X .
$$

Дифференциальный оператор $\nabla(z)$ теперь задается формулой

$$
\nabla(z)=\partial_{\tau_{0}}^{\Pi}+\sum_{k \geqslant 1}\left(\mathrm{a}_{k}(z) \partial_{\tau_{k}}^{\Pi}+\overline{\mathrm{a}_{k}(z)} \partial_{\bar{\tau}_{k}}^{\Pi}\right) .
$$

Функционал $X$ можно рассматривать как функцию $X=X^{\phi}\left(\phi_{\alpha}, \tau_{k}\right)$ на пространстве с локальными координатами $\phi_{\alpha}, \tau_{k}$, или как функцию $X=X^{\Pi}\left(\Pi_{\alpha}, \tau_{k}\right)$ на пространстве с локальными координатами $\Pi_{\alpha}, \tau_{k}$ (подчеркнем, что хотя переменные $\Pi_{\alpha}$ формальны, их вариации при элементарных деформациях и векторные поля $\partial_{\tau_{k}}^{\Pi}$ всегда хорошо определены).

Пусть теперь $K(z, \zeta) d \zeta$ представляет собой единственный мероморфный абелев дифференциал третьего рода (4.5), (4.6) на дубле Шоттки $\Sigma$ с простыми полюсами в точках $z$ и $\infty$ на верхнем листе и вычетами \pm 1 . Тогда

$$
2 \partial_{\zeta} G(z, \zeta) d \zeta-2 \partial_{\zeta} G(\infty, \zeta) d \zeta=K(z, \zeta) d \zeta+K(\bar{z}, \zeta) d \zeta
$$

и дифференциал $d \widetilde{\Omega}_{k}(\zeta)(6.38),(6.39)$ может быть представлен в виде

$$
d \widetilde{\Omega}_{k}(\zeta)=\frac{d \zeta}{2 \pi i} \oint_{\infty} K(u, \zeta) d \mathrm{~b}_{k}(u)
$$

где интегрирование по $u$ проводится по большому кругу вокруг бесконечности. Используя формулы суммирования (П.5) (см. приложение 2), получим

$$
-\sum_{k \geqslant 1} \mathrm{a}_{k}(z) d \widetilde{\Omega}_{k}(\zeta)=\frac{d \zeta}{2 \pi i} \oint_{\infty} \frac{K(u, \zeta) d u}{u-z}=K(z, \zeta) d \zeta .
$$

Поэтому для функции Грина существует разложение

$$
2 \partial_{\zeta} G(z, \zeta) d \zeta=d \Omega_{0}(\zeta)-\sum_{k \geqslant 1}\left(\mathrm{a}_{k}(z) d \widetilde{\Omega}_{k}(\zeta)+\text { c.c. }\right)
$$

где комплексно-сопряженный дифференциал $\overline{d \widetilde{\Omega}_{k}}$ представляет собой единственный мероморфный дифференциал на $\Sigma$ с полюсом бесконечности $\bar{\infty}$ на нижнем листе, главной частью $-d \overline{\mathrm{b}_{k}(z)}$ и нулевыми $\mathbf{A}$-периодами.

6.3. Квазиклассическая тау-функция многосвязной области. Применяя формулы (6.2), (6.28), (6.29), легко получить вариации функции Грина, гармонической меры и матрицы периодов при элементарных деформациях:

$$
\begin{aligned}
\delta_{z_{1}} G\left(z_{2}, z_{3}\right) & =\delta_{z_{2}} G\left(z_{3}, z_{1}\right)=\delta_{z_{3}} G\left(z_{1}, z_{2}\right), \\
\delta_{z_{1}} \varpi_{\alpha}\left(z_{2}\right) & =\delta^{(\alpha)} G\left(z_{1}, z_{2}\right)=\delta_{z_{2}} \varpi_{\alpha}\left(z_{1}\right), \\
\delta^{(\alpha)} \varpi_{\beta}(z) & =\delta^{(\beta)} \varpi_{\alpha}(z), \\
\delta_{z} T_{\alpha \beta} & =i \pi \delta^{(\alpha)} \varpi_{\beta}(z), \\
\delta^{(\alpha)} T_{\beta \gamma} & =\delta^{(\beta)} T_{\gamma \alpha}=\delta^{(\gamma)} T_{\alpha \beta} .
\end{aligned}
$$


Из (6.48) следует, что формулы (6.54) могут быть переписаны в терминах дифференциальных операторов $\nabla(z)$ и $\partial_{\alpha}=\partial / \partial \phi_{\alpha}=\partial / \partial \Pi_{\alpha}$ :

$$
\begin{aligned}
\nabla\left(z_{1}\right) G\left(z_{2}, z_{3}\right) & =\nabla\left(z_{2}\right) G\left(z_{3}, z_{1}\right)=\nabla\left(z_{3}\right) G\left(z_{1}, z_{2}\right), \\
\nabla\left(z_{1}\right) \varpi_{\alpha}\left(z_{2}\right) & =-2 \partial_{\alpha} G\left(z_{1}, z_{2}\right), \\
\partial_{\alpha} \varpi_{\beta}(z) & =\partial_{\beta} \varpi_{\alpha}(z), \\
\nabla(z) T_{\alpha \beta} & =-2 \pi i \partial_{\alpha} \varpi_{\beta}(z), \\
\partial_{\alpha} T_{\beta \gamma} & =\partial_{\beta} T_{\gamma \alpha}=\partial_{\gamma} T_{\alpha \beta} .
\end{aligned}
$$

Эти условия интегрируемости обобщают формулы (6.20) на многосвязный случай; первая из них буквально совпадает с (6.20), в то время как остальные расширяют условие симметрии производных на гармоническую меру и матрицу периодов.

Выражения (6.55) можно понимать как условие совместности для бесконечной иерархии дифференциальных уравнений. Из них следует, что существует функция $\mathcal{F}_{\mathrm{D}}=\mathcal{F}_{\mathrm{D}}\left(\Pi_{\alpha}, \tau\right)$ такая, что

$$
\begin{aligned}
G\left(z_{1}, z_{2}\right) & =\ln \left|z_{1}^{-1}-z_{2}{ }^{-1}\right|+\frac{1}{2} \nabla\left(z_{1}\right) \nabla\left(z_{2}\right) \mathcal{F}_{\mathrm{D}} \\
\varpi_{\alpha}(z) & =-\partial_{\alpha} \nabla(z) \mathcal{F}_{\mathrm{D}} \\
T_{\alpha \beta} & =2 \pi i \partial_{\alpha} \partial_{\beta} \mathcal{F}_{\mathrm{D}}
\end{aligned}
$$

Функция $\mathcal{F}_{\mathrm{D}} F$ является логарифмом квазиклассической тау-функции многосвязных областей, и она будет отождествлена в п. 6.5 со свободной энергией многокомпонентных решений матричной модели (2.5) после преобразования дуальности.

Положим опять $\tilde{\Phi}(z)=\nabla(z) \mathcal{F}_{\mathrm{D}}$, тогда уравнения $(6.56)$ определяют $\tilde{\Phi}(z)$ для $z \in \mathrm{D}^{\mathrm{c}}$ по его вариациям относительно элементарных деформаций:

$$
\begin{aligned}
\delta_{\zeta} \tilde{\Phi}(z) & =-2 \epsilon \ln \left|\zeta^{-1}-z^{-1}\right|+2 \epsilon G(\zeta, z), \\
\delta^{(\alpha)} \tilde{\Phi}(z) & =2 \epsilon \varpi_{\alpha}(z) .
\end{aligned}
$$

Действительно, используя (6.43), для вариации формулы

$$
\tilde{\Phi}(z)=-\frac{2}{\pi} \int_{\mathrm{D}} \ln \left|z^{-1}-\zeta^{-1}\right| d^{2} \zeta=\Phi(z)-\Phi(0)+\tau_{0} \ln |z|^{2},
$$

буквально совпадающей с определением (6.23), если понимать D как объединение всех областей $\mathrm{D}_{\alpha}$, получаем

$$
\begin{gathered}
\delta_{\zeta}\left(-\frac{2}{\pi} \int_{\mathrm{D}} \ln \left|z^{-1}-z^{\prime-1}\right| d^{2} z^{\prime}\right)=\frac{\epsilon}{\pi} \oint_{\partial \mathrm{D}^{\mathrm{c}}} \partial_{n} G(\zeta, \xi) \ln \left|z^{-1}-\xi^{-1}\right||d \xi|= \\
=\frac{\epsilon}{\pi} \oint_{\partial \mathrm{D}^{\mathrm{c}}} \partial_{n} G(\zeta, \xi)\left(\ln \left|z^{-1}-\xi^{-1}\right|-G(z, \xi)\right)|d \xi|= \\
=-2 \epsilon \ln \left|\zeta^{-1}-z^{-1}\right|+2 \epsilon G(\zeta, z) .
\end{gathered}
$$


Аналогично для $z \in \mathrm{D}^{\mathrm{c}}$ имеем

$$
\begin{gathered}
\delta^{(\alpha)}\left(-\frac{2}{\pi} \int_{\mathrm{D}} \ln \left|z^{-1}-\zeta^{-1}\right| d^{2} \zeta\right)=\frac{\epsilon}{\pi} \oint_{\partial \mathrm{D}^{\mathrm{c}}} \partial_{n} \varpi_{\alpha}(\xi) \ln \left|z^{-1}-\xi^{-1}\right||d \xi|= \\
\quad=\frac{\epsilon}{\pi} \oint_{\partial \mathrm{D}^{\mathrm{c}}} \partial_{n} \varpi_{\alpha}(\xi)\left(\ln \left|z^{-1}-\xi^{-1}\right|-G(z, \xi)\right)|d \xi|= \\
=-\frac{\epsilon}{\pi} \oint_{\partial \mathrm{D}_{\alpha}} \partial_{n}\left(\ln \left|z^{-1}-\xi^{-1}\right|-G(z, \xi)\right)|d \xi|=2 \epsilon \varpi_{\alpha}(z) .
\end{gathered}
$$

Проведя то же самое вычисление при $z \in \mathrm{D}$, имеем

$$
\delta^{(\alpha)} \tilde{\Phi}(z)= \begin{cases}0, & z \in \mathrm{D}_{0} \\ 2 \epsilon \delta_{\alpha \beta}, & z \in \mathrm{D}_{\beta}, \beta=1,2, \ldots, g\end{cases}
$$

Коэффициенты разложения $\tilde{\Phi}$ в бесконечности определяют дуальные моменты $\nu_{k}$ :

$$
\nabla(z) \mathcal{F}_{\mathrm{D}}=\tilde{\Phi}(z)=-\frac{2}{\pi} \int_{\mathrm{D}} \ln \left|z^{-1}-\zeta^{-1}\right| d^{2} \zeta=v_{0}+2 \operatorname{Re} \sum_{k>0} \nu_{k} \mathrm{a}_{k}(z),
$$

которые являются моментами объединения внутренности дырок по отношению к дуальному базису

$$
\nu_{k}=\frac{1}{\pi} \int_{\mathrm{D}} \mathrm{b}_{k}(z) d^{2} z
$$

Из (6.62) следует, что

$$
\nu_{k}=\partial_{\tau_{k}}^{\Pi} \mathcal{F}_{\mathrm{D}},
$$

и те же самые аргументы показывают, что производные

$$
S_{\alpha}=-\partial_{\alpha} \mathcal{F}_{\mathrm{D}}
$$

совпадают с площадями дырок (6.35). Действительно, с учетом (6.61) имеем

$$
\begin{aligned}
\delta^{(\alpha)} \mathcal{F}_{\mathrm{D}} & =\frac{1}{2 \pi} \delta^{(\alpha)}\left(\int_{\mathrm{D}} \tilde{\Phi}(z) d^{2} z\right)=-\frac{\epsilon}{4 \pi} \oint_{\partial \mathrm{D}^{\mathrm{c}}} \partial_{n} \varpi_{\alpha}(\xi) \tilde{\Phi}(\xi)|d \xi|+\frac{1}{2 \pi} \int_{\mathrm{D}} \delta^{(\alpha)} \tilde{\Phi}(\zeta) d^{2} \zeta= \\
& =\frac{\epsilon}{4 \pi} \oint_{\partial \mathrm{D}_{\alpha}} \partial_{n} \tilde{\Phi}(\xi)|d \xi|+\frac{\epsilon}{\pi} \int_{\mathrm{D}_{\alpha}} d^{2} \zeta=-\frac{\epsilon}{4 \pi} \int_{\mathrm{D}_{\alpha}} \Delta \tilde{\Phi} d^{2} \zeta+\epsilon S_{\alpha}=2 \epsilon S_{\alpha} .
\end{aligned}
$$

Интегральное представление функции $\mathcal{F}_{\mathrm{D}}$ находится аналогично с помощью его вариаций, определяемых из формул (6.62), а результат выражается той же самой формулой (6.25), как и в односвязном случае, если считать, что $\mathrm{D}=\bigcup_{\alpha=0}^{g} \mathrm{D}_{\alpha}$ представляет теперь объединение всех областей $\mathrm{D}_{\alpha}$.

6.4. Полиномиальные потенциалы и алгебраические области. Области называются алгебраическими, если функция Шварца $\tilde{z}(z)$ может быть мероморфно продолжена на риманову поверхность $\Sigma$ с антиголоморфной инволюцией, тогда $\Sigma$ можно естественным образом представить в виде двух “половинок" (называемых 
верхним и нижним листами), переходящих друг в друга при этой инволюции. Область $\mathrm{D}^{\mathrm{c}}$ является алгебраической тогда и только тогда, когда интегралы Коши

$$
C^{\alpha}(z)=\frac{1}{2 \pi i} \oint_{\partial \mathrm{D}} \frac{\bar{\zeta} d \zeta}{\zeta-z}, \quad z \in \mathrm{D}_{\alpha}
$$

продолжаются до рациональной мероморфной функции $\mathcal{S}(z)$ (одной и той же при всех $\alpha$ !) на всей комплексной плоскости $\mathbb{C}$ с отмеченной точкой в бесконечности. Равенство $\tilde{z}(z)=\mathcal{S}(z)-C^{-}(z)$, выполняющееся по определению для $z \in \partial \mathrm{D}^{\mathrm{c}}$, может быть использовано для аналитического продолжения функции Шварца. Поскольку функция $C^{-}(z)$ аналитична в $\mathrm{D}^{\mathrm{c}}$, функции $\mathcal{S}(z)$ и $\tilde{z}(z)$ имеют совпадающие сингулярные части в их полюсах в области $\mathrm{D}^{\mathrm{c}}$. Функцию $\tilde{z}(z)$ можно при этом рассматривать как функцию на дубле Шоттки, продолжая ее на нижний лист как $\bar{z}$.

Удобно также ввести абелев интеграл

$$
\mathrm{b}(z)=\int_{0}^{z} \mathcal{S}(z) d z
$$

который многозначен, если у функции $\mathcal{S}(z)$ есть простые полюсы (чтобы выделить однозначную ветвь, нужно провести разрезы из бесконечности во все простые полюсы $\mathcal{S}(z))$. В окрестности точек $z_{\alpha} \in \mathrm{D}_{\alpha}($ см. (6.34), (6.37)) имеем

$$
\mathcal{S}(z) d z=\sum_{k \geqslant 1} \tau_{k} d \mathrm{~b}_{k}(z), \quad \mathrm{b}(z)=\sum_{k \geqslant 1} \tau_{k} \mathrm{~b}_{k}(z) .
$$

Формула (6.42) показывает, что для алгебраических областей переменные $\Pi_{\alpha}$, введенные в общем случае как формальные объекты, являются хорошо определенными величинами, равными А-периодам дифференциала $\tilde{z}(z) d z$ на дубле Шоттки $\Sigma$. Действительно, пользуясь тем, что $C^{0}(z)$ и $C^{\alpha}(z)$ представляют собой ограничения одной $и$ той же функции $\mathcal{S}(z)$, можно использовать представление (П.8) (см. приложение 2) и переписать его в виде

$$
\phi_{\alpha}=-2 \operatorname{Re}\left(\int_{0}^{z_{\alpha}} \mathcal{S}(z) d z+\int_{\xi_{0}}^{\xi_{\alpha}}\left(\bar{z}+C^{-}(z)-\mathcal{S}(z)\right) d z\right) .
$$

Комбинируя это равенство с определением $\Pi_{\alpha}(6.42)$, получаем

$$
\Pi_{\alpha}=2 \operatorname{Re} \int_{\xi_{0}}^{\xi_{\alpha}}(\tilde{z}(z)-\bar{z}) d z=\int_{\xi_{0}}^{\xi_{\alpha}}(\tilde{z}(z) d z-\bar{z} d \overline{\tilde{z}(z)})=\oint_{A_{\alpha}} \tilde{z} d z
$$

В качестве примера алгебраических областей полезно рассмотреть случай конечного числа ненулевых моментов $\tau_{k}$, т.е. $\tau_{k}=0$ при $k>n+1$, отвечающий двухматричной модели с полиномиальным потенциалом (2.4). Тогда $\tilde{z}(z)$ продолжается до мероморфной функции на $\Sigma$ с полюсом порядка $n$ в точке $\infty$ и простым полюсом в $\bar{\infty}$. Функция $z$ продолжается на нижний лист дубля Шоттки как $\overline{\tilde{z}(z)}$, и у нее имеется простой полюс в точке $\infty$, а в $\bar{\infty}-$ полюс порядка $n$. Для такой области $\mathrm{D}^{c}$ моменты по отношению к лорановскому базису (6.4), как и в односвязном случае, 
совпадают с коэффициентами разложения функции Шварца около $\infty$ :

$$
\tilde{z}(z)=\sum_{k=1}^{n+1} k t_{k} z^{k-1}+O\left(z^{-1}\right), \quad z \rightarrow \infty,
$$

что буквально воспроизводит формулу (5.18). Нормальное смещение границы алгебраической области, которое меняет переменную $t_{k}$, сохраняя все остальные моменты (а также $\left.\Pi_{\alpha}\right)$ без изменений, определяется нормальной производной функции $2 \operatorname{Re} \int^{z} d \Omega_{k}$, где $d \Omega_{k}$ является нормированным абелевым мероморфным дифференциалом второго рода (4.4) на $\Sigma$ с единственным полюсом в точке $\infty$ :

$$
d \Omega_{k}=d\left(z^{k}+O\left(z^{-1}\right)\right), \quad \oint_{A_{\alpha}} d \Omega_{k}=0 .
$$

Пусть также $d \Omega_{0}$ представляет собой абелев дифференциал третьего рода (4.5), (4.6) на $\Sigma$ с полюсами в двух бесконечностях $\infty$ и $\bar{\infty}$, тогда его абелев интеграл

$$
\ln w(z)=\int_{\xi_{0}}^{z} d \Omega_{0}
$$

определяет в окрестности точки $\infty$ функцию $w(z)$ с простым полюсом в бесконечности. Зависимость обратной функции $z(w)$ от переменных $t_{k}$ описывается уравнениями Уизема для иерархии двумеризованной цепочки Тоды следующего вида:

$$
\partial_{t_{k}}^{\Pi} z(w)=\left\{\Omega_{k}(w), z(w)\right\}=\frac{d \Omega_{k}(w)}{d \ln w} \partial_{t_{0}} z(w)-\partial_{t_{0}} \Omega_{k}(w) \frac{d z}{d \ln w} .
$$

В этом случае можно также выписать условие квазиоднородности [22] для квазиклассической тау-функции, которое принимает вид

$$
2 \mathcal{F}_{\mathrm{D}}=-\frac{1}{2} \tau_{0}^{2}+\tau_{0} v_{0}+\frac{1}{2} \sum_{k \geqslant 1}(2-k)\left(\tau_{k} \nu_{k}+\bar{\tau}_{k} \bar{\nu}_{k}\right)-\sum_{\alpha=1}^{g} \Pi_{\alpha} S_{\alpha} .
$$

Алгебраические области более общего вида отвечают универсальной иерархии Уизема [22], обсуждение этого вопроса можно найти в работе [31].

\section{5. Преобразование дуальности и свободная энергия двухматричной} модели. Переход от $\Pi_{\alpha}$ к $S_{\alpha}$ является частным случаем преобразований дуальности (4.17), который эквивалентен перестановке А- и В-циклов на римановой поверхности $\Sigma$ (см. рис. 3). Для квазиклассической тау-функции, зависящей также от дополнительных переменных $\tau$, это преобразование сводится к частичному преобразованию Лежандра $\mathcal{F}_{\mathrm{D}}\left(\Pi_{\alpha}, \boldsymbol{\tau}\right) \rightarrow \mathcal{F}\left(S_{\alpha}, \boldsymbol{\tau}\right)$, где

$$
\mathcal{F}_{\mathrm{D}}=\mathcal{F}+\sum_{\alpha=1}^{g} \Pi_{\alpha} S_{\alpha}
$$

Функция $\mathcal{F}$ представляет собой дуальный препотенциал по отношению к тау-функции задачи Дирихле, она является решением модифицированной задачи Дирихле, и 
ее можно отождествить со свободной энергией двухматричной модели в планарном пределе больших $N$, когда носитель собственных значений состоит из нескольких несвязных областей.

Основные свойства $\mathcal{F}$ вытекают непосредственно из свойств $\mathcal{F}_{\mathrm{D}}$. Согласно $(6.64)$, (6.65) имеем

$$
d \mathcal{F}_{\mathrm{D}}=-\sum_{\alpha} S_{\alpha} d \Pi_{\alpha}+\sum_{k} \nu_{k} d \tau_{k}
$$

(для краткости индекс $k$ предполагается пробегающим все целые значения, т.е. $\left.\tau_{-k} \equiv \bar{\tau}_{k}\right)$, так что

$$
d \mathcal{F}=\sum_{\alpha} \Pi_{\alpha} d S_{\alpha}+\sum_{k} \nu_{k} d \tau_{k}
$$

Это дает ответ для первых производных:

$$
\Pi_{\alpha}=\frac{\partial \mathcal{F}}{\partial S_{\alpha}}, \quad \nu_{k}=\frac{\partial \mathcal{F}}{\partial \tau_{k}} .
$$

Вторые производные преобразуются следующим образом (см., например, [24]): положим

$$
\mathcal{F}_{\mathrm{D} \alpha \beta}=\frac{\partial^{2} \mathcal{F}_{\mathrm{D}}}{\partial \Pi_{\alpha} \partial \Pi_{\beta}}, \quad \mathcal{F}_{\mathrm{D} \alpha k}=\frac{\partial^{2} \mathcal{F}_{\mathrm{D}}}{\partial \Pi_{\alpha} \partial \tau_{k}}, \quad \mathcal{F}_{\mathrm{D} i k}=\frac{\partial^{2} \mathcal{F}_{\mathrm{D}}}{\partial \tau_{i} \partial \tau_{k}}
$$

и аналогично для $\mathcal{F}$, тогда

$$
\begin{aligned}
& \mathcal{F}_{\mathrm{D} \alpha \beta}=-\left(\mathcal{F}^{-1}\right)_{\alpha \beta}, \quad \mathcal{F}_{\mathrm{D} \alpha k}=\sum_{\gamma=1}^{g}\left(\mathcal{F}^{-1}\right)_{\alpha \gamma} \mathcal{F}_{\gamma k}, \\
& \mathcal{F}_{\mathrm{D} i k}=\mathcal{F}_{i k}-\sum_{\gamma, \gamma^{\prime}=1}^{g} \mathcal{F}_{i \gamma}\left(\mathcal{F}^{-1}\right)_{\gamma \gamma^{\prime}} \mathcal{F}_{\gamma^{\prime} k}
\end{aligned}
$$

где $\left(\mathcal{F}^{-1}\right)_{\alpha \beta}$ обозначает матричный элемент матрицы, обратной к матрице $\mathcal{F}_{\alpha \beta}$ размера $g \times g$.

Используя эти формулы, легко видеть, что основные свойства (6.56) тау-функции наследуются дуальной тау-функцией в следующем виде:

$$
\begin{gathered}
\widetilde{G}(z, \zeta)=\ln \left|z^{-1}-\zeta^{-1}\right|+\frac{1}{2} \nabla(z) \nabla(\zeta) \mathcal{F}, \\
2 \pi i \widetilde{\varpi}_{\alpha}(z)=-\partial_{S_{\alpha}} \nabla(z) \mathcal{F}, \\
2 \pi i \widetilde{T}_{\alpha \beta}=\frac{\partial^{2} \mathcal{F}}{\partial S_{\alpha} \partial S_{\beta}},
\end{gathered}
$$

где производные по $\tau_{k}$ в $\nabla(z)$ берутся при фиксированных $S_{\alpha}$. Объекты в левых частях этих равенств представляют собой

$$
\begin{gathered}
\widetilde{G}(z, \zeta)=G(z, \zeta)+i \pi \sum_{\alpha, \beta=1}^{g} \omega_{\alpha}(z) \widetilde{T}_{\alpha \beta} \omega_{\beta}(\zeta), \\
\widetilde{\omega}_{\alpha}(z)=\sum_{\beta=1}^{g} \widetilde{T}_{\alpha \beta} \omega_{\beta}(z), \quad \widetilde{T}=-T^{-1} .
\end{gathered}
$$


Функция $\widetilde{G}$ является функцией Грина модифицированной задачи Дирихле (см. [31]). Матрица $\widetilde{T}$ является матрицей А-периодов голоморфных дифференциалов $d \widetilde{\omega}_{\alpha}$ на дубле $\Sigma$ (таких, что $\left.\widetilde{\varpi}_{\alpha}(z)=\widetilde{\omega}_{\alpha}(z)+\overline{\widetilde{\omega}}_{\alpha}(z)\right)$, нормированных на В-циклы:

$$
\oint_{B_{\alpha}} d \widetilde{\omega}_{\beta}=-\delta_{\alpha \beta}, \quad \oint_{A_{\alpha}} d \widetilde{\omega}_{\beta}=\widetilde{T}_{\alpha \beta},
$$

т.е. более точно замена циклов при преобразовании дуальности представляет собой замену $A_{\alpha} \rightarrow B_{\alpha}, B_{\alpha} \rightarrow-A_{\alpha}$.

Важный факт заключается в том, что простым изменением масштаба независимых переменных можно переписать соотношения (6.81) на функцию $\mathcal{F}$ в точности в виде (6.56), так что они будут отличаться только обозначениями. Поэтому в дальнейшем в работе [32] мы не будем даже различать эти два случая при изучении явных дифференциальных уравнений, которым удовлетворяют квазиклассические тау-функции матричных моделей.

\section{7. ЗАКЛЮЧЕНИЕ}

Во второй части данной работы [32] мы рассмотрим некоторые примеры приложений изученных выше методов и исследуем похожую геометрическую картину, возникающую в случае многомерных калибровочных теорий и АдС/KТП-соответствия. Мы также собираемся кратко обсудить нерешенные задачи и выяснить, как эта картина может выглядеть вне рамок квазиклассического приближения.

\section{Рациональные вырождения}

ПРИЛОЖКЕНИЕ 1

Подставляя формулы (5.35) в (5.10) и вычисляя вычеты, находим, что выражения

$$
R_{l}[F]=\operatorname{res}\left(\frac{d w}{w} w^{l} F(z(w), \tilde{z}(w))\right)=0
$$

для $l=-n(n+1), \ldots, n(n+1)$ образуют треугольную систему уравнений на коэффициенты $f_{i j}$. Это означает, что каждое из уравнений (П.1) линейно по одному из коэффициентов, и они могут быть разрешены шаг за шагом, начиная с концов цепочки.

Для кубического потенциала $(n=2)$ решение имеет вид

$$
\begin{aligned}
& a=-\frac{r^{2}}{u_{2}}, \\
& b=\frac{u_{1} r}{u_{2}}-2 \bar{u}_{0}, \\
& c=-\frac{u_{1} r \bar{u}_{0}}{u_{2}}+\bar{u}_{0}^{2}-2 r \bar{u}_{1}+3 \frac{r^{2} u_{0}}{u_{2}}-\frac{r^{3} \bar{u}_{1}}{\bar{u}_{2} u_{2}}, \\
& f=r^{2}-2 u_{2} \bar{u}_{2}+4 u_{0} \bar{u}_{0}+\frac{r^{4}}{\bar{u}_{2} u_{2}}-u_{1} \bar{u}_{1}-2 \frac{r \bar{u}_{1} \bar{u}_{0}}{\bar{u}_{2}}+\frac{r^{2} \bar{u}_{1} u_{1}}{\bar{u}_{2} u_{2}}-2 \frac{r u_{0} u_{1}}{u_{2}},
\end{aligned}
$$




$$
\begin{aligned}
q=- & 3 \frac{r^{2} u_{0}^{2}}{u_{2}}+2 u_{2} \bar{u}_{2} \bar{u}_{0}-u_{2} \bar{u}_{1}^{2}-2 \frac{r^{2} \bar{u}_{1}^{2}}{\bar{u}_{2}}-\frac{r^{4} \bar{u}_{0}}{\bar{u}_{2} u_{2}}-3 r \bar{u}_{2} u_{1}+\bar{u}_{0} u_{1} \bar{u}_{1}+ \\
& +4 u_{0} r \bar{u}_{1}-2 \bar{u}_{0}^{2} u_{0}-\frac{r \bar{u}_{1} u_{1}^{2}}{u_{2}}-r^{2} \bar{u}_{0}+2 \frac{r u_{1} \bar{u}_{0} u_{0}}{u_{2}}+3 \frac{r^{3} u_{1}}{u_{2}}+ \\
& +\frac{r \bar{u}_{1} \bar{u}_{0}^{2}}{\bar{u}_{2}}-\frac{r^{2} \bar{u}_{0} u_{1} \bar{u}_{1}}{\bar{u}_{2} u_{2}}+2 \frac{u_{0} r^{3} \bar{u}_{1}}{\bar{u}_{2} u_{2}}, \\
h=- & \frac{r^{6}}{\bar{u}_{2} u_{2}}+\frac{r^{2} \bar{u}_{0}^{3}}{\bar{u}_{2}}+\bar{u}_{2} \bar{u}_{0} u_{1}^{2}-\frac{u_{0} r \bar{u}_{1} \bar{u}_{0}^{2}}{\bar{u}_{2}}+u_{2} u_{0} \bar{u}_{1}^{2}-3 \frac{r^{3} \bar{u}_{0} \bar{u}_{1}}{\bar{u}_{2}}- \\
& -\frac{\bar{u}_{2} u_{1}^{3} r}{u_{2}}-\frac{u_{2} r \bar{u}_{1}^{3}}{\bar{u}_{2}}+\frac{r^{2} u_{0}^{3}}{u_{2}}+2 \frac{r^{4} \bar{u}_{1} u_{1}}{\bar{u}_{2} u_{2}}+\frac{u_{0} \bar{u}_{0} \bar{u}_{1} r^{2} u_{1}}{\bar{u}_{2} u_{2}}-\frac{r^{3} u_{1} \bar{u}_{0}^{2}}{\bar{u}_{2} u_{2}}- \\
& -\frac{r^{3} \bar{u}_{1} u_{0}^{2}}{\bar{u}_{2} u_{2}}-\frac{u_{1}^{2} \bar{u}_{1}^{2} r^{2}}{\bar{u}_{2} u_{2}}+3 r^{4}+\bar{u}_{0}^{2} u_{0}^{2}+\frac{u_{1}^{2} \bar{u}_{1} u_{0} r}{u_{2}}-2 \bar{u}_{0}^{2} u_{1} r+ \\
& +2 \frac{u_{1}^{2} r^{2} \bar{u}_{0}}{u_{2}}+\frac{u_{1} \bar{u}_{1}^{2} r \bar{u}_{0}}{\bar{u}_{2}}+r^{2} \bar{u}_{0} u_{0}-u_{1} \bar{u}_{1} u_{0} \bar{u}_{0}+\frac{u_{0} \bar{u}_{0} r^{4}}{\bar{u}_{2} u_{2}}+u_{2}^{2} \bar{u}_{2}^{2}- \\
& -\frac{u_{1} r \bar{u}_{0} u_{0}^{2}}{u_{2}}-2 r \bar{u}_{1} u_{0}^{2}+2 \frac{u_{0} r^{2} \bar{u}_{1}^{2}}{\bar{u}_{2}}-3 \frac{r^{3} u_{0} u_{1}}{u_{2}}-\bar{u}_{1} u_{1} \bar{u}_{2} u_{2}- \\
& -3 r^{2} \bar{u}_{2} u_{2}+3 u_{2} r \bar{u}_{1} \bar{u}_{0}-2 \bar{u}_{2} u_{2} \bar{u}_{0} u_{0}-\bar{u}_{1} r^{2} u_{1}+3 u_{0} r \bar{u}_{2} u_{1},
\end{aligned}
$$

а также "комплексно-сопряженные" выражения для коэффициентов $\bar{a}, \bar{b}, \bar{c}$ и $\bar{q}$, где $u_{k}$ следует заменить на $\bar{u}_{k}$ и наоборот. Разрешая уравнения (П.1), мы получаем явное описание рационального вырождения кривой (5.10) в терминах коэффициентов конформного отображения (5.35). Однако в общем случае они могут быть определены только неявным образом через параметры потенциала $V(z, \bar{z})$. Простейший пример такого вырождения может быть описан формулой

$$
z^{2} \bar{z}^{2}-\frac{1}{3 \bar{t}} z^{3}-\frac{1}{3 t} \bar{z}^{3}+\left(t_{0}+\frac{1}{9 t \bar{t}}-18 t_{0}^{2} t \bar{t}\right) z \bar{z}+\left(3 t_{0}^{2}(1-3 t \bar{t})-27 t_{0}^{3} t \bar{t}-\frac{t_{0}}{9 t \bar{t}}\right)=0
$$

и это уравнение может быть разрешено с помощью конформного отображения

$$
z=\sqrt{t_{0}} w+\frac{3 t_{0} \bar{t}}{w^{2}}, \quad \bar{z}=\frac{\sqrt{t_{0}}}{w}+3 t_{0} t w^{2} .
$$

\section{ПРИЛОЖКНИЕ 2}

\section{Координаты в многосвязном случае}

Существование хорошо определенного двойственного базиса дифференциалов (6.37), удовлетворяющих условию ортонормированности (6.36), является ключевым свойством базиса функций $\mathrm{a}_{k}(6.34)$, вследствие которого моменты $\tau_{k}(6.33)$ являются "хорошими" локальными координатами, в отличие от $M_{n, \alpha}$, поскольку для функций $\left(z-z_{\alpha}\right)^{-n}$ невозможно подобрать дуальный базис.

Формулы суммирования

$$
\begin{array}{lll}
\frac{d z d \zeta}{\zeta-z}=\sum_{n=1}^{\infty} d \zeta \mathrm{a}_{n}(\zeta) d \mathrm{~b}_{n}(z), & \left|\prod_{\beta=0}^{g}\left(z-z_{\beta}\right)\right|<\left|\prod_{\beta=0}^{g}\left(\zeta-z_{\beta}\right)\right|, \\
\frac{d z d \zeta}{\zeta-z}=-\sum_{n=0}^{\infty} d \zeta \mathrm{a}_{-n}(\zeta) d \mathrm{~b}_{-n}(z), & \left|\prod_{\beta=0}^{g}\left(z-z_{\beta}\right)\right|>\left|\prod_{\beta=0}^{g}\left(\zeta-z_{\beta}\right)\right|,
\end{array}
$$

3 Теоретическая и математическая физика, т. 147, № 2, 2006 г. 
которые можно проверить непосредственно, позволяют повторить аргументы, изложенные в п. 6.1. Действительно, интеграл Коши (6.10), в котором интегрирование теперь проводится по всем граничным компонентам, определяет в каждой дырке $\mathrm{D}_{\alpha}$ аналитические дифференциалы $C^{\alpha}(z) d z$ (аналоги $C^{+}(z) d z$ из односвязного случая). $\mathrm{B}$ дополнительной области $\mathrm{D}^{\mathrm{c}}$ интеграл Коши по-прежнему определяет дифференциал $C^{-}(z) d z$, голоморфный везде в $\mathrm{D}^{\mathrm{c}}$, кроме бесконечности, где у него простой полюс. Разница между граничными значениями интегралов Коши равна $\bar{z}$ :

$$
C^{\alpha}(z)-C^{-}(z)=\bar{z}, \quad z \in \partial \mathrm{D}_{\alpha} .
$$

Из уравнения (6.11), которое можно написать отдельно для каждого контура, следует, что разница граничных значений производной интеграла Коши (6.10) $\left(\partial_{t} C^{\alpha}(\zeta)-\right.$ $\left.\partial_{t} C^{-}(\zeta)\right) d \zeta$ является для всех $\alpha$ чисто мнимым дифференциалом на границе $\partial \mathrm{D}_{\alpha}$. Разложение (П.5) ядра Коши гарантирует, что если $t$-деформация сохраняет все моменты $\tau_{k}, k \geqslant 0$, то дифференциал $\partial_{t} \bar{\zeta} d \zeta-\partial_{t} \zeta d \bar{\zeta}$ расширяется до голоморфного дифференциала на $\mathrm{D}^{\mathrm{c}}$.

Действительно, для значения $z$, лежащего достаточно близко к любой из точек $z_{\alpha}$, при любом $\alpha$ можно написать разложение $\partial_{t} C^{\alpha}(z)$ вида

$$
\partial_{t} C^{\alpha}(z) d z=\frac{1}{2 \pi i} \sum_{k=1}^{\infty} d \mathrm{~b}_{k} \partial_{t}\left(\oint_{\partial \mathrm{D}} \mathrm{a}_{k}(\zeta) \bar{\zeta} d \zeta\right)=\sum_{k=1}^{\infty} \partial_{t} \tau_{k} d \mathrm{~b}_{k}(z)
$$

и заметить, что оно тождественно равно нулю при $\partial_{t} \tau_{k}=0$. Поэтому $\partial_{t} C^{-}(z) d z$ представляет собой требуемое продолжение выражения $\partial_{t} \bar{\zeta} d \zeta-\partial_{t} \zeta d \bar{\zeta}$, у которого полюс в бесконечности исчезает благодаря условию $\partial_{t} \tau_{0}=0$.

Пользуясь принципом симметрии Шварца, заключаем, что $\partial_{t} C^{-}(z) d z$ продолжается до голоморфного дифференциала на дубле Шоттки. Если переменные $S_{\alpha}$ (6.35) сохраняются при $t$-деформации, то у этого голоморфного дифференциала обращаются в нуль все периоды по $B_{\alpha}$-циклам, а значит, он сам тождественно равен нулю. Это завершает доказательство утверждения, что любая деформация области, сохраняющая все $\tau_{k}$ и $S_{\alpha}$, тривиальна. В этом доказательстве переменные $S_{\alpha}$ были использованы только на последнем этапе, чтобы показать, что продолжение $\partial_{t} C^{-}(z) d z$ на дубль Шоттки $\Sigma$ как голоморфного дифференциала тривиально; вместо них можно аналогично использовать переменные $\phi_{\alpha}(6.32)$.

Покажем, что если эти переменные сохраняются при $t$-деформации, то $A_{\alpha}$-периоды продолжения $\partial_{t} C^{-}(z) d z$ равны нулю, а значит, это продолжение само тождественно равно нулю. Действительно, переменные $\phi_{\alpha}(6.32)$ можно представить в виде

$$
\phi_{\alpha}=-\frac{2}{\pi} \operatorname{Re} \int_{0}^{z_{\alpha}} d z \int_{\mathrm{D}^{\mathrm{c}}} \frac{d^{2} \zeta}{z-\zeta} .
$$

Дифференциал $(d z / \pi) \int_{\mathrm{D}^{\mathrm{c}}} d^{2} \zeta /(z-\zeta)$ равен $C^{\alpha}(z) d z$ при $z \in \mathrm{D}_{\alpha}$ и $\left(\bar{z}+C^{-}(z)\right) d z$ для $z \in \mathrm{D}^{\mathrm{c}}$. Пусть $\xi_{0}, \xi_{\alpha}$ - точки, в которых путь интегрирования, следующий из точки $z=0$ в $z=z_{\alpha}$, пересекает граничные контуры $B_{0}, B_{\alpha}$. Тогда

$$
\phi_{\alpha}=-2 \operatorname{Re}\left(\int_{0}^{\xi_{0}} C^{0}(z) d z+\int_{\xi_{\alpha}}^{z_{\alpha}} C^{\alpha}(z) d z+\int_{\xi_{0}}^{\xi_{\alpha}}\left(\bar{z}+C^{-}(z)\right) d z\right) .
$$


Выше было показано, что если $t$-деформация сохраняет переменные $\tau_{k}$, то все производные $\partial_{t} C^{\alpha}(z) d z=0$. Поэтому равенство нулю производной по $t$ величин $\partial_{t} \phi_{\alpha}=0$ дает

$$
0=-\partial_{t} \phi_{\alpha}=2 \operatorname{Re} \int_{\xi_{0}}^{\xi_{\alpha}} \partial_{t} C^{-}(z) d z
$$

Правая часть этого равенства является буквально $A_{\alpha}$-периодом голоморфного продолжения дифференциала $\partial_{t} C^{-}(z) d z$.

Благодарности. Автор благодарен Д. Васильеву, А. Забродину, В. Казакову, И. Кричеверу, А. Лосеву, А. Миронову, А. Морозову, Н. Некрасову и Л. Чехову за сотрудничество и обсуждение различных вопросов, затронутых в данной статье. Работа была частично поддержана РФФИ (грант № 04-01-00642), Программой поддержки ведущих научных школ (грант № 1578.2003.2), проектом NWO 047.017.015, проектом ANR "Геометрия и интегрируемость в математической физике" и Фондом поддержки Российской науки.

\section{Список литературы}

[1] А. М. Поляков, Калибровочные поля и струны, ИТФ им. Л. Д. Ландау, Черноголовка, 1995.

[2] J. Polchinski, Phys. Rev. Lett., 75 (1995), 4724; hep-th/9510017; Progr. Theor. Phys. Suppl., 123 (1996), 9; hep-th/9511157; Rev. Mod. Phys., 68 (1996), 1245; hep-th/9607050; TASI lectures on D-branes, hep-th/9611050.

[3] J. Polchinski, String Theory, Cambridge Univ. Press, Cambridge, 1998.

[4] J. M. Maldacena, TASI 2003 lectures on AdS/CFT, hep-th/0309246.

[5] А. В. Маршаков, УФН, 172 (2002), 977; hep-th/0212114.

[6] G. 't Hooft, Nucl. Phys. B, 72 (1974), 461.

[7] E. Brézin, C. Itzykson, G. Parisi, J.-B. Zuber, Commun. Math. Phys., 59 (1978), 35; F. David, Nucl. Phys. B, 257 [FS14] (1985), 45; V. Kazakov, Phys. Lett. B, 150 (1985), 282.

[8] E. Witten, Commun. Math. Phys., 252 (2004), 189; hep-th/0312171; Adv. Theor. Math. Phys., 8 (2004), 779; hep-th/0403199; D. Polyakov, Phys. Lett. B, 611 (2005), $173 ;$ hep-th/0501219.

[9] A. Gerasimov, A. Marshakov, A. Mironov, A. Morozov, A. Orlov, Nucl. Phys. B, 357 (1991), 565.

[10] S. Kharchev, A. Marshakov, A. Mironov, A. Orlov, A. Zabrodin, Nucl. Phys. B, 366 (1991), 569.

[11] T. Miwa, Proc. Japan Acad. A, 58 (1982), 9.

[12] S. Kharchev, A. Marshakov, A. Mironov, A. Morozov, Nucl. Phys. B, 397 (1993), 339.

[13] S. Kharchev, A. Marshakov, A. Mironov, A. Morozov, A. Zabrodin, Phys. Lett. B, 275 (1992), 311; hep-th/9111037; Nucl. Phys. B, 380 (1992), 181; hep-th/9201013.

[14] М. Концевич, Функи. анализ и его прилож., 25 (1991), 50; M. Kontsevich, Commun. Math. Phys., 147 (1992), 1.

[15] M. Fukuma, H. Kawai, R. Nakayama, Int. J. Mod. Phys. A, 6 (1991), 1385; Commun. Math. Phys., 143 (1992), 371. 
[16] S. Kharchev, A. Marshakov, "Topological versus nontopological theories and $p-q$ duality in $c \leqslant 12$-d gravity models", String Theory, Quantum Gravity and the Unification of the Fundamental Interactions. Proc. of Intern. Workshop on String Theory, Quantum Gravity and the Unification of Fundamental Interactions (Rome, Italy, 1993), eds. M. Bianchi, F. Fucito, E. Marinari, A. Sagnotti, World Scientific, Singapore, 1993; hep-th/9210072; Int. J. Mod. Phys. A, 10 (1995), 1219; hep-th/9303100.

[17] A. A. Migdal, Phys. Rep., 102 (1983), 199.

[18] F. David, Phys. Lett. B, 302 (1993), 403; hep-th/9212106.

[19] G. Bonnet, F. David, B. Eynard, J. Phys. A, 33 (2000), 6739; cond-mat/0003324.

[20] R. Dijkgraaf, C. Vafa, Nucl. Phys. B, 644 (2002), 3; hep-th/0206255; Nucl. Phys. B, 644 (2002), 21; hep-th/0207106; A perturbative window into non-perturbative physics, hep-th/0208048.

[21] G. Felder, R. Riser, Nucl. Phys. B, 691 (2004), 251; hep-th/0401191.

[22] I. Krichever, Commun. Pure. Appl. Math., 47 (1992), 437; hep-th/9205110.

[23] J. Fay, Theta Functions on Riemann Surfaces, Lect. Notes Math. V. 352, Springer, Berlin, 1973.

[24] Б. де Вит, А. В. Маршаков, ТMФ, 129 (2001), 230; hep-th/0105289.

[25] L. Chekhov, A. Mironov, Phys. Lett. B, 552 (2003), 293; hep-th/0209085.

[26] L. Chekhov, A. Marshakov, A. Mironov, D. Vasiliev, Phys. Lett. B, 562 (2003), 323; hep-th/0301071.

[27] V. A. Kazakov, A. Marshakov, J. A. Minahan, K. Zarembo, JHEP, 05 (2004), 024; hep-th/0402207; A. В. Маршаков, ТMФ, 142 (2005), 265; hep-th/0406056.

[28] M. Mineev-Weinstein, P. Wiegmann, A. Zabrodin, Phys. Rev. Lett., 84 (2000), 5106; nlin.SI/0001007; I. K. Kostov, I. Krichever, M. Mineev-Weinstein, P. B. Wiegmann, A. Zabrodin, " $\tau$-function for analytic curves", Random Matrices and Their Applications (Based on Talks and Lectures from the Workshop, Berkeley, CA, USA, February 22-26, 1999), Math. Sci. Res. Inst. Publ. V. 40, eds. P. Bleher, A. Its, Cambridge Univ. Press, Cambridge, 2001, 285; hep-th/0005259; A. Zabrodin, Matrix models and growth processes: From viscous flows to the quantum Hall effect, hep-th/0412219.

[29] V. A. Kazakov, A. Marshakov, J. Phys. A, 36 (2003), 3107; hep-th/0211236.

[30] B. Eynard, JHEP, 0301 (2003), 051; hep-th/0210047.

[31] I. Krichever, A. Marshakov, A. Zabrodin, Commun. Math. Phys., 259 (2005), 1; hep-th/0309010.

[32] А. В. Маршаков, "Матричные модели, комплексная геометрия и интегрируемые системы. II", ТMФ, 147:3 (2006), 399; hep-th/0601214.

[33] A. Marshakov, P. Wiegmann, A. Zabrodin, Commun. Math. Phys., 227 (2002), 131; hep-th/0109048.

[34] L. A. Takhtajan, Lett. Math. Phys., 56 (2001), 181; math.QA/0102164.

Поступила в редакцию 9.Х.2005 г. 